\title{
Weak-lensing magnification as a probe for the dark Universe
}

\author{
Manuel García Fernández
}





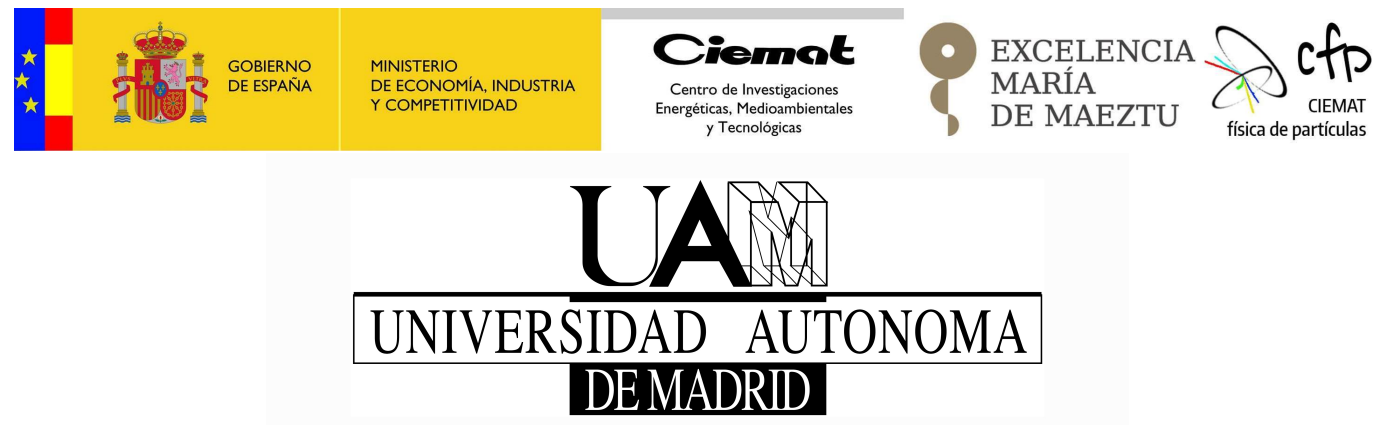

Departamento de Investigación Básica - División de Astrofísica de Partículas Centro de Investigaciones Energéticas Medioambientales y Tecnológicas

83

Departamento de Física Teórica - Facultad de Ciencias

Universidad Autónoma de Madrid

\section{Weak-lensing magnification as a probe for the dark Universe}

Tesis presentada por:

Thesis submitted by:

Manuel García Fernández

Para el grado de Doctor en Física Teórica.

For the degree of Ph.D. of Theoretical Physics.

Dirigido por:

Supervised by:

Dr. Eusebio Sánchez Álvaro

E3

Dr. Ignacio Sevilla Noarbe

Madrid, Marzo-Junio del 2017 

According to RD-99/2011, in partial fulfillment to obtain the 'Mención Internacional' qualification, this Thesis is written in English.

According to UAM's Normative (12/15/2011), Summary and Conclusions are also written in Spanish and included before the core of the Thesis, right after the table of contents.

Conforme al RD-99/2011, en requerimiento parcial para la obtención de la calificación de 'Mención Internacional', esta Tesis está redactada en Inglés.

Conforme al acuerdo del Consejo de Gobierno de la UAM (15/12/2011), el Resumen y las Conclusiones también se encuentran redactadas en Castellano y se incluyen antes del cuerpo de la Tesis justo después del índice.

This Thesis is part of the following publication archives:

The DES Collaboration archive: DES-2017-0265

Part of the results obtained during the development of this Thesis has been published under peer-review journals and proceedings. Their references are listed below.

Parte de los resultados obtenidos durante el desarrollo de esta Tesis han sido publicados en revistas y conferencias con un proceso de revisión por pares. Sus referencias bibliográficas se encuentran abajo.

M. Garcia-Fernandez, E. Sanchez and N. Sevilla-Noarbe. Magnification with wide-field photometric surveys. Highlights on Spanish Astrophysics XII. March 2017.

M. Garcia-Fernandez et al. Weak lensing magnification in the Dark Energy Survey Science Verification data, arXiv:1611.10326. November 2016. 

Hacemos una vasija con un pedazo de arcilla

y es el espacio vacío en el interior de la vasija el que la hace útil.

LAO TSÉ 



\section{Contents}

\section{Resumen y Conclusiones}

Resumen $\quad x i$

Conclusiones $\quad$ xii

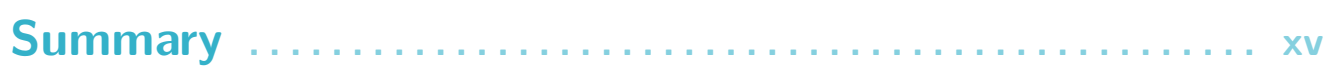

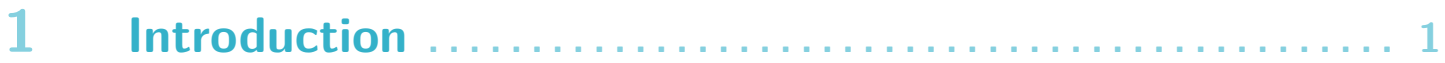

1.1 The $\Lambda$ CDM Cosmology 2

$\begin{array}{lll}1.2 & \text { The Cosmological Constant problem } & 7\end{array}$

$\begin{array}{lll}1.3 & \text { Theories for Dark Energy } & 7\end{array}$

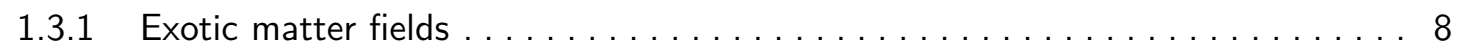

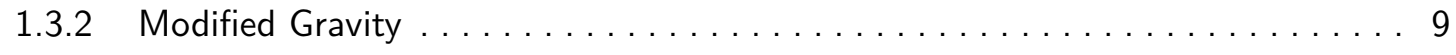

$\begin{array}{lll}1.4 & \text { Current status of dark energy constrains } & 10\end{array}$

1.5 Weak-lensing magnification as a probe for dark energy 12

2 Gravitational Lensing Theory ...................... 15

$\begin{array}{lll}2.1 & \text { Lens Equation on Gravitational Fields } & 17\end{array}$

2.2 Weak Gravitational Lensing $\quad 19$

2.2 .1 Magnification ............................. 20

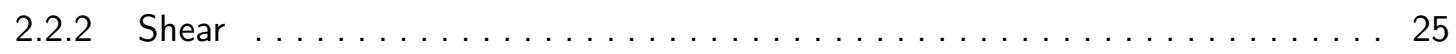

$\begin{array}{lll}2.3 & \text { Theoretical expressions for } \kappa(\theta) & 26\end{array}$ 
3 The Dark Energy Survey ...................... 29

3.1 The DECam 29

3.2 Survey strategy 30

3.3 The data reduction pipeline 33

3.4 Current status and latest results 33

4 Magnification in DES ............................ 39

4.1 Measuring Magnification through Number Count 39

4.2 Magnification in the MICE-GC simulation 42

4.3 Magnification in DES Science Verification data 44

4.3 .1 Data sample ................................... 44

4.3.2 Detection of the weak-lensing magnification signal $\ldots \ldots \ldots \ldots \ldots \ldots 47$

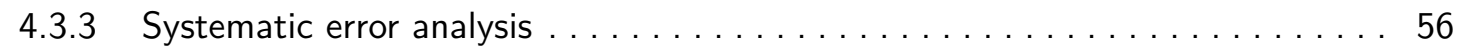

4.3 .4 Discussion ...................................... 67

4.4 Magnification in DES Year 1 data $\quad \mathbf{7 0}$

4.4 .1 Data sample ................................... 70

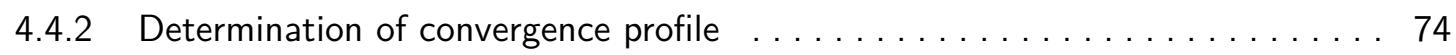

4.4 .3 Discussion ..................................... 81

5 Conclusions .................................. 83

Appendix Stellar contamination equation ...........8 85

Appendix Additional figures .................... 87

References ..................................... 103 


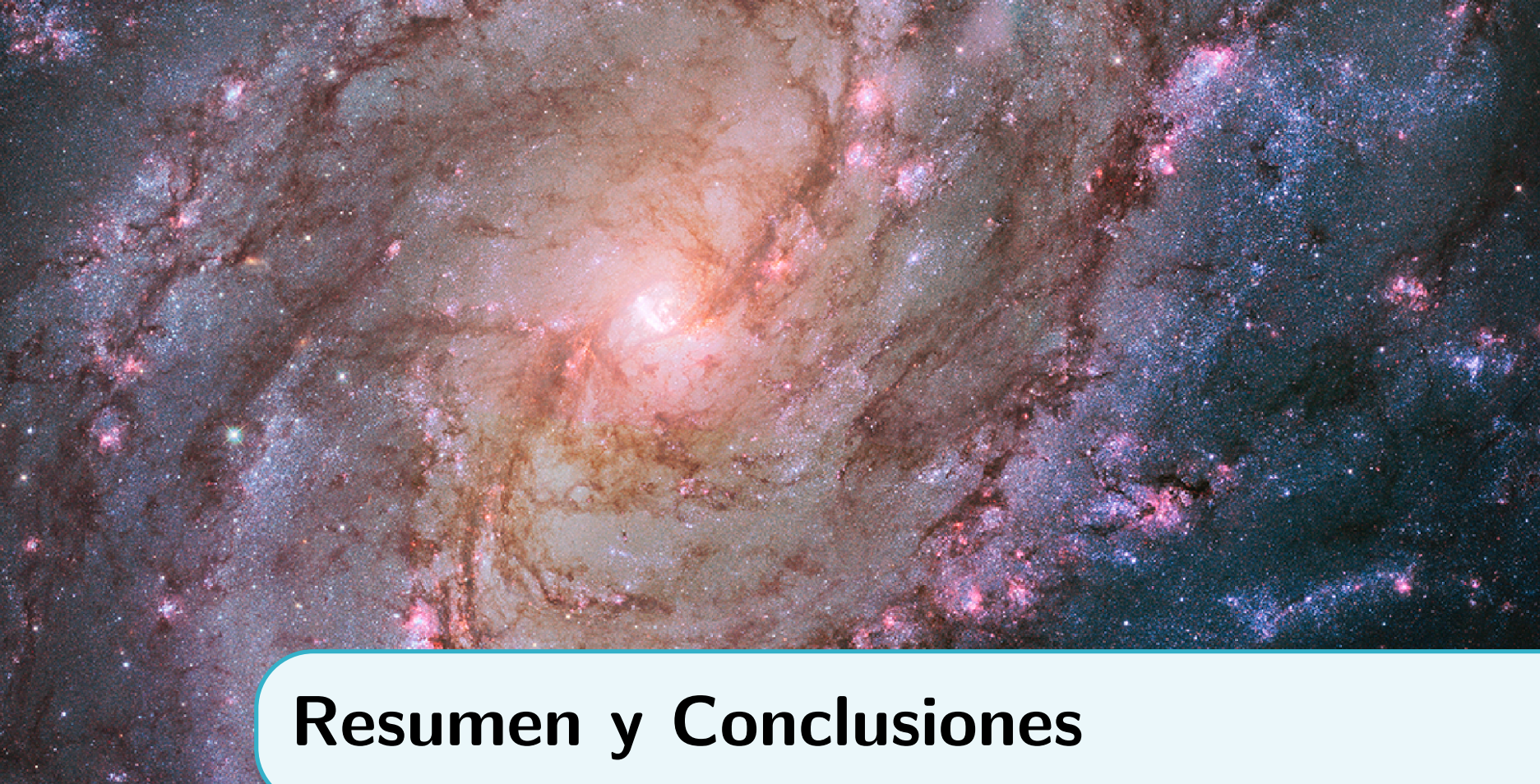

$+2$

\section{Resumen}

Las medidas cosmológicas muestran que la expansión del universo se está acelerando. Genéricamente, el agente causante de esta aceleración se llama energía oscura. Sin embargo, la naturaleza de la energía oscura constituye uno de los grandes misterios de la Física. Arrojar luz sobre la naturaleza de la energía oscura requiere la construcción de experimentos que cartografíen grandes volúmenes del universo. Uno de dichos experimentos es el Dark Energy Survey (DES) en el cual esta tesis ha sido desarrollada.

Entre las sondas observacionales que pueden desvelar la naturaleza de la energía oscura se encuentran las lentes gravitacionales débiles. El efecto lente gravitacional se produce al curvarse la trayectoria de los fotones por los campos gravitatorios, produciendo la deflexión de los rayos de luz. Entonces, la luz emitida por galaxias fuente lejanas es desviada por la materia localizada entre ellas y el observador. En el caso de fuentes extensas, a mayores del cambio en la posición, esto produce dos efectos observacionales: un aumento isótropo del tamaño (magnificación) y una elongación/contracción a lo largo de un eje (shear). Dado que el brillo superficial se conserva, el aumento isótropo del tamaño debido a la magnificación produce un incremento del flujo observado en las galaxias fuentes. Esto permite ver galaxias que estarían por debajo del umbral de detección si el efecto de lente gravitacional no existiese. Por tanto, cerca de las lentes la densidad de fuentes observada se incrementa. Este efecto se conoce como number-count magnification y permite medir el perfil de convergencia de la muestra seleccionada como lente, que es directamente dependiente del perfil de materia.

Esta tesis está dedicada al análisis de la magnificación por lentes gravitacionales débiles en el Dark Energy Survey. Se hacen dos análisis distintos en dos muestras de datos diferentes y con diferentes objetivos: el Science Verification (DES-SV) y el Year 1 (DES-Y1). El análisis realizado en DES-SV tiene como objetivo el 
desarrollo de técnicas para detectar la señal del efecto number-count magnification y la corrección de sus errores sistemáticos. El análisis de DES-Y1 emplea los métodos desarrollados en la muestra DES-SV para medir el perfil de convergencia de las regiones más vacías del universo -voids y troughs- para usarlas como una nueva sonda cosmológica.

\section{Conclusiones}

En esta tesis, el efecto de magnificación por lentes gravitacionales débiles ha sido medido usando dos muestras de datos del Dark Energy Survey: el Science Verification (DES-Y1) y el Year 1 (DES-Y1), con diferentes objetivos cada una. En el análisis realizado con el DES-SV se han desarrollado técnicas para medir el efecto y mitigar el impacto de posibles errores sistemáticos en la señal de number-count magnification con cartografiados fotométricos de gran campo. Por otro lado, el análisis del DES-Y1 ha hecho uso de las técnicas desarrolladas en el DES-SV para medir el perfil de convergencia de voids y troughs para proporcional medidas cosmológicas.

La naturaleza de la energía oscura -la responsable de la expansión acelerada del universo-, constituye uno de los grandes misterios de la cosmología moderna. Las regiones más vacías del cosmos -voids y troughs - están dominadas por la energía oscura, por lo que su estructura y evolución constituye un método muy potente para estudiar su naturaleza. Uno de los observables físicos de voids y troughs que es sensible a la energía oscura es su perfil de convergencia, que es una estimación directa del perfil de materia. Debido a la presencia de materia oscura, el perfil de materia es sólamente accesible a través de las lentes gravitacionales, dado que los otros métodos requieren de la parametrización de cómo las galaxias se relacionan con la materia oscura.

La magnificación por lentes gravitacionales proporciona una medida directa del perfil de convergencia. Hay tres observables que permiten determinar el perfil de convergencia: number-count magnification, la magnificación del tamaño y la magnificación del flujo/magnitud. Estos tres métodos pueden ser combinados para proporcionar medidas más precisas y mitigar los efectos de errores sistemáticos. Además, la magnificación puede ser combinada con medidas de gg-lensing para mejorar la correción de los errores sistemáticos dado que el gg-lensing es también sensible al perfil de convergencia, pero sus errores sistemáticos son distintos.

Entre los efectos sistemáticos más importantes a tener en cuenta se encuentran las condiciones de observación y el solapamiento por la determinación fotométrica del corrimiento al rojo de las galaxias. Para mitigar los errores inducidos por las condiciones de observación, se ha usado por primera vez la simulación de imágenes BALROG. El uso de BALROG ha demostrado proporcionar correciones fidedigas y no-sesgadas para este tipo de errores sistemáticos. Además, estas simulaciones permiten trazar las inhomogeneidades en la profundidad, permitiendo alcanzar la profundidad completa del experimento, aumentando así el número de galaxias disponibles. Por otro lado, el impacto del solapamiento por la determinación 
fotométrica del corrimiento al rojo ha sido estimado con ayuda de la simulación de N-cuerpos MICE, realizando luego cortes estrictos en el corrimiento al rojo, requiriendo un impacto despreciable en la señal de magnificación.

Finalmente, se ha medido por primera vez el perfil de convergencia de voids y troughs usando la magnificación. Esta medida abre una nueva ventana a una nueva clase de sondas cosmológicas, puesto que la magnificación producida por voids y troughs constituye una medida nueva e independiente para la energía oscura, que en un futuro inmediato puede proporcionar medidas cosmológicas competitivas y fidedignas. 



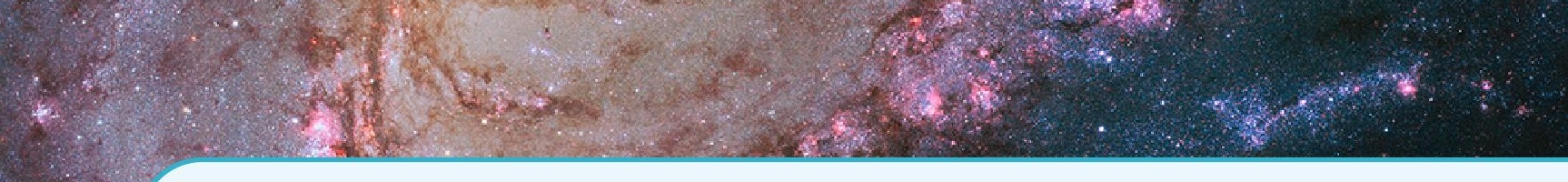

\section{Summary}

Cosmological measurements show that the expansion of the Universe is accelerating. Generically, the entity that causes this acceleration is called dark energy. Nevertheless, the nature of the dark energy constitutes one of the biggest puzzles in Physics. Shedding light on dark energy requires the construction of experiments able to survey large volumes of the Universe. One of those experiments is the Dark Energy Survey (DES) in which this thesis has been developed.

One of the observational probes that may unravel the nature of dark energy is the weak gravitational lensing. Weak-lensing is produced by the bending of the trajectory of photons by gravitational fields leading to the deflection of the light rays. Thus, the light emitted by distant galaxies is deflected by the matter located between them and the observer. For extended sources, in addition to the change in position, this leads to two observational effects: an isotropic size enlargement (magnification) and an elongation/shrink along one axis (shear). Since the surface brightness is preserved, the isotropic size enlargement due to magnification produces an increase on the observed flux of the background galaxies. This fact allows to see galaxies that would be beyond the detection threshold if gravitational lensing were not present. Thus, close to the lenses, the observed density of sources is increased. This effect is known as number-count magnification and allows to probe the convergence profile of the selected lens sample, that is a proxy for the matter profile.

This Thesis is devoted to the analysis of weak-lensing magnification on the Dark Energy Survey. Two analysis with different goals each are made on different data-sets: the Science Verification (DES-SV) and the Year 1 (DES-Y1). The DES-SV analysis aims the development of techniques to detect the weak-lensing number count magnification signal and the mitigation of systematic errors. The DES-Y1 analysis employs the methods used with the DES-SV data to measure the convergence profile of the emptiest regions of the Universe -voids and troughs- 
$x v i$

to use them as a new cosmological probe. 


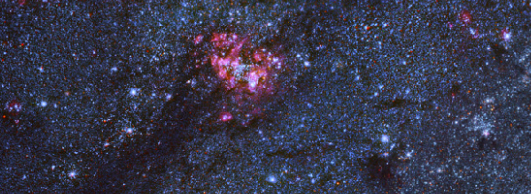

\section{Introduction}

Nature's change and evolution is a consequence of the dynamics that governs bodies and systems contained in the Universe. All the known interactions can be described in terms of the four Fundamental Forces: gravitation, weak, electromagnetic and strong in ascending order of relative strength. High Energy Physics was able to describe the weak, electromagnetic and strong forces in terms of a quantum field theory with $S U(3) \times S U(2) \times U(1)$ gauge symmetry group in what is known as the Standard Model [1-5]. Nevertheless, attempts to include gravitation in a similar frame have not provided satisfactory results yet.

The current consensus theory of gravitation is Einstein's General Relativity, that describes gravity as a deformation of space-time. It is based on two assumptions: physical laws must be the same in every coordinate system (Principle of Covariance) and Special Relativity must hold locally for every inertial observer (Principle of Equivalence). The most general second-order differential equation that holds these principles is the Einstein's field equation [6-9]

$$
R_{\mu \nu}-\frac{1}{2} R g_{\mu \nu}+\Lambda g_{\mu \nu}=\frac{8 \pi G_{N}}{c^{4}} T_{\mu \nu}
$$

where $R_{\mu \nu}, T_{\mu \nu}$ are the Ricci and energy-momentum tensor respectively, $c$ the speed of light and $R=g^{\mu \nu} R_{\mu \nu}$ is the Ricci scalar. The free parameters of this equation are $G_{N}$-Newton's constant- and $\Lambda$, the cosmological constant.

Previous equation can also be obtained from the variational formalism using the Einstein-Hilbert action [10]:

$$
S=\frac{c^{4}}{16 \pi G_{N}} \int d^{4} x \sqrt{-g}(R-2 \Lambda)+S_{M}
$$

with $S_{M}$ being the matter term of the action and $g=\operatorname{det}\left(g_{\mu \nu}\right)$. 


\subsection{The $\Lambda$ CDM Cosmology}

One of the consequences of Einstein's equation is that the metric tensor is not static, implying that the geometry of the Universe must evolve. Thus, space-time becomes a dynamical entity onto itself and its past and future evolution can be computed within the framework of General Relativity.

General Relativity is the first theory that allows to describe the Universe as a whole and by assuming that Earth is not in a special spot of the Universe -the Copernican Principle-, it follows that the Cosmos must be homogeneous and isotropic allowing to reach astonishing conclusions.

Assuming that the Universe is homogeneous and isotropic [11, 12], the only possible metric tensor is the Friedman-Lemaitre-Robertson-Walker (FLRW), given by the line element [13]

$$
d s^{2}=-d t^{2}+a^{2}(t)\left[\frac{d r^{2}}{1-K r^{2}}+r^{2}\left(d \theta^{2}+\sin ^{2} \theta d \phi^{2}\right)\right]
$$

where $a(t)$ is a function of time know as scale factor, $K=-1,0,1$ is the curvature of the universe (for an open, flat and closed Universe respectively) and $r, \theta, \phi$ are the spatial 3D spherical coordinates.

Solving Einstein's equation for this metric, an expression for the evolution of the scale factor with time can be obtained

$$
H^{2}(t) \equiv\left[\frac{\dot{a}(t)}{a(t)}\right]^{2}=\frac{8 \pi G_{N}}{3 c^{4}} \rho(t)-\frac{K}{a^{2}(t)},
$$

where the dot denotes time derivatives, and $\rho$ is the matter-energy density. The parameter $H$ has been defined as the expansion rate and its value at present $H_{0}$ is known as Hubble's constant.

The expansion rate can be expressed in terms of the normalized energy densities

$$
H^{2}(t)=H_{0}^{2}\left[\sum_{i} \Omega_{i}(t)-\Omega_{K}\right]
$$

with

$$
\Omega_{K} \equiv \frac{K}{\left[a(t) H_{0}\right]^{2}} \quad \text { and } \quad \Omega_{i}(t) \equiv \frac{8 \pi G_{N} \rho_{i}(t)}{3 H_{0}^{2}} .
$$

The parameter $\Omega_{i}$ is the density of the $i$-th matter/energy species whose evolution with time can be computed using Thermodynamics and assuming that each species behaves as a fluid with different equation of state. For non-relativistic matter -that is, matter with velocity $v \ll c^{-}$,

$$
\Omega_{M}(t)=\Omega_{M}^{0} a^{-3}(t),
$$

because $p \sim 0$, whereas for relativistic matter species -that is, $v \sim c^{-}$

$$
\Omega_{r}(t)=\Omega_{r}^{0} a^{-4}(t),
$$


because $p \sim \rho / 3$. Here $\Omega_{i}^{0}$ denotes the value on the present day of the $i$-th matter species and, by construction, the following equation holds:

$$
\sum_{i} \Omega_{i}^{0}=1+\Omega_{K}^{0}
$$

Taking into account that the matter species and the curvature evolve differently with time (Figure 1.1), its relative abundance at present fixes the expansion rate for the whole history of the Universe from birth to death.

General Relativity with the FLRW metric constitutes the theoretical basis for the current standard cosmological model: $\Lambda$ CDM. It states that the Universe is flat, homogeneous and isotropic. The observational basis of $\Lambda \mathrm{CDM}$ are:

- The dark matter: The presence of a new form of matter has been proposed to explain the measurement of the rotation curve of spiral galaxies [15-18]. Dark matter is a form of matter that is not in the Standard Model of High Energy Physics and interacts with the ordinary -a.k.a. baryonic- matter through gravity. Although several attempts have been made to constrain the nature of dark matter direct and indirectly, no evidence of its existence in laboratories has been found [19, 20]. On Figure 1.2, the determination of the energy density of dark energy and dark matter, assuming the $\Lambda$ CDM model can be seen. Rotation curves and the large-scale-structure of the Universe agree mutually on the existence of dark matter.

- The Cosmic Microwave Background (CMB): The Cosmic Microwave background is the oldest light of the Universe that can be observed. It is composed by the photons that decoupled from baryons at redshift $z=1100$, when the Universe became colder and the energy of the photons was not enough to ionize hydrogen. The energy distribution of the CMB-photons corresponds to a black-body spectrum with temperature $T_{C M B}=2.72 \mathrm{~K}$. The spatial distribution of the temperature anisotropies is related to the physics of the interaction of the photons and can be divided in two types: primary -those produced by the interaction of the photons with the baryons at the last scattering surface- and secondary -produced by the interaction of photons with the intergalactic medium and the gravitational potentials-. The power spectrum of the CMB anisotropies can be seen in Figure 1.3 with a fit to the $\Lambda$ CDM cosmology. This excellent agreement on such a very wide range of scales is a major success for $\Lambda$ CDM. In addition, the energy distribution of the CMB photons has a black-body spectrum, as predicted by $\Lambda$ CDM.

- The accelerated expansion of the Universe: The expansion of the Universe was discovered by Hubble [21]. Hubble established a linear relationship between the recession velocity of nearby galaxies which holds for low redshifts. Nevertheless, the improvement of the measurement with the inclusion of the data of the luminosity-distance relationship with type-Ia supernovae (SNIa) showed the accelerated expansion of the Universe [22] (Figure 1.4). The accelerated expansion of the Universe is translated on Einstein's field equation as a non-zero cosmological constant. 


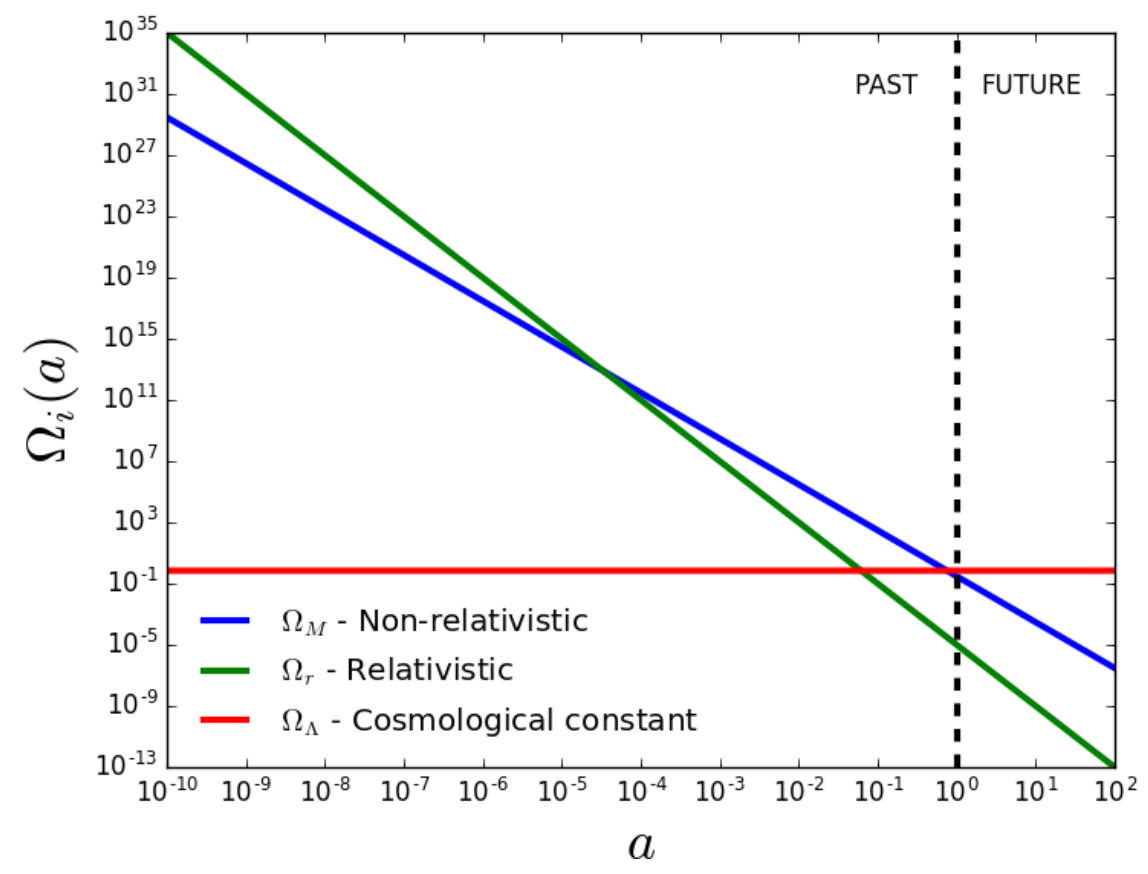

Figure 1.1: Energy density for different types of matter species as function of the scale parameter of the Universe: relativistic (radiation) on green, non-relativistic (matter) on blue, and cosmological constant on red. It can be seen that at present (black-dashed line), cosmological constant has just started to be dominant over the other species.

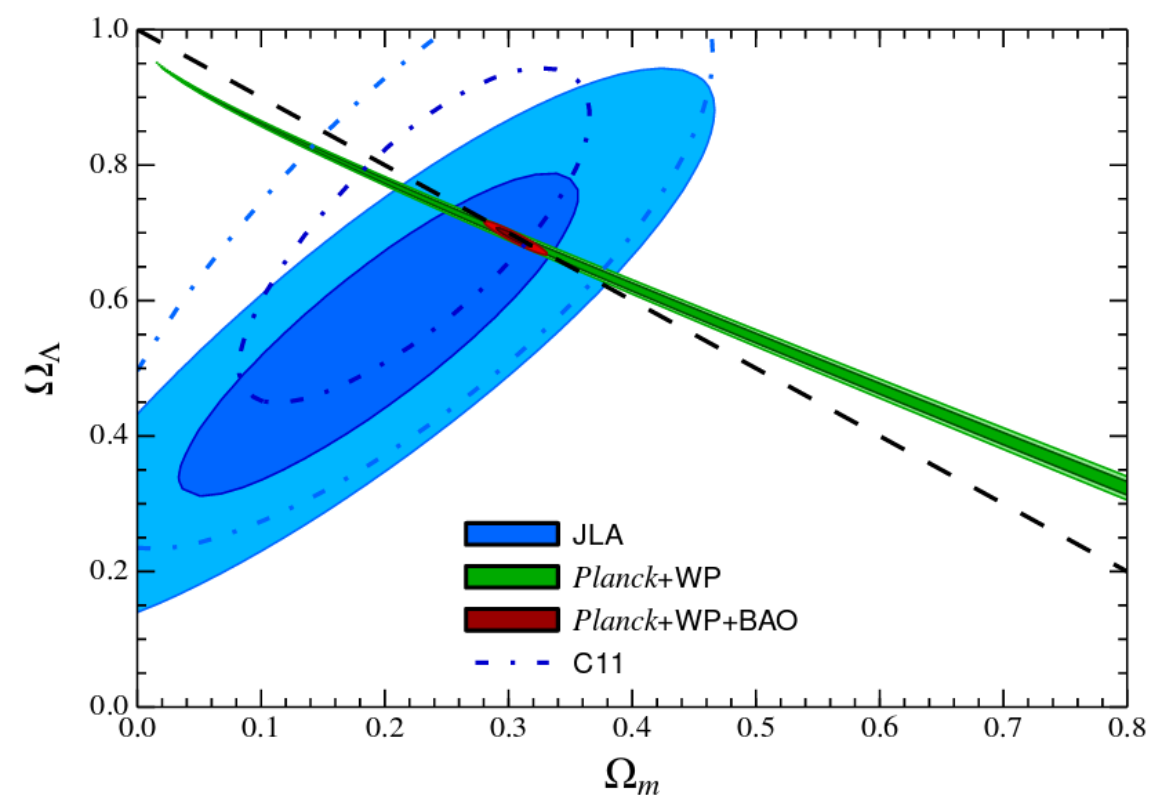

Figure 1.2: Determination of the non-relativistic matter and dark energy content of the universe with the combination of SNIa, BAO, CMB. Image credit: [14]. 


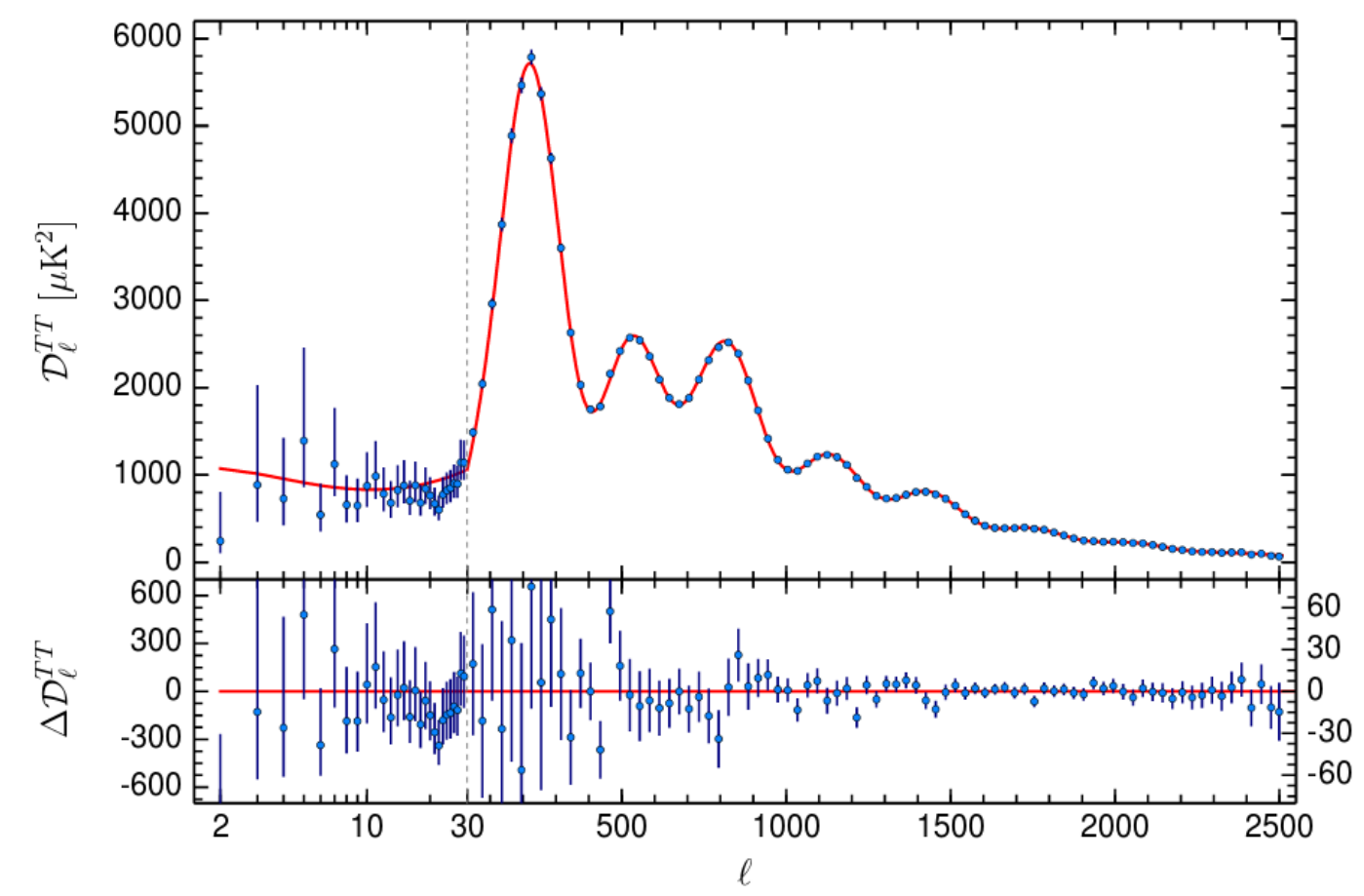

Figure 1.3: Power spectrum of the CMB. Red line is the best-fit to $\Lambda$ CDM. Image credit: [24]

- The baryon acoustic oscillations (BAO): The baryon acoustic oscillations are produced in the early Universe, when matter and radiation are coupled. Anisotropies in the gravitational fields lead to the collapse of matter on the wells of the potential. This leads to an increase of the temperature on the regions with higher density due to the matter-radiation coupling. The increase of the temperature produces a positive pressure that ejects matter outside the potential wells. The matter collapse and the radiation pressure produce acoustic waves that at matter-radiation decoupling leave a characteristic scale -the BAO scale-. This translates on peak on the angular distribution of matter and constitutes an standard ruler to measure cosmological distances (Figure 1.5).

- The primordial nucleosynthesis: The primordial nucleosynthesis or Big Bang nucleosynthesis is the production of the lightest elements of the periodic table other than hydrogen: deuterium, tritium, ${ }^{3} \mathrm{He},{ }^{4} \mathrm{He},{ }^{7} \mathrm{Li}$ and ${ }^{7} \mathrm{Be}$. The production of heavier elements other than the mentioned is not possible since there are not stable elements with mass-number five or eight. The relative abundance of these elements is governed -in addition to the nuclear properties of the elements- by the expansion of the Universe and the baryon-radiation ratio [23]. 


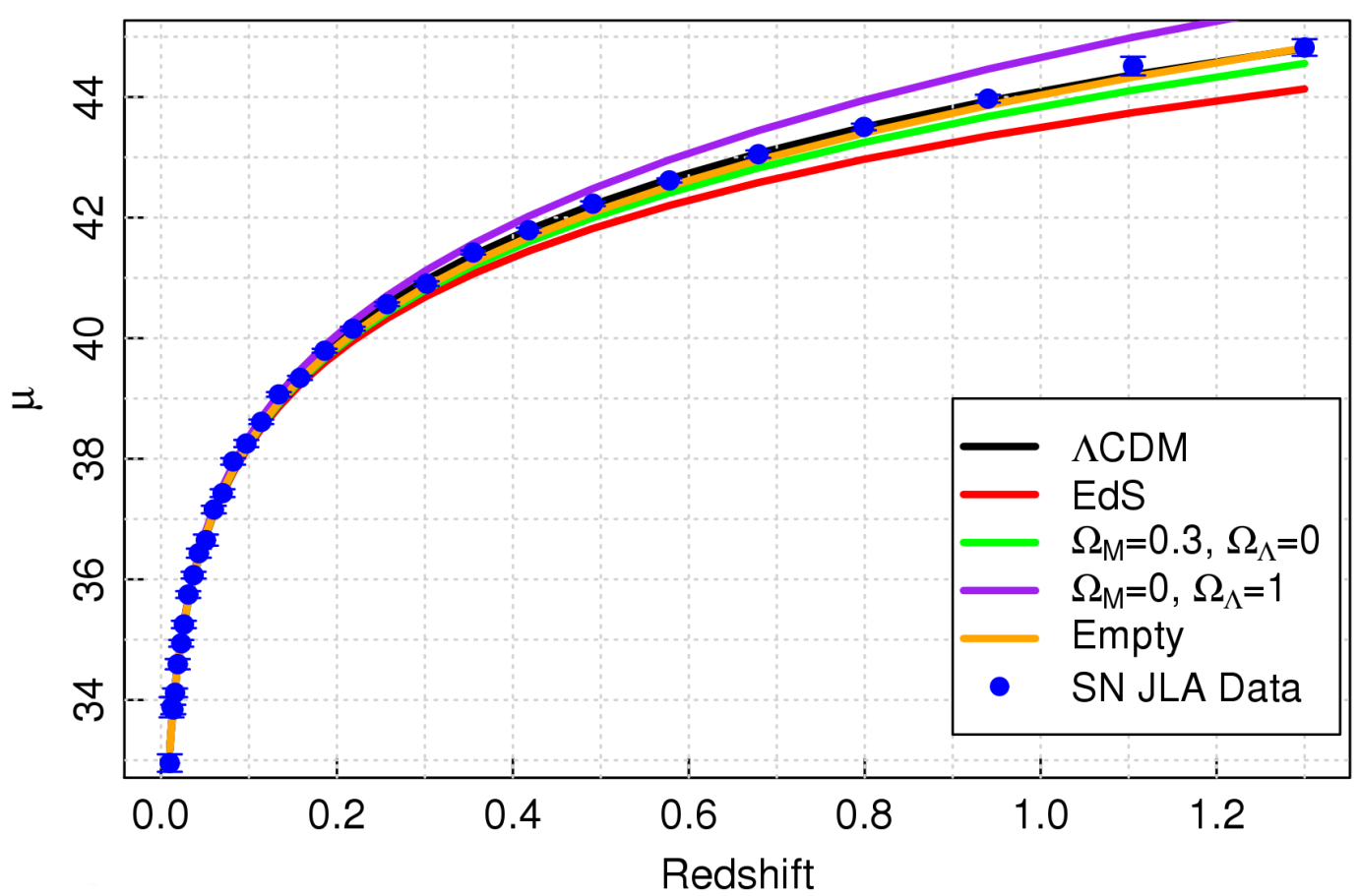

Figure 1.4: Distance modulus measured by the JLA supernovae. Results are compared with different cosmological models based on General Relativity.

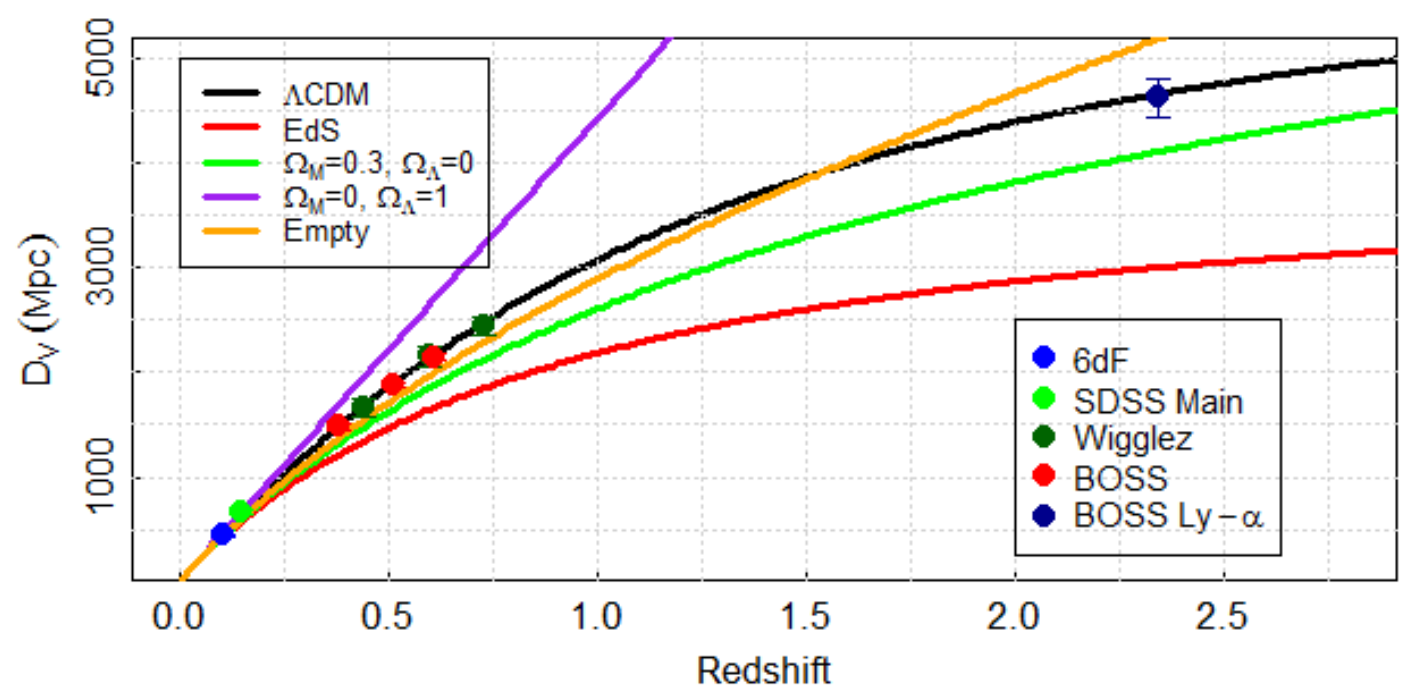

Figure 1.5: BAO distance-scale measured at different redshifts by different experiments. Results are compared with cosmological models based on General Relativity. 


\subsection{The Cosmological Constant problem}

The most general expression of Einstein's field equations contains the cosmological constant term, that can be absorbed on the right-hand-side of the Equation 1.1 and interpreted as a constant matter term known as dark energy:

$$
R_{\mu \nu}-\frac{1}{2} R g_{\mu \nu}=\frac{8 \pi G_{N}}{c^{4}} T_{\mu \nu}-\Lambda g_{\mu \nu}
$$

With this matter term, assuming $\Omega_{K}=0$, Equation 1.5 transforms into

$$
H^{2}(t)=H_{0}^{2}\left[\sum_{i} \Omega_{i}(t)+\Omega_{\Lambda}(t)\right]
$$

where $\Omega_{\Lambda}(t)=\Omega_{\Lambda}^{0}$ is the dark energy density. This new term is a time-independent constant that has the same value on every location of the Universe. Thus, while the other matter species have a density that decreases with time, the dark energy density is constant and becomes dominant at late cosmic times.

This new term may be regarded as a new matter species such that its energy density is given by

$$
\rho_{\Lambda}=\frac{3 H_{0}^{2}}{8 \pi G_{N}} \Omega_{\Lambda} \sim 10^{-47} \mathrm{GeV}^{4}
$$

If $T_{\mu \nu}=0$ there is no matter/energy, the $\Lambda g_{\mu \nu}$ term acts as an alternative source of energy and can be identified as the vacuum energy density [25-28]. The dominant source for the vacuum energy within the Standard Model of Particle Physics is the Higgs field [29, 30], a complex scalar-field that fills the Universe and gives mass to the elementary particles. The potential of the field is given by

$$
V(\phi)=\mu_{H}^{2} \phi^{\dagger} \phi+\frac{1}{4} \lambda_{H}\left(\phi^{\dagger} \phi\right)^{2}
$$

with $\mu_{H}$ the mass term, $\lambda_{H}$ the self-interaction of the field and $\phi, \phi^{\dagger}$ the Higgs field and its hermitian conjugate respectively. Thus, the cosmological constant can also be interpreted as the expected value of Higgs field [29, 30]

$$
\left\langle 0\left|\phi_{0}\right| 0\right\rangle=\frac{\left|\mu_{H}\right|}{\sqrt{\lambda_{H}}}=\sqrt{\frac{1}{\sqrt{2} G_{F}}}=246 \mathrm{GeV}^{4},
$$

which shows a huge discrepancy with the cosmological constant energy density as given by Equation 1.12. Here $G_{F}$ is the Fermi constant, that can be computed from the decay of the muon.

\subsection{Theories for Dark Energy}

As it has been stated previously, the observed value of the cosmological constant can not be explained with the Standard Model of High Energy Physics. Thus, one can try to explain accelerated expansion of the Universe with other class of theories. The simplest approach is to postulate the existence of new exotic fields with negative pressure that drive the accelerated expansion. The other possibility is an extension of General Relativity. 


\subsubsection{Exotic matter fields}

A possible explanation to the accelerated expansion of the Universe can be found on the presence on new quantum fields. The simplest case is known as quintessence and is defined as a scalar field $(\phi)$ that is added to the action defined at Equation 1.2 such that

$$
S=\frac{c^{4}}{16 \pi G_{N}} \int d^{4} x \sqrt{-g} R+S_{\phi}+S_{M}
$$

with

$$
S_{\phi}=\int d^{4} x\left[\frac{1}{2} g^{\mu \nu} \partial_{\mu} \phi \partial_{\nu} \phi-V(\phi)\right],
$$

where $V(\phi)$ is the potential of the field. On the FLRW metric, this leads to a substance with pressure and density respectively

$$
P_{\phi}=\frac{1}{2} \dot{\phi}^{2}-V(\phi) \quad \text { and } \quad \rho_{\phi}=\frac{1}{2} \dot{\phi}^{2}+V(\phi) .
$$

This can be parametrized as an ideal fluid with equation of state

$$
w_{D E}=\frac{P_{\phi}}{\rho_{\phi}}=\frac{\dot{\phi}^{2}-2 V(\phi)}{\dot{\phi}^{2}+2 V(\phi)} .
$$

Thus, it can be deduced that dark energy density evolves with the scale factor of the universe as

$$
\Omega_{\Lambda}(t)=\Omega_{\Lambda}^{0}[a(t)]^{-3\left(1+w_{\phi}\right)}
$$

if $w_{\phi}$ is constant.

This quintessence approach allows to explain any dark energy model by choosing properly the potential of the field. A detailed description of all the models can be found in [31]. The most widely used phenomenological description is made in terms of the equation of state of dark energy,

$$
P_{D E}=w_{D E} \rho_{D E}
$$

expanding the parameter $w_{D E}$ in a power series of the scale factor

$$
w_{D E}(t)=w_{0}+w_{a}[1-a(t)]
$$

where $w_{0}$ denotes the value of the equation of state parameter at present and $w_{a}$ its evolution -at first order- with time. The cosmological constant may be considered as an specific solution of this equation of state where

$$
w_{0}=-1 \quad \text { and } \quad w_{a}=0
$$

The key difference between the cosmological constant and other exotic forms of matter is that the latter provide a dark energy density that evolves throughout cosmic history. 


\subsubsection{Modified Gravity}

The other possibility to explain the accelerated expansion of the Universe is to assume that General Relativity is not valid at all cosmological distances and ages. Thus, data from cosmic expansion are not interpreted properly, requiring and extension of the theory. Extensions to General Relativity are known as modified gravity models.

In order to preserve the symmetries of General Relativity, the new gravitational field equations must be a function of the Ricci scalar. The most general class of those theories is known as $f(R)$ gravity [32, 33], and its approach is to let the action be a general function $(f)$ of the Ricci scalar:

$$
S=\frac{c^{4}}{16 \pi G_{N}} \int d^{4} x \sqrt{-g} f(R)+S_{M},
$$

that leads to the field equation

$$
R_{\mu \nu}-\frac{1}{2} R g_{\mu \nu}=\frac{8 \pi G_{N}}{c^{4}}\left(T_{\mu \nu}+T_{\mu \nu}^{R}\right)
$$

This field equation is similar to Equation 1.1 but has an additional term $T_{\mu \nu}^{R}$ that takes into account the additional curvature terms that can be modeled as a fluid on the same way as the energy-momentum tensor. The simplest case is $f(R)=R+\alpha R^{n}$ with $\alpha, n \in \mathbb{R}$, which has interesting cosmological solutions [34]. This theory has very distinctive phenomenology such as double Einstein rings with just one source galaxy on the strong lens regime [35] that -if found-, could be the smoking gun of these kind of theories. The existence of a double Einstein ring (SDSSJ0946+1006) has been reported [36], nevertheless it is a system with one lens and two sources at different redshifts, producing one ring of different diameter each.

More complicated models of modified gravity that may break the equivalence principle and the local Lorentz symmetry can be considered but are not going to be treated here. For a review the reference [37] can be consulted. The usual approach to explore the modifications to General Relativity [38] is to consider the departures of the observed metric respect to General Relativity. On the Newtonian gauge, the FLRW line element can be parametrized with the Newtonian and the lensing potential; $\Phi, \Psi$ respectively [38]:

$$
d s^{2}=a^{2}(\tau)\left[-(1+2 \Psi) d \tau^{2}+(1-2 \Phi) d x_{i} d x^{j}\right]
$$

with

$$
2 \nabla^{2} \Phi(a, k)=\frac{8 \pi G_{N}}{c^{2}} a^{2} \mu(a, k) \bar{\rho}_{M} \delta_{M}(a, k) \quad \text { and } \quad \gamma(a, k)=\frac{\Phi(a, k)}{\Psi(a, k)},
$$

where $\bar{\rho}_{M}$ is the average matter density, $\delta_{M}$ its fluctuations and $k$ is the wavenumber of the potentials. Here $\mu$ and $\gamma$ parametrize the departures from General Relativity, that is the specific case with

$$
\mu(a, k)=1 \quad \text { and } \quad \gamma(a, k)=1 .
$$




\subsection{Current status of dark energy constrains}

The nature of dark energy can be constrained using different cosmological probes [39] by measuring the $w_{0}, w_{a}$ parameters of its equation of state or the potentials $\Sigma, \gamma$ of modified gravity. There are two classes of cosmological probes: geometric and structure formation and evolution tests.

Geometrical measurements exploit the fact that the distances measured on cosmological scales depend on the metric tensor of the Universe, which is affected by its energy content and the assumed gravitational theory. These measurements include: the baryon acoustic oscillation peak-scale (BAO), the SNIa distanceladder, Alcock-Paczynski [40] tests and the integrated Sachs-Wolfe (ISW) effect. On the other side, structure evolution probes are based on the measurement of the inhomogeneities of the matter density field at different moments of cosmic history, which depend on the matter/dark-energy ratio and gravitational theory used. They include: the cluster-count evolution with redshift, redshift-space-distortions (RSD), lensing and the cosmic microwave background (CMB).

The combination of different cosmological probes leads to more accurate measurements and at the same time it breaks degeneracies on the determination of the cosmological parameters [39, 41].

The latest and more precise results constraining the cosmological parameters are provided by the Planck Collaboration 2015 results from the analysis of the Cosmic Microwave Background (CMB) [42], the recalibrated supernovae sample JLA [14], and the BAO scale-evolution with redshift by BOSS [43], WiggleZ [44], SDSS [45] and 6dF [46].

Dark Enery as a new quantum field, is determined by measuring the parameters of the equation of state (see Figure 1.6). Results are compatible with General Relativity plus cosmological constant, but the uncertainty on the parameters of the equation of state does not allow to exclude many models, since the current precision on the determination of the evolution parameter $w_{a}$ is still limited. Constrains on Modified Gravity models are also given in terms of the modified gravity potentials $\mu, \eta$ and $\Sigma$ (see Figure 1.7).

The latest results from the Sloan Digital Sky Survey (SDSS) and its upgrade BOSS, provide several measurements that show a good agreement with the $\Lambda$ CDM paradigm. These probes include: Alcock-Paczynski tests [47], the clustering of galaxies [48], baryon acoustic oscillations (BAO) [43, 49, 50] and redshift-space distortions [51].

Although several probes agree among them and seem to favour the cosmological constant as the origin of dark energy, there are some measurements that are in tension with Planck Collaboration 2015. Riess et al. latest direct determination of the Hubble constant using the cosmological distance ladder (parallax-cepheidsSNIa) [52], show a discrepancy at the $3 \sigma$ level with the Hubble constant measured 


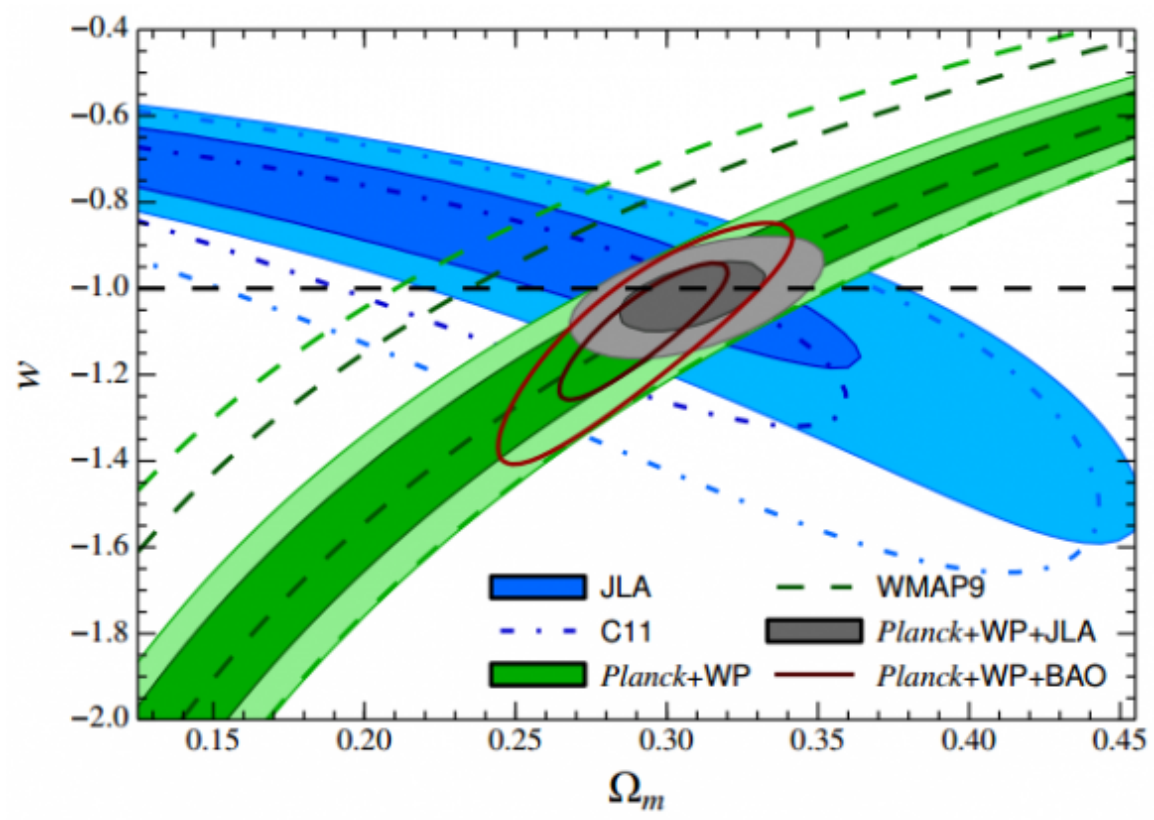

Figure 1.6: One- and two- sigma contours of the dark energy equation of state $w$ and matter content $\Omega_{M}$. Dashed line is General Relativity plus cosmological constant. Image credit: [14].

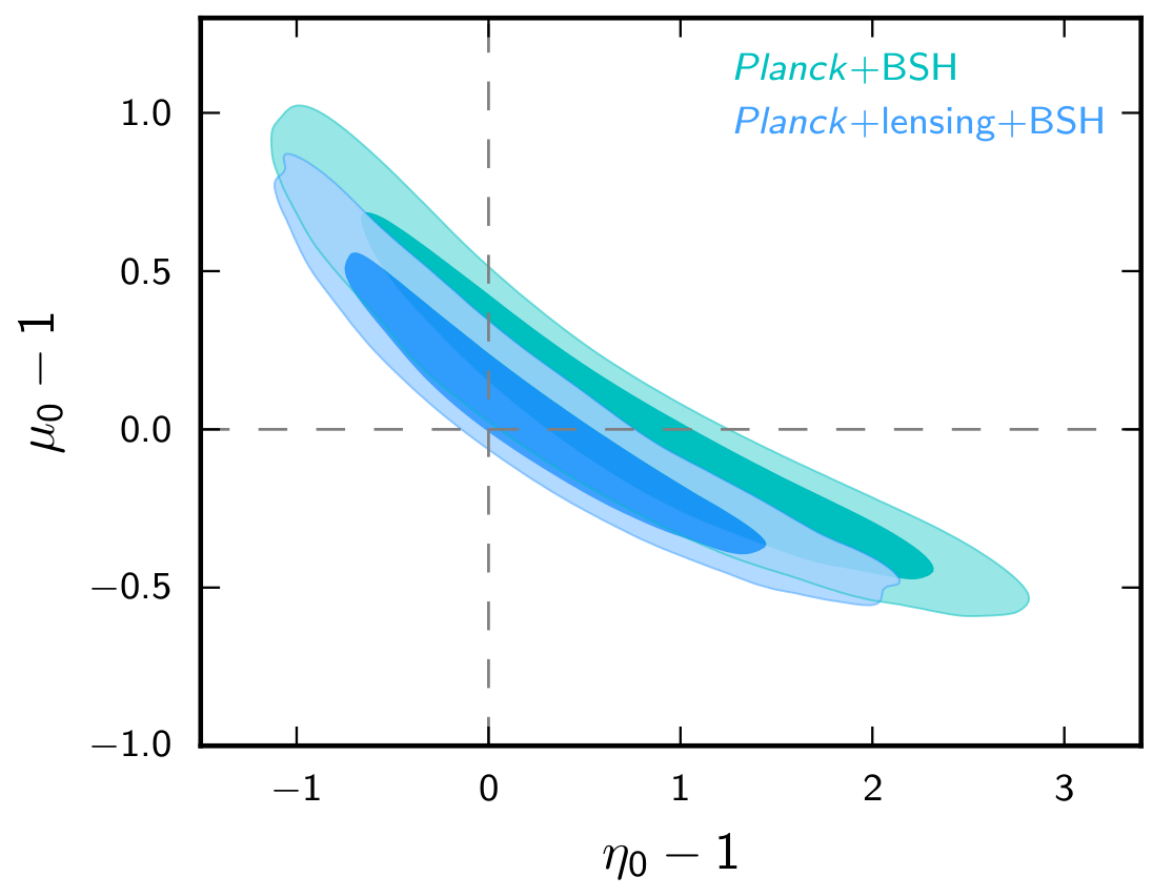

Figure 1.7: One- and two- sigma modified gravity potentials at present $\mu_{0}, \eta_{0}$. Dashed line is General Relativity plus cosmological constant. Image credit: [42]. 
by Planck Collaboration 2015. Weak-gravitational lensing by CFHTLens and KiDS show tension with Planck Collaboration CMB measurements if a General Relativity plus cosmological constant scenario is considered [53-55]. This tension may be alleviated if other models are considered, such as non-zero curvature and dark energy models [56-58]. Nevertheless, discrepancies can also be produced by systematic effects $[59,60]$. At any case, the weak-lensing/CMB discrepancy is still not significant enough to put the $\Lambda \mathrm{CDM}$ model in crisis.

The current cosmological model $-\Lambda \mathrm{CDM}-$, has demonstrated to be a solid theory that explains a wide range of physical effects and has passed the most stringent tests (Figure 1.3, Figure 1.4 and Figure 1.5). Although recent measurements show a small tension that may be alleviated in a non-cosmological constant scenario, caution is needed and special attention is required to do a proper systematic error estimation. This requires additional probes and redundant measurements of the same physical observable but with different sources of systematic errors. We have entered in the precision cosmology era.

\subsection{Weak-lensing magnification as a probe for dark energy}

Weak-lensing magnification is produced by the same physical entity as the gglensing, but has lower signal-to-noise (see chapter 2 for a full explanation of magnification and gg-lensing). Thus, the inclusion of magnification on a multiprobe analysis does not lead, generally, to a large improvement of the measurement. In order to achive this, we need to use magnification on environments and regimes that other probes can not reach.

The strength of magnification is that it allows to measure directly the matter profile of the large-scale-structures that conform the Universe. The whole matter structures of the Universe are only accessible directly trough gravitational lensing due to the presence of dark matter. Since dark matter is not visible and interacts only through gravity, assumptions on how the visible- -baryonic- - and darkmatter assemble together must be made on measurements other than gravitational lensing, introducing nuisance parameters.

A particular interesting system for weak-lensing measurements are voids. Since they have a lower matter content than the average Universe, their gravitational evolution is more dominated by dark energy. Thus, void properties depend on the nature of dark energy. If the abundance of large voids on the Universe is considered, it has been reported that its number increases in $f(R)$ gravity models [61, 62]. Nevertheless, if the shape of the void is measured, its ellipticity can be used as a probe for the parameters of the equation of state of dark energy [63-66], since the structure growth-factor on the line-of-sight has a variation due to the dark energy content, whereas on the transverse plane, growth-factor is constant. Finally, the radial distribution of matter around the center of a void -known as void profilehas demonstrated to be different on $f(R)$ theories and General Relativity [67-72]. Thus, by simply measuring the void matter profile, constrains on dark energy can 
be set. This implies that the direct determination of the matter profile of voids by weak-lensing magnification, constitutes a promising and independent new probe on dark energy.

This Thesis is devoted to the analysis of weak-lensing magnification using the Dark Energy Survey data. The analysis is carried out on two different data-sets of the mentioned experiment with two different goals each. The first analysis is carried out on the Science Verification data-set, aiming the detection of the magnification signal and the development of new techniques of systematic error mitigation. Once the magnification signal has been detected, a new analysis on the Year 1 data-release is made with the methodology that has been established previously. Year 1 analysis is qualitatively different since its goal is to measure the convergence profile of voids to use it as a probe for dark energy.

The next chapter (chapter 2), describes the general weak-lensing formalism and explains the magnification theory and its observational effects. The experiment where this Thesis has been developed - the Dark Energy Survey- is briefly described on chapter 3. The core of this work is found on chapter 4, where the analysis of the Science Verification data and the Year 1 data are described extensively, concluding on chapter 5 . 



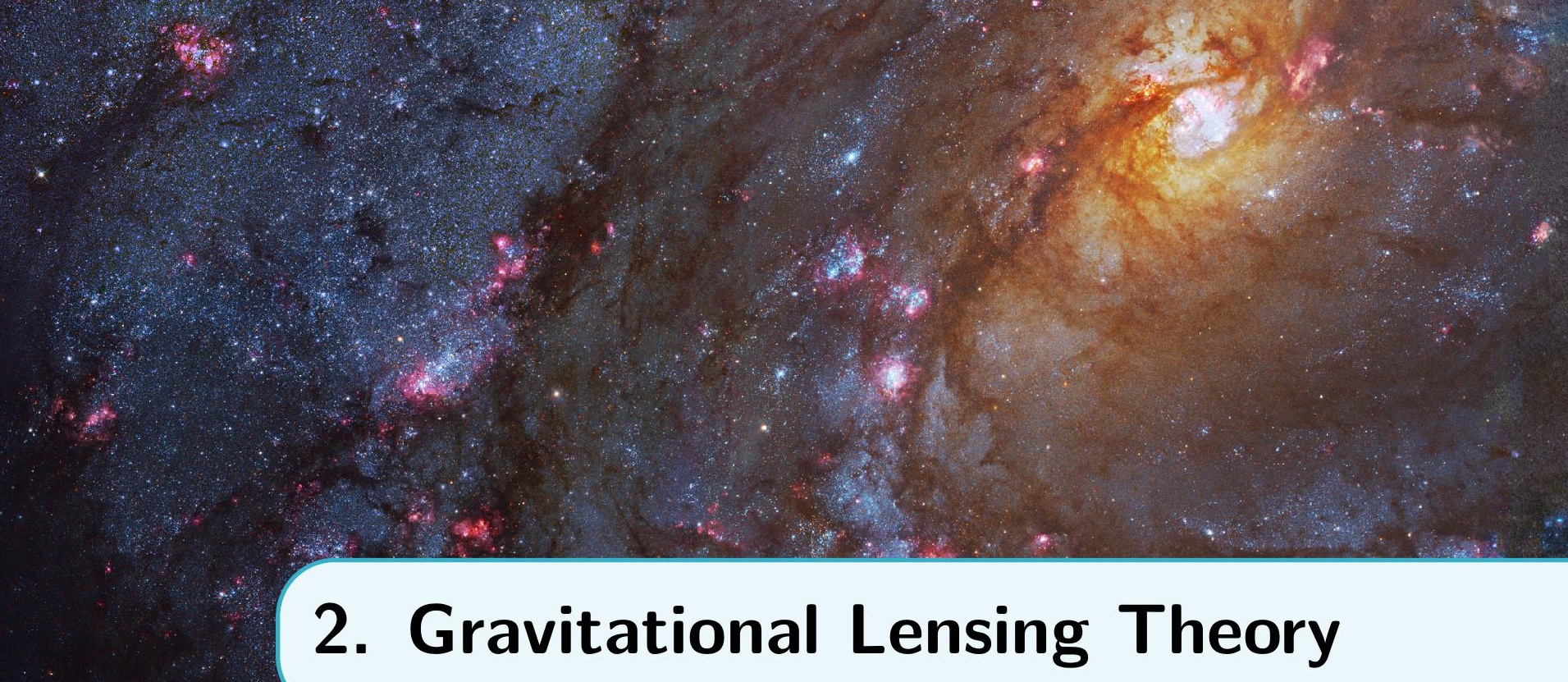

1.8.

\section{T.t.}

As it has been stated on chapter 1, the gravitational field acts on the energymomentum tensor. Thus, massless particles that are carriers of energy -such as photons- are also affected by gravity, leading to a bending of light rays. The first experimental determination of the gravitational light bending was performed by Dyson et al. in 1919 [73], four years after the publication of General Relativity. On this work, the observed apparent position of stars close to the Sun during a solar eclipse were measured and compared with their positions when the Sun is not in front of them. The positions of the stars were shifted as predicted by General Relativity.

Dyson et al. measurement of the gravitational deflection of the light emitted by a background object -a.k.a. source-, relied on the fact that the object that causes of the light deflection -a.k.a. the lens-, can be removed by its own seasonal motion. Nevertheless, this limits the measurement of gravitational light deflection to objects within the Milky Way. The study of the large-scale-structure of the Universe requires the use of extragalactic objects, implying that the object that acts as lens can not be removed, complicating the measurement.

One specific case of the gravitational light deflection happens when the observer, lens and source are aligned. This problem has cylindrical symmetry and leads to a very specific solution: the Einstein ring (Figure 2.1) [74]. On this configuration, the image of the background galaxy is distorted forming a ring around the lens galaxy, that its located at its center. The size of the ring is determined only by the mass of the lens and the distances of the lens and the source:

$$
\theta_{E}=\sqrt{\frac{4 G_{N} M}{c^{2}} \frac{d_{L S}}{d_{L} d_{S}}},
$$

where $G_{N}$ is Newton's gravitational constant, $M$ the mass of the lens, $d_{L S}$ the lens-source angular diameter distance and $d_{L}, d_{S}$ are the angular diameter distance 


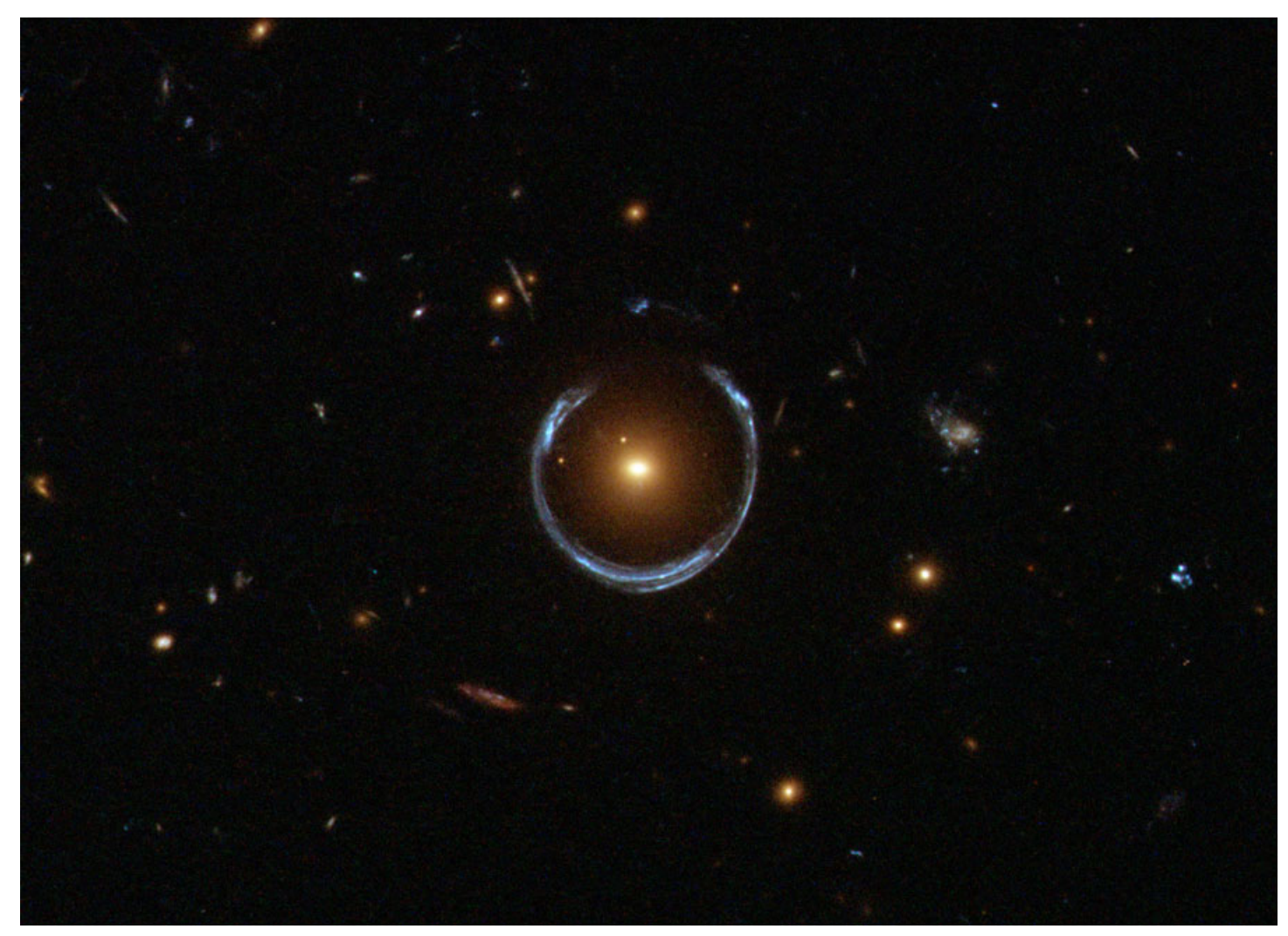

Figure 2.1: Image form the Hubble's Wide Field Camera 3 showing an Einstein ring. Central galaxy is the luminous red galaxy LRG-3-757. The blue annulus is a distant galaxy located behind the LRG. Image credit: NASA. 
to the lens and the source respectively.

Finding Einstein rings may be a product of serendipity or digging hard on wide-field images [75]. Nevertheless, the probability of finding a system where observer-lens-source are aligned is very small and only a modest number of Einstein rings are known ( $\sim 20$ on the Dark Energy Survey Science Verification data). A more general solution, where the system is not aligned can be found with the gravitational lens equation and is more useful for cosmology since the statistics grows enormously.

\subsection{Lens Equation on Gravitational Fields}

Since all the photons emitted by the source are bent coherently by the lens, the axis observer-lens constitute an optical convergent system. Thus a lens equation can be deduced using Geometrical Optics with the deflection angle $(\hat{\alpha})$ of a light ray - photon trajectory- given by General Relativity [39, 76-81]:

$$
\hat{\alpha}=\frac{4 G_{N} M}{\xi c^{2}} .
$$

Here $M$ is the mass of the point-particle (lens hereafter), $G_{N}$ is Newton's constant, $c$ the speed of light and $\xi$ the closest encounter distance (a.k.a. impact parameter). Using Figure 2.2 as reference and defining $\theta$ as the observed and $\beta$ the real lens-source angle, it can be deduced that

$$
\beta=\theta-\frac{D_{d s}}{D_{s}} \hat{\alpha}\left(D_{d} \theta\right)=\theta-\alpha(\theta)
$$

where $D_{d s}, D_{s}$ and $D_{d}$ are the source-lens, observer-source and observer-lens comoving distances.

Considering now an extended matter distribution, with density $\rho(\vec{r})$, where the observer is located at the origin. The position vector can be split such that

$$
\vec{r}=r_{\|} \hat{r}_{\|}+\vec{r}_{\perp}
$$

where $r_{\|} \hat{r}_{\|}$denotes the position on the direction defined by the axis observer-lens (line-of-sight or LoS hereafter) and $\vec{r}_{\perp}$ denotes a $2 \mathrm{D}$ vector on the plane transverse to the line-of-sight. Thus, the total matter distribution that the photon goes through from the source to the observer is given by

$$
\Sigma\left(\vec{r}_{\perp}, r_{\|}^{S}\right)=\int_{0}^{r_{\|}^{S}} d r_{\|} \rho\left(r_{\|}, \vec{r}_{\perp}\right),
$$

where $\vec{r}^{S}$ is the position of the source and the quantity $\Sigma$ is called the surface density. Taking into account the flat-sky approximation ${ }^{1}$-that is, all the transverse planes

\footnotetext{
${ }^{1}$ This approximation is responsible of part of the Planck-CFHTLens/KiDS tension.
} 


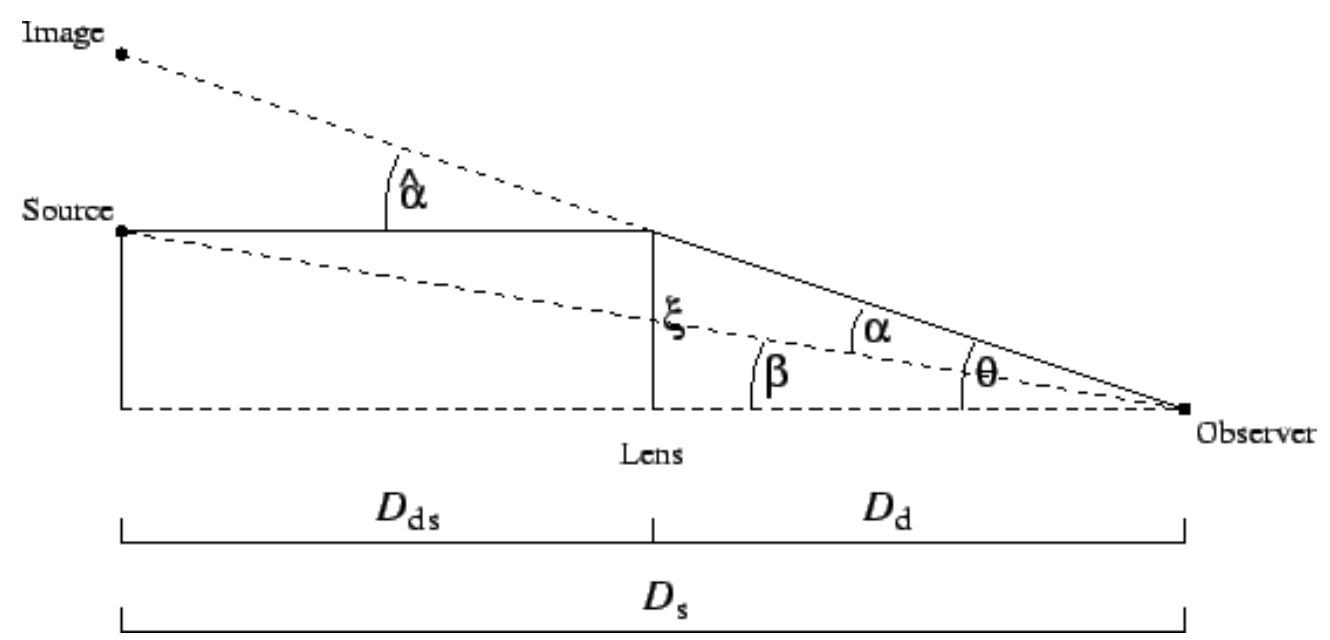

Figure 2.2: Optical system of the gravitational lensing caused by a point mass. Solid line is the actual photon trajectory. Dashed lines are the apparent trajectories with and without lensing. The distances $D_{s}, D_{d}, D_{d s}$ are expressed in comoving coordinates.

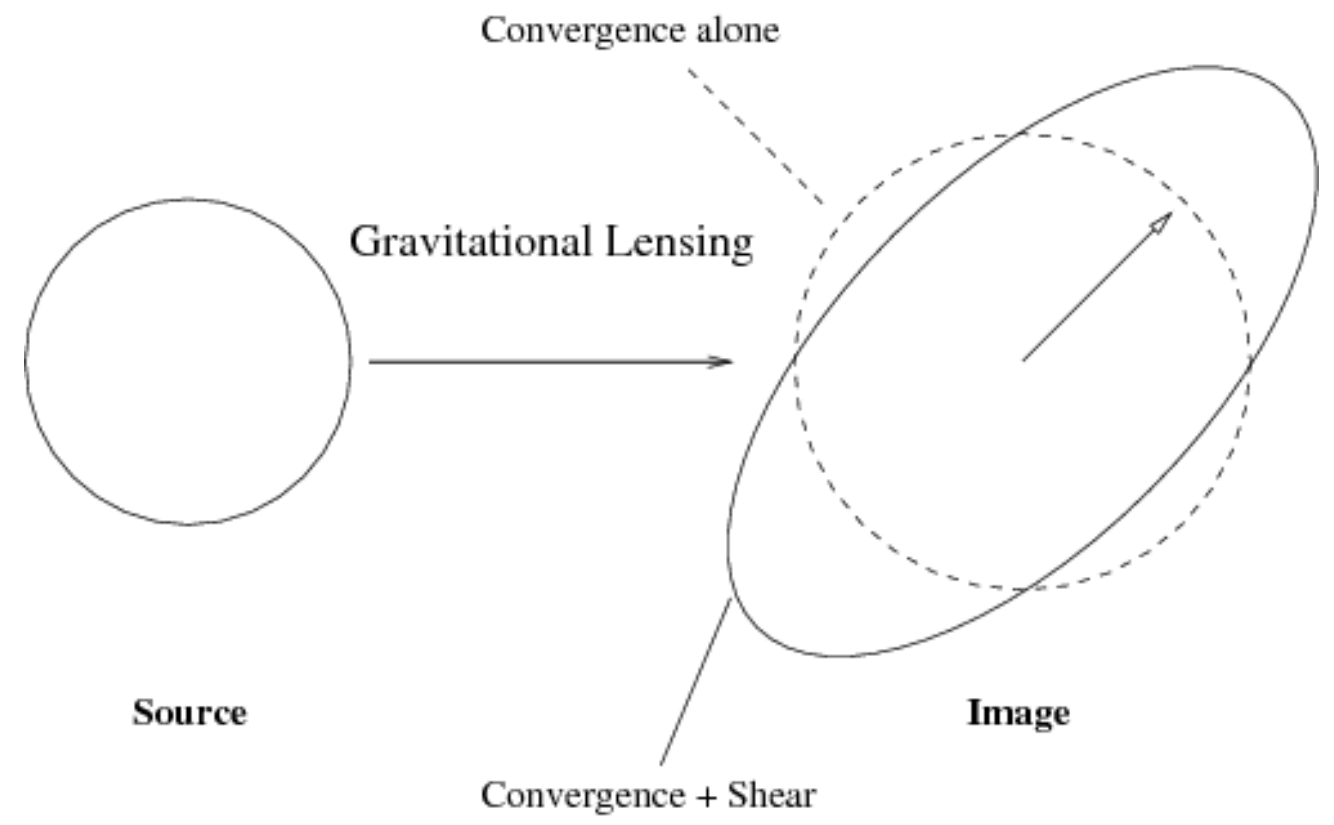

Figure 2.3: Weak-lensing distortion of an extended spherical object. Convergence leads to an isotropic enlargement, whereas shear produces an elongation/shrink along one axis. 
to LoS are parallel- and summing through all possible lens positions, Equation 2.2 becomes

$$
\hat{\vec{\alpha}}\left(\vec{r}_{\perp}, r_{\|}^{S}\right)=\frac{4 G_{N}}{c^{2}} \int d^{2} \vec{r}_{\perp} \Sigma\left(\vec{r}_{\perp}, r_{\|}^{S}\right) \frac{\vec{r}_{\perp}^{S}-\vec{r}_{\perp}}{\left|\vec{r}_{\perp}^{S}-\vec{r}_{\perp}\right|^{2}}
$$

This leads to a deflection angle

$$
\hat{\vec{\alpha}}\left(\vec{r}_{\perp}, r_{\|}^{S}\right)=\frac{1}{\pi} \int d^{2} \vec{r}_{\perp} \kappa\left(\vec{r}_{\perp}, r_{\|}^{S}\right) \frac{\vec{r}_{\perp}^{S}-\vec{r}_{\perp}}{\left|\vec{r}_{\perp}^{S}-\vec{r}_{\perp}\right|^{2}},
$$

where the convergence $(\kappa)$ and critical density $\left(\Sigma_{c}\right)$ have been defined such that

$$
\kappa\left(\vec{r}_{\perp}, r_{\|}^{S}\right)=\frac{\Sigma\left(\vec{r}_{\perp}, r_{\|}^{S}\right)}{\Sigma_{c}} \text { with } \Sigma_{c}=\frac{c^{2}}{4 \pi G_{N}} \frac{D_{s}}{D_{d} D_{d s}} .
$$

Defining the lensing potential as

$$
\psi\left(\vec{r}_{\perp}, r_{\|}^{S}\right)=\frac{1}{\pi} \int d^{2} \vec{r}_{\perp} \kappa\left(\vec{r}_{\perp}, r_{\|}^{S}\right) \ln \left|\vec{r}_{\perp}^{S}-\vec{r}_{\perp}\right|
$$

the deflection angle can be written as the gradient of the lensing potential on the transverse plane

$$
\hat{\vec{\alpha}}\left(\vec{r}_{\perp}, r_{\|}^{S}\right)=\nabla_{\perp} \psi\left(\vec{r}_{\perp}, r_{\|}^{S}\right)
$$

and the convergence as its laplacian,

$$
\kappa\left(\vec{r}_{\perp}, r_{\|}^{S}\right)=\frac{1}{2} \nabla_{\perp}^{2} \psi\left(\vec{r}_{\perp}, r_{\|}^{S}\right) .
$$

Thus, the lens equation from Equation 2.3 results

$$
\vec{\beta}=\vec{\theta}-\nabla_{\perp} \psi\left(\vec{r}_{\perp}, r_{\|}^{S}\right) .
$$

Taking into account the definition of the lensing potential, it can also be written in terms of the Newtonian gravitational potential $(\Phi)$ :

$$
\psi\left(\vec{r}_{\perp}, r_{\|}^{S}\right)=\frac{D_{d s}}{D_{s} D_{d}} \frac{2}{c^{2}} \int d r_{\|}^{S} \Phi\left(D_{d} \vec{r}_{\perp}, r_{\|}^{S}\right)
$$

Thus, gravitational lensing is a direct probe for the underlying gravitational field

As weak-lensing wide-field surveys become larger and more precise, the limit of validity of the flat-sky approximation is being reached [82, 83]. A solution without the flat-sky approximation can be found at Kitching et al. [84], and adds an additional prefactor that is function of the wavenumber on the power spectrum.

\subsection{Weak Gravitational Lensing}

In addition to the change in the observed position of the source, considering no absorption nor emission of photons between the source and the observer, Liouville's theorem implies that the surface brightness of the source $\left(I_{S}\right)$ is conserved,

$$
I_{S}\left(\vec{r}_{\perp}\right)=I_{S}\left[\vec{\beta}\left(\vec{r}_{\perp}\right)\right]
$$


Considering the weak-field regime, the lensing map can be linearized such that

$$
I_{S}\left(\vec{r}_{\perp}\right)=I_{S}\left[\vec{\beta}_{0}+\mathcal{J}\left(\vec{r}_{\perp 0}\right)\left(\vec{r}_{\perp}-\vec{r}_{\perp 0}\right)\right],
$$

where $\mathcal{J}\left(\vec{r}_{\perp}\right)$ is the jacobian matrix. By the integration-by-substitution theorem of calculus, the integral of the surface brightness at the lensed and un-lensed coordinate systems are related by

$$
\int I_{S}(\vec{\beta}) d \vec{\beta}=\operatorname{det}(\mathcal{J}) \int I_{S}\left[\vec{\beta}\left(\vec{r}_{\perp}\right)\right] d \vec{r}_{\perp}
$$

where $\operatorname{det}(\mathcal{J})$ denotes the determinant of the jacobian matrix. Thus, defining the luminosity of an extended object as the integral of its surface brightness, the luminosity for the cases with and without gravitational lensing $\left(L_{\mu}, L_{0}\right.$ respectively) are related by

$$
L_{\mu}=\frac{1}{\operatorname{det}(\mathcal{J})} L_{0}=\mu L_{0},
$$

where $\mu$ is called the magnification factor and is defined as the inverse of the determinant of the jacobian matrix of the lensing map.

Taking into account that the jacobian matrix is given by $\mathcal{J}=\left(\hat{n}_{\perp} \cdot \nabla_{\perp}\right) \vec{\beta}$ with $\hat{n}_{\perp}$ a unit vector on the plane transverse to LoS, by Equation 2.12 the jacobian can be expressed as

$$
\mathcal{J}=\left(\hat{n}_{\perp} \cdot \nabla_{\perp}\right) \vec{r}_{\perp}-\mathcal{J}=\left(\hat{n}_{\perp} \cdot \nabla_{\perp}\right) \nabla \psi
$$

resulting finally

$$
\mathcal{J}\left(\vec{r}_{\perp}\right)=\left(\delta_{i j}-\frac{\partial^{2} \psi}{\partial r_{i} \partial r_{j}}\right)=\left(\begin{array}{cc}
1-\kappa-\gamma_{1} & -\gamma_{2} \\
-\gamma_{2} & 1-\kappa+\gamma_{1}
\end{array}\right) .
$$

Here $\kappa$ is the convergence, an isotropic shape distortion and $\gamma_{1}, \gamma_{2}$ is the shear, an elongation/shrink on the shape along one of the axes (Figure 2.3).

Taking into account the Born approximation -that is, the light rays of a source galaxy are deflected by only one lens-, the derivatives of the previous equation can be evaluated on the unlensed coordinates.

\subsubsection{Magnification}

As it has been demonstrated in the previous section, gravitational lensing increases the observed luminosity on an extended object [85-89] such that $L_{\mu}=\mu L_{0}$ with

$$
\mu=\frac{1}{(1-\kappa)^{2}+\gamma_{1}^{2}+\gamma_{2}^{2}} \simeq 1+2 \kappa,
$$

where on the last step it has been used that $1 \gg \kappa \gg \gamma$. Taking into account the definition given in Equation 2.8, the convergence suffered by the photons emitted by a source located in sky direction $\hat{n}$ and redshift $z$ is given by

$$
\kappa(\hat{n}, z)=\frac{1}{2} \frac{\Sigma(\hat{n}, z)}{\Sigma_{c}} .
$$


Using the definition given in Equation 2.5 and the Poisson equation for the gravitational field this leads to

$$
\kappa(\hat{n}, z)=\int_{0}^{z} d z^{\prime} \frac{r\left(z^{\prime}\right)\left[r(z)-r\left(z^{\prime}\right)\right]}{r(z)} \nabla_{\perp} \Phi\left(\hat{n}, z^{\prime}\right),
$$

where $r(z)$ is the comoving distance at redshift $z$ and $\Phi$ the gravitational potential. Expressing now the gravitational potential as an homogeneus term plus a perturbation $\left(\Phi=\bar{\Phi}+\delta_{\Phi}\right)$, the previous equation can be expressed as a funcion of the matter density contrast $\left(\delta_{M}\right)$

$$
\nabla^{2} \Phi(\hat{n}, z)=\nabla^{2} \delta_{\Phi}(\hat{n}, z)=4 \pi G a^{2} \bar{\rho} \delta_{M}(\hat{n}, z)
$$

where $a=1 /(1+z)$ is the scale factor and $\bar{\rho}$ is the average density. This leads finally to $[90,91]$

$$
\kappa(\hat{n}, z)=\frac{3 H_{0} \Omega_{M}(z)}{2 c^{2}} \int_{0}^{z} d z^{\prime} \frac{r\left(z^{\prime}\right)\left[r(z)-r\left(z^{\prime}\right)\right]}{\left(1+z^{\prime}\right) r(z)} \delta_{M}(\hat{n}, z) .
$$

The convergence is the physical observable of magnification and it traces the matter density on the direction of line of sight whereas shear probes the matter on the transverse direction. This makes magnification and shear complementary measurements of the same phenomena. The dependence of magnification is usually split into two pieces: the lensing kernel,

$$
\mathcal{K}(z)=\frac{r\left(z^{\prime}\right)\left[r(z)-r\left(z^{\prime}\right)\right]}{\left(1+z^{\prime}\right) r(z)} .
$$

and the matter density contrast $\left(\delta_{M}\right)$. The lensing kernel contains only geometrical information and, for a given set of cosmological parameters, it is fixed. On the other side, the matter density contrast is sensitive to the growth of structure in the Universe. We see that weak lensing is both a geometrical and growth of structure probe of cosmology.

The convergence of the foreground sample can be probed tracing the three effects that it produces on the background sample:

- Change of the observed density: The increase of the observed luminosity of the galaxies allows to see sources that if there were no lensing, would be below our observational threshold nearby the location of the lenses. At the same time, an stretching of the solid angle behind the lenses causes a drop in the number density. This two effects compete between them and which one is over the other depends on the slope of the number counts of the sources. Thus, at the neighbourhood of the lenses, a change of the number density respect to the average is produced. This is known as number-count magnification.

- Shift on the observed magnitude: Since the increase of luminosity due to gravitational lensing is a short range effect, a shift on the observed magnitudes may be detected nearby the positions of the lenses. This requires, in principle, 
the knowledge of the unlensed magnitude of the sources. Nevertheless, although galaxies have a large variety of magnitudes, it can be assumed that, they are randomly distributed. Thus shifts on the magnitudes respect to the average can be detected.

- Size enlargement: All the effects above are a consequence of the conservation of surface brightness and the size enlargement of the sources. This enlargement can be statistically measured despite the fact that the unlensed size is not known. Nevertheless, since galaxies have a large variety of shapes and sizes that is strongly related to their evolution and age, no homogeneity assumption can be made and requires the use of empirical relations like the fundamental plane [92, 93].

Traditionally all these probes have been used independently, but the ideal scenario would be a three-way combination that may lead to a cancellation or better estimation of systematic errors.

As it has been mentioned before, the knowledge of the unlensed properties of the sources is physically impossible. Thus, all the observable quantities must be formulated in terms of changes of its variation respect the ensemble average with the distance to the lenses. The statistical way to do this is through the two-point angular correlation-function. This method provides a measurement of the average convergence profile of the lenses $(\kappa(\theta))$ as a function of its angular distance $\theta$ of a point to the lens.

\section{Estimation of $\kappa(\theta)$ with the number counts technique}

The two-point angular cross-correlation between positions of galaxies in the sample lens $(L)$ and in the source sample $(S)$ is defined as [94]

$$
\omega_{L S}(\theta)=\left\langle\delta_{O}\left(\hat{n}, z_{L}, f_{\mu}\right) \delta_{O}\left(\hat{n}^{\prime}, z_{S}, f_{\mu}\right)\right\rangle_{\theta} .
$$

Where $\delta_{O}\left(\hat{n}, z_{L}, f_{\mu}\right)$ is the observed galaxy density-contrast in the sky direction $\hat{n}$ and redshift $z_{L}$ with flux limit $f_{\mu}$. $\omega_{L S}$ is not zero, since due to magnification galaxies beyond the observable threshold will appear near the lenses introducing a non-uniform distribution of galaxies. Thus the observed galaxy density contrast can be expressed as

$$
\delta_{O}\left(\hat{n}, z, f_{\mu}\right)=\delta_{g}(\hat{n}, z)+\delta_{\mu}\left(\hat{n}, z, f_{\mu}\right),
$$

where $\delta_{g}$ is the intrinsic galaxy-density contrast (that is, without magnification) and $\delta_{\mu}$ is the density contrast due to magnification. Thus Equation 2.26 becomes

$$
\omega_{L S}(\theta)=\left\langle\delta_{g}\left(z_{L}\right) \delta_{g}\left(z_{S}\right)\right\rangle+\left\langle\delta_{g}\left(z_{L}\right) \delta_{\mu}\left(z_{S}\right)\right\rangle+\left\langle\delta_{\mu}\left(z_{L}\right) \delta_{g}\left(z_{S}\right)\right\rangle+\left\langle\delta_{\mu}\left(z_{L}\right) \delta_{\mu}\left(z_{S}\right)\right\rangle .
$$

Taking into account that $0<z_{L}<z_{S}$, and assuming that the lens and the source sample are well separated in redshift, the only non vanishing term is

$$
\omega_{L S}(\theta)=\left\langle\delta_{g}\left(\hat{n}, z_{L}\right) \delta_{\mu}\left(\hat{n}, z_{L}, f_{\mu}\right)\right\rangle_{\theta} .
$$

Let's define the magnification density contrast on the sky direction $\hat{n}$ as

$$
\delta_{\mu}\left(\hat{n}, z, f_{\mu}\right)=\frac{N_{\mu}\left(\hat{n}, z, f_{\mu}\right)}{N_{0}\left(\hat{n}, z, f_{0}\right)}-1
$$


where $N_{0}\left(\hat{n}, z, f_{0}\right)$ is the un-lensed cumulative number counts of sources located at redshift $z$, that is, the number of sources with observed flux greater than the threshold $f_{0}$. Conversely, $N_{\mu}\left(\hat{n}, z, f_{\mu}\right)$ is the lensed cumulative number counts affected by magnification.

Magnification by gravitational lenses increases the observed flux of background objects allowing to see fainter sources by an amount $f_{\mu}=f_{0} / \mu$. At the same time, it stretches the solid angle behind the lenses, reducing the surface density of sources an amount $N_{\mu}=N_{0} / \mu$, which translates into the density contrast as:

$$
\delta_{\mu}\left(\hat{n}, z, f_{\mu}\right)=\frac{N_{\mu}\left(\hat{n}, z, f_{\mu}\right)}{\mu N_{\mu}\left(\hat{n}, z, \mu f_{\mu}\right)}-1 .
$$

The cumulative number counts can be locally parametrized as a power law

$$
N_{\mu}\left(\hat{n}, z, f_{\mu}\right)=A\left(\frac{f_{\mu}}{f_{*}}\right)^{\alpha\left(f_{\mu}\right)},
$$

where $A, f_{*}$ are constant parameters and $\alpha\left(f_{\mu}\right)$ a function of the flux limit. Substituting this into Equation 2.31

$$
\delta_{\mu}\left(\hat{n}, z, f_{\mu}\right)=\mu^{\alpha\left(f_{\mu}\right)-1}-1 .
$$

Taking into account the weak-lensing approximation $\mu \simeq 1+2 \kappa$ along with Equation 2.20 and translating from fluxes to magnitudes this results

$$
\delta_{\mu}(\hat{n}, z, m)=2 \kappa(\hat{n}, z)[\alpha(m)-1]
$$

with

$$
\alpha(m)=2.5 \frac{d}{d m}\left[\log N_{\mu}(m)\right] .
$$

Thus Equation 2.26 becomes

$$
\omega_{L S}(\theta)=2[\alpha(m)-1]\left\langle\delta_{g}\left(\hat{n}, z_{L}\right) \kappa\left(\hat{n}^{\prime}, z_{S}\right)\right\rangle_{\theta}=[\alpha(m)-1] 2 \kappa(\theta),
$$

where $\kappa(\theta)$ is the convergence profile of the selected lenses.

\section{Estimation of $\kappa(\theta)$ with magnitude-shift magnification technique}

The magnitude-position-angular correlation function between the lens sample $(L)$ and the source sample $(S)$ is defined as

$$
\varphi_{L S}(\theta)=\left\langle\delta_{g}\left(z_{L}, \hat{n}\right) \delta_{m}\left(z_{S}, \hat{n}^{\prime}\right)\right\rangle_{\theta}
$$

Where, as on the last section $\delta_{g}(z, \hat{n})$ is the galaxy density contrast at redshift $z$ on the sky direction $\hat{n}$ and $\delta_{m}$ is the magnitude shift ${ }^{2}$, defined as

$$
\delta_{m}(z, \hat{n})=m_{\mu}(\hat{n}, z)-m_{0}(\hat{n}, z),
$$

\footnotetext{
${ }^{2}$ Do not confuse with $\delta_{M}$, the matter density contrast.
} 
where $m_{\mu}$ is the lensed magnitude and $m_{0}$ the unlensed magnitude. Taking into account that, as it has been demonstrated previously,

$$
f_{\mu}(\hat{n}, z)=\mu(\hat{n}, z) f_{0}(\hat{n}, z) \Leftrightarrow m_{\mu}(\hat{n}, z)-m_{0}(\hat{n}, z)=-2.5 \log \mu(\hat{n}, z),
$$

where on the last step it has been converted from fluxes to magnitudes. Thus, taking into account that

$$
\log (\mu) \simeq \log (1+2 \kappa) \simeq 2 \kappa
$$

the Equation 2.37 results finally

$$
\varphi_{L S}(\theta)=-5\left\langle\delta_{g}\left(\hat{n}, z_{L}\right) \kappa\left(\hat{n}^{\prime}, z_{S}\right)\right\rangle=-5 \kappa(\theta)
$$

where $\kappa(\theta)$ is the convergence profile of the lenses.

Nevertheless, reddening by the inter-galactic medium can also produce -unlike gravitational lensing- wavelength-dependent magnitude-shifts. Thus, the lensedplus-reddened fluxes are given by

$$
f_{\mu}\left(\hat{n}, z, \lambda_{\eta}\right)=\mu(\hat{n}, z) f_{0}(\hat{n}, z) e^{-\tau\left(\lambda_{\eta}\right)},
$$

that converted to magnitudes results in

$$
m_{\mu}\left(\hat{n}^{\prime}, z, \lambda_{\eta}\right)-m_{0}\left(\hat{n}^{\prime}, z, \lambda_{\eta}\right)=-2.5 \log \mu+\frac{2.5}{\ln 10} \tau\left(\lambda_{\eta}\right),
$$

where $\tau\left(\lambda_{\eta}\right)$ is the optical depth at the wavelength $\lambda_{\eta}$. The dust and the lensing components can be disentangled by defining the color-excess angular correlation function $\left(E^{\eta \nu}\right)$ between two-wavelengths $\lambda_{\eta}, \lambda_{\nu}$,

$$
E_{L S}^{\eta \nu}(\theta)=\left\langle\delta_{g}\left(\hat{n}, z_{L}\right)\left[m_{\mu}\left(\hat{n}^{\prime}, z_{S}, \lambda_{\eta}\right)-m_{\mu}\left(\hat{n}^{\prime}, z_{S}, \lambda_{\nu}\right)\right]\right\rangle_{\theta}
$$

Since gravitational lensing is achromatic, the only dependence with the wavelength comes from the extinction law

$$
E_{L S}^{\eta \nu}(\theta)=\frac{2.5}{\ln 10}\left\langle\delta_{g}\left(\hat{n}, z_{L}\right)\left[\tau\left(\hat{n}^{\prime}, z_{S}, \lambda_{\eta}\right)-\tau\left(\hat{n}^{\prime}, z_{S}, \lambda_{n} u\right)\right]\right\rangle
$$

Modeling the wavelength dependence of the optical depth as [95]

$$
\tau_{\eta}=\tau\left(\lambda_{\eta}\right)=\tau_{V}\left(\frac{\lambda_{V}}{\lambda_{\eta}}\right)^{\gamma},
$$

where $\tau_{V}$ is the optical depth at the $V$-band filter, $\lambda_{V}$ is the wavelength of the $V$-band filter and $\gamma \sim 1$ is a constant parameter. Thus, the color-excess crosscorrelation results finally

$$
E_{L S}^{\eta \nu}(\theta)=\lambda_{V}\left(\lambda_{\eta}^{-1}-\lambda_{\nu}^{-1}\right) \frac{2.5}{\ln 10}\left\langle\delta_{g}\left(\hat{n}, z_{L}\right) \tau_{V}\left(\hat{n}^{\prime}, z_{S}\right)\right\rangle
$$

At a wide field survey with several broad-band band-pass filters the scale dependence of the optical depth, $\tau_{V}\left(\hat{n}, z_{S}\right)$ can be constrained. 


\subsubsection{Shear}

From the Jacobian of the lensing map, it can be deduced that the transformation is not isotropic producing an elongation along one of the axis $\left(r_{1}, r_{2}\right)=\vec{r}_{\perp}$. Thus, an intrinsically round galaxy is seen as elliptical. On the case of elliptical galaxies, statistically they present a null global ellipticity ${ }^{3}$. From the definition of Equation 2.19, the shear components are given by:

$$
\gamma_{1}\left(\vec{r}_{\perp}\right)=-\frac{1}{2}\left(\frac{\partial^{2} \psi}{\partial r_{1}^{2}}-\frac{\partial^{2} \psi}{\partial r_{2}^{2}}\right) \text { and } \gamma_{1}\left(\vec{r}_{\perp}\right)=-\frac{\partial^{2} \psi}{\partial r_{1} \partial r_{2}} .
$$

The shear fields $\gamma_{1}, \gamma_{2}$ can be expressed as Fourier series such that:

$$
\tilde{\gamma}_{1,2}\left(\vec{k}_{\perp}\right)=\int \gamma_{1,2}\left(\vec{r}_{\perp}\right) e^{-i \vec{k}_{\perp} \cdot \vec{r}_{\perp}} d^{2} \vec{r}_{\perp}
$$

and

$$
\gamma_{1,2}\left(\vec{r}_{\perp}\right)=\frac{1}{(2 \pi)^{2}} \int \tilde{\gamma}_{1,2}\left(\vec{k}_{\perp}\right) e^{i \vec{k}_{\perp} \cdot \vec{r}_{\perp}} d^{2} \vec{k}_{\perp}
$$

Expressing the differential equation at the Fourier space with the usual approach $\partial / \partial r_{1} \rightarrow i r_{1}$ it leads to

$$
\gamma_{1}\left(\vec{k}_{\perp}\right)=\frac{1}{2}\left(r_{1}^{2}-r_{2}^{2}\right) \tilde{\psi}\left(\vec{k}_{\perp}\right) \text { and } \gamma_{2}\left(\vec{k}_{\perp}\right)=\frac{1}{2} r_{1} r_{2} \vec{\psi}\left(\vec{r}_{\perp}\right)
$$

Considering a plane-wave perturbation of the lensing potential, it is useful to align the axis of the perturbation with those of the shear field such that

$$
\tilde{\gamma}_{E}\left(\vec{k}_{\perp}\right)=\cos \left(2 \phi_{\vec{k}_{\perp}}\right) \tilde{\gamma}_{1}\left(\vec{k}_{\perp}\right)+\sin \left(2 \phi_{\vec{k}_{\perp}}\right) \tilde{\gamma}_{2}\left(\vec{k}_{\perp}\right)
$$

and

$$
\tilde{\gamma}_{B}\left(\vec{k}_{\perp}\right)=\cos \left(2 \phi_{\vec{k}_{\perp}}\right) \tilde{\gamma}_{1}\left(\vec{k}_{\perp}\right)-\sin \left(2 \phi_{\vec{k}_{\perp}}\right) \tilde{\gamma}_{2}\left(\vec{k}_{\perp}\right)
$$

Resulting finally that

$$
\tilde{\gamma}_{E}\left(\vec{k}_{\perp}\right)=\vec{k}_{\perp}^{2} \tilde{\psi}\left(\vec{k}_{\perp}\right) \text { and } \tilde{\gamma}_{B}\left(\tilde{k}_{\perp}\right)=0
$$

The fact that $\gamma_{B}$ is zero, constitutes a necessary (but not sufficient) proof for the lack of systematic effects on any shear measurement.

Reaching this point, two kinds of two-point statistics can be build: the pointshear $^{4}$ and the shear-shear two-point angular-correlation functions. From this two, we will only focus to gg-lensing due to its direct connection to magnification.

\section{The gg-lensing.}

Defining $\tilde{\epsilon}$ as the observed ellipticity, taking into account shear distortions it can be expressed as:

$$
\tilde{\epsilon}=\tilde{\epsilon}_{i}+\tilde{\gamma}
$$

\footnotetext{
${ }^{3}$ If they do not present null ellipticity it is a systematic effect known as intrinsic alignment.

${ }^{4}$ This is usually called galaxy-galaxy- (or gg-) lensing.
} 
where $\tilde{\epsilon}_{i}$ is the intrinsic ellipticity of the galaxy. As stated previously, without loss of generality, shear coordinates can be rotated. Thus, let define the tangential shear $\gamma_{t}$ as

$$
\gamma_{t}=-\left[\cos \left(2 \phi_{\vec{k}_{\perp}}\right) \tilde{\gamma}_{1}\left(\vec{k}_{\perp}\right)+\sin \left(2 \phi_{\vec{k}_{\perp}}\right)\right] \tilde{\gamma}_{2}\left(\vec{k}_{\perp}\right)=-\gamma_{E}
$$

On the new coordinates, this leads to

$$
\epsilon=\epsilon_{i}+\gamma_{E}
$$

where $\tilde{\epsilon}, \tilde{\epsilon}_{i}$ are the ellipticity on the new coordinates. Let $p(\epsilon)$ the distribution of ellipticity of the sources. At the small distortion regime, assuming that ellipticity is isotropic, it follows that

$$
p(\epsilon)=p\left(\epsilon_{i}\right)+\gamma_{t} \cos 2 \phi \frac{\partial p(\epsilon)}{\partial \epsilon} .
$$

Here $\phi_{\vec{k}_{\perp}}$ is the angle of orientation of the principal axis of the galaxy. Integrating over all the ellipticity, they can be translated to orientation angle,

$$
p(\phi)=\frac{2}{\pi}\left[1-\left\langle\gamma_{t}\right\rangle \cos 2 \phi\left\langle\frac{1}{\epsilon}\right\rangle\right]
$$

Thus, measuring the ellipticity -or orientation angles-, shear E-modes can be measured, probing directly the underlying lensing potential.

Tangential shear can be estimated to be

$$
\left\langle\gamma_{t}\right\rangle=-\frac{\Delta \Sigma}{\Sigma_{c}}
$$

with

$$
\Delta \Sigma=\bar{\Sigma}(\theta)-\Sigma(\theta)
$$

where $\bar{\Sigma}(\theta)$ denotes the average surface density on an disk of angular size $\theta$. Thus the tangential shear can be related to the convergence profile as

$$
\left\langle\gamma_{t}\right\rangle=-[\bar{\kappa}(\theta)-\kappa(\theta)]
$$

The last equation demonstrates that gg-lensing and magnification are produced by the same physical effect. Nevertheless, they have different systematic effects. This can be exploited in order to produce accurate and reliable cosmological measurements.

\subsection{Theoretical expressions for $\kappa(\theta)$}

As it has been stated on the previous section, the convergence profile is one of the physical observables for both magnification and gg-lensing. Thus, in order to connect the measurements with a cosmological model, its dependence on the 
cosmological parameters must be provided. Depending on the nature of the lenses considered -that is, whether the lenses are galaxies, voids, clusters or troughs-, the approach may differ.

On this section, the solutions for galaxies and voids is calculated. A simple solution for troughs can be found on Gruen et al. [96].

The simplest solution for the convergence profile is for a sample of galaxies. Taking into account the definition of $\kappa$

$$
\kappa(\theta)=\left\langle\delta_{g}\left(\hat{n}, z_{L}\right) \kappa\left(\hat{n}^{\prime}, z_{S}\right)\right\rangle_{\theta}
$$

if a linear, constant and redshift-independent galaxy-bias $\left(b_{L}\right)$ is considered, a relation between the galaxy density contrast $\delta_{g}$ and matter density contrast $\delta_{M}$ can be established:

$$
\delta_{g}\left(\hat{n}, z_{L}\right)=b_{L} \delta_{M}\left(\hat{n}, z_{L}\right) .
$$

Thus, the convergence profile is given by

$$
\kappa(\theta)=b_{L}\left\langle\delta_{M}\left(\hat{n}, z_{L}\right) \kappa\left(\hat{n}^{\prime}, z_{S}\right)\right\rangle_{\theta} .
$$

If it is considered that the convergence field $\kappa(\hat{n})$ can be expressed also as a function of the matter density contrast by Equation 2.24 and Equation 2.22:

$$
\kappa(\theta)=b_{L}\left\langle\delta_{M}\left(\hat{n}, z_{L}\right)\left[\int_{0}^{z} d z^{\prime} \frac{r\left(z^{\prime}\right)\left[r(z)-r\left(z^{\prime}\right)\right]}{r(z)} 4 \pi G a^{2} \bar{\rho} \delta_{M}(\hat{n}, z)\right]\right\rangle .
$$

This leads finally to [97]:

$$
\begin{aligned}
\kappa(\theta)=\frac{3 H_{0}^{2} \Omega_{M}^{0}}{2 c^{2}} & \int_{0}^{\infty} d z_{L}^{\prime} \frac{\phi_{L}\left(z_{L}^{\prime}\right)}{1+z_{L}^{\prime}} \int_{z_{L}^{\prime}}^{\infty} d z_{S}^{\prime} \phi_{S}\left(z_{S}^{\prime}\right) \frac{r\left(z_{L}^{\prime}\right)\left[r\left(z_{S}^{\prime}\right)-r\left(z_{L}^{\prime}\right)\right]}{r\left(z_{S}^{\prime}\right)} \times \\
& \int_{0}^{\infty} \frac{d k k}{2 \pi} P_{M}\left(k, z_{L}^{\prime}\right) J_{0}\left[k \theta r\left(z_{i}^{\prime}\right)\right],
\end{aligned}
$$

where $\phi_{L}, \phi_{S}$ are the redshift distributions of the lens and source sample respectively, $P_{M}$ the matter power spectrum of the lens sample and $J_{0}$ is the zero-th order Bessel function.

The calculation of the convergence profile for a void needs to assume a void profile. A solution on General Relativity for voids can be found at [98-100], based on the LTB metric,

$$
\delta\left(r_{v}\right)=\delta_{0} g(a)\left(1-\frac{2}{3} \frac{r_{v}^{2}}{r_{0}^{2}}\right) \exp \left(-\frac{r_{v}^{2}}{r_{0}^{2}}\right)
$$

where $r_{v}$ is the radial comoving distance on the system of coordinates centered at the void, $r_{0}$ the radial size of the void, $\delta_{0}$ the central underdensity and $g(a)$ the growth factor not normalized at present. On the system of coordinates 
centered on the observer and considering non-spherical voids, this leads to the Kovacs \& Garcia-Bellido void-profile (KGB):

$$
\delta_{K G B}(\theta, z)=\delta_{0} g(a)\left[1-\frac{2}{1+2 q^{2}}\left(\frac{r_{\|}^{2}}{r_{0}^{2}}+\frac{q^{2} r_{\perp}^{2}}{r_{0}^{2}}\right)\right] \exp \left[-\left(\frac{r_{\|}^{2}}{q^{2} r_{0}^{2}}+\frac{r_{\perp}^{2}}{r_{0}^{2}}\right)\right]
$$

where $q^{2}=1-e^{2}$ with $e$ the ellipticity of the void and

$$
\begin{gathered}
r_{\|}=r(z) \cos \theta-r\left(z_{v}\right) \\
r_{\perp}=r(z) \sin \theta
\end{gathered}
$$

Here $r(z)$ is the radial comoving distance at redshift $z$, whereas $z_{v}$ is the redshit of the center of the void and $\theta$ the angular separation form the center of the void. From Equation 2.69 and Equation 2.8, the convergence profile around a single void can be obtained:

$$
\kappa(\theta)=\frac{1}{2} \frac{\Sigma_{K G B}(\theta)}{\Sigma_{c}}
$$

where the surface density is given by

$$
\Sigma_{K G B}(\theta)=\int_{0}^{\infty} d z^{\prime} \bar{\rho}_{M}\left(z^{\prime}\right)\left[\delta_{K G B}\left(\theta, z^{\prime}\right)+1\right] .
$$

Here the $\bar{\rho}_{M}\left(z^{\prime}\right)$ is the matter average density of the Universe at redshift $z^{\prime}$, given by

$$
\bar{\rho}\left(z^{\prime}\right)=\frac{\Omega_{M}^{0} \rho_{c}}{\left(1+z^{\prime}\right)^{3}}
$$

with $\rho_{c}$ the critical energy density of the Universe. 


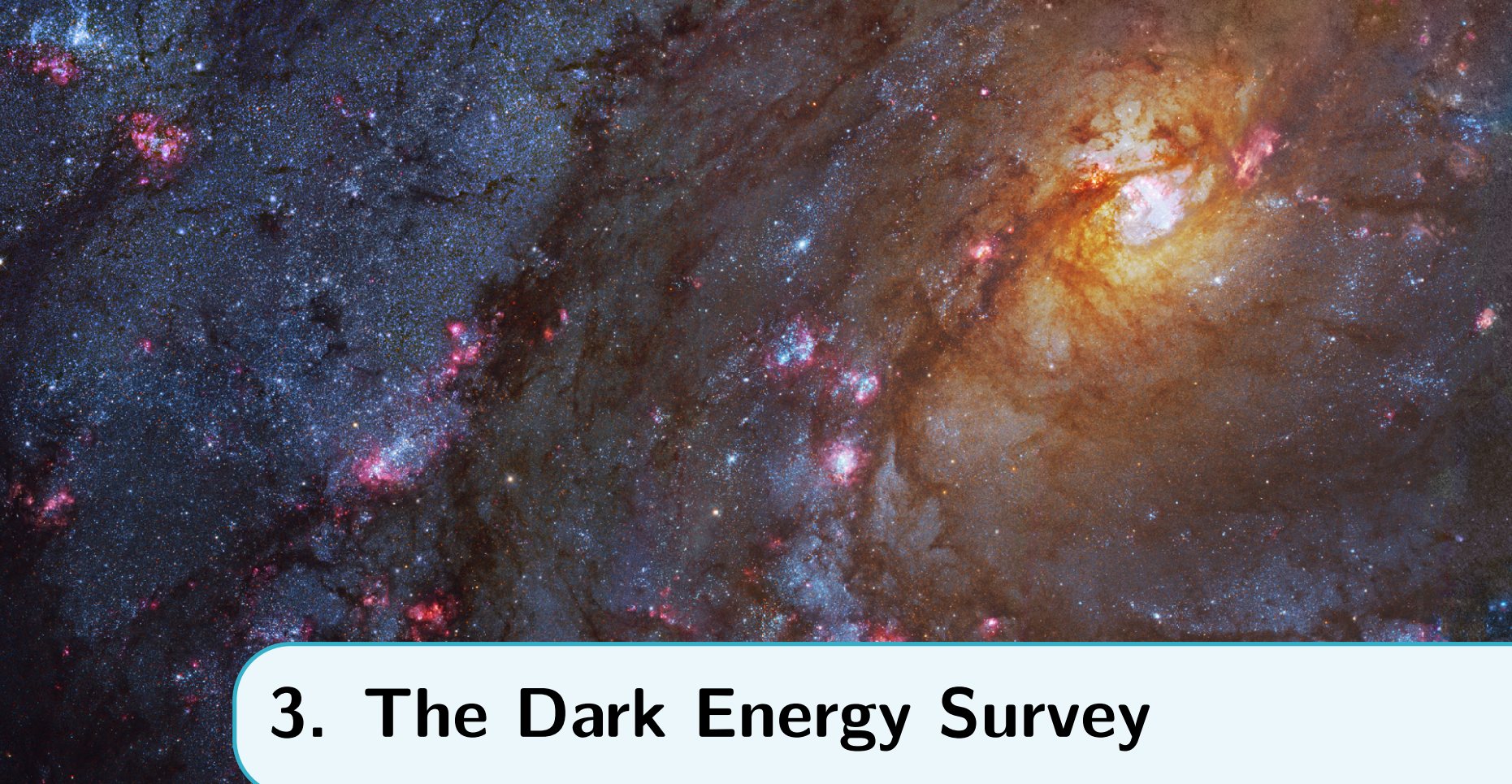

4.8:

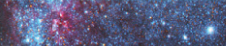

The Dark Energy Survey (DES) [101] is a grizY photometric galaxy survey that has as main scientific goal to shed light on the nature of the Dark Energy. DES uses four probes to unravel the nature of Dark Energy: the number of clusters as a function of redshift, the measurement of the scale of the baryon acoustic oscillations (BAO) peak, the weak gravitational lensing of galaxies and the measurement of the Hubble diagram with type Ia Supernovae (SNIa). By the end of five years of observations, DES will cover $5000 \mathrm{deg}^{2}$ of the Southern Hemisphere up to magnitude $i<24.0$ at the $10 \sigma$ detection level. Taking this into account, this survey is expected to measure 10000 clusters up to redshift 1.0, 200 million galaxy-shapes for weak-lensing (with $z<1)$, 300 million galaxies for $\mathrm{BAO}(z<1.4)$ and 3000 SNIa up to redshift 1.0. The power of DES resides on the combination of all the probes breaking degeneracies on the cosmological parameter phase-space leading to a precision better than the $5 \%$ on the parameters $w_{0}$ and $\Delta w_{a}<0.2$ on the equation of state of the Dark Energy.

DES is an international collaboration formed by about 500 scientists from more than 20 institutions from: USA, Spain, UK, Brazil, Germany, Australia, Chile and Switzerland. The Collaboration has built a very sensitive camera, DECam (see Figure 3.1 and Figure 3.2) [102], that has been mounted at the 4-m Victor M. Blanco Telescope ${ }^{1}$ at the Cerro Tololo Inter-American Observatory (CTIO), located near La Serena (Chile).

\subsection{The DECam}

The DECam (Dark Energy Camera), is the main instrument of the experiment. It is composed mainly by:

- The 570 megapixel focal plane, formed by 70 CCDs.

\footnotetext{
${ }^{1}$ This is the same telescope where Schmidt and Perlmutter performed some of the observations leading to the Nobel Prize in 2010 for the discovery of Dark Energy.
} 
- Low-noise readout electronics.

- Wide-field optical corrector, producing $3 \mathrm{deg}^{2}$ field of view.

- Filter and shutter system.

- Hexapod for stability.

Since DES is going to observe very highly redshifted galaxies, the camera's CCDs have been specifically designed at Lawrence Berkeley National Laboratory to detect red light. In order to do so, the silicon layer is ten times thicker $-250 \mu \mathrm{m}$ - than conventional ones ${ }^{2}$. This results in a quantum efficiency $>80 \%$ on the $600-950 \mathrm{~nm}$ range, $>60 \%$ on the $400-600 \mathrm{~nm}$ and $>50 \%$ on the $900-1000 \mathrm{~nm}$. The DECam focal plane consist of the following types of CCDs:

- Science array: formed by 62 CCDs with $2048 \times 4096$ pixels. Each pixel is $15 \mu \mathrm{m}$ of side that, at the prime focus fot the Blanco Telescope, results on a pixel scale of 0.27 arc-seconds on the sky.

- Four $2048 \times 2048$ guider CCDs.

- Eight $2048 \times 2048$ focus and alignment CCDs.

To minimize the noise and dark currents due to the electronics system, DECam operates on an environment cooled by liquid nitrogen at $180 \mathrm{~K}$ and a vacuum of $\sim 10^{-9} \mathrm{~atm}$. The whole readout process takes 17 seconds (about the same as the slewing-time of the telescope). Readout and clocking electronic boards were produced and designed in Spain at CIEMAT and IFAE.

\subsection{Survey strategy}

The total amount of time awarded to DES at CTIO for observing the total area to the nominal depth on the five photometric bands is of 525 nights over a 5 -year period. The rest of the nights, DECam is available to the scientific community. The tank-shaped footprint, that can be seen at Figure 3.3 is not casual but is optimized for the several probes.

- The cannon located at the equator, is known as stripe-82 and overlaps with several spectroscopic surveys such as SDSS, to calibrate the photometric redshifts (photo-z hereafter).

- The rounded shape - the body- is intended to have the largest possible scales for BAO measurement.

- The lower part - the wheels - is designed to overlap with the South Pole Telescope (SPT) to measure the Sunyaev-Zel'dovich effect correlations with CMB.

The DES observations can be split in two: the transient survey and the wide-field survey.

\section{The transient survey}

The transient survey is designed to measure SNIa. Selected small portions of the sky -known as the supernovae fields- are surveyed periodically to time to look for supernovae explosions and measure its luminosity curve as a function of time. Although it is designed to SNIa astronomy, some ancillary Solar-System astronomical results have been reported, such as Jupiter-trojans and trans-Neptunian

\footnotetext{
${ }^{2}$ Sensitivity to long wavelengths is increased when passing trough more silicon.
} 


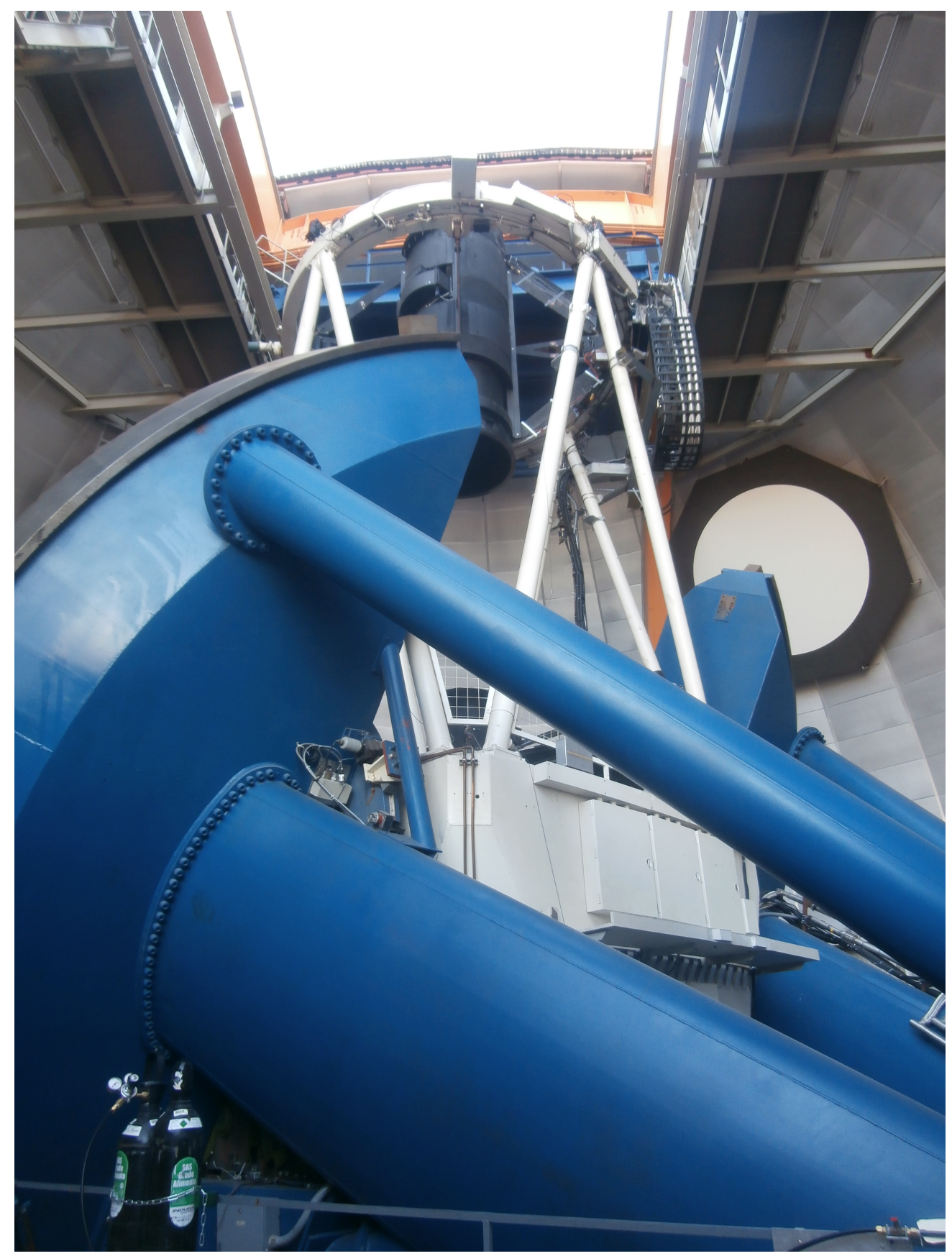

Figure 3.1: DECam mounted at the focus of the Victor Blanco Telescope. Image credit: M. Garcia-Fernandez 


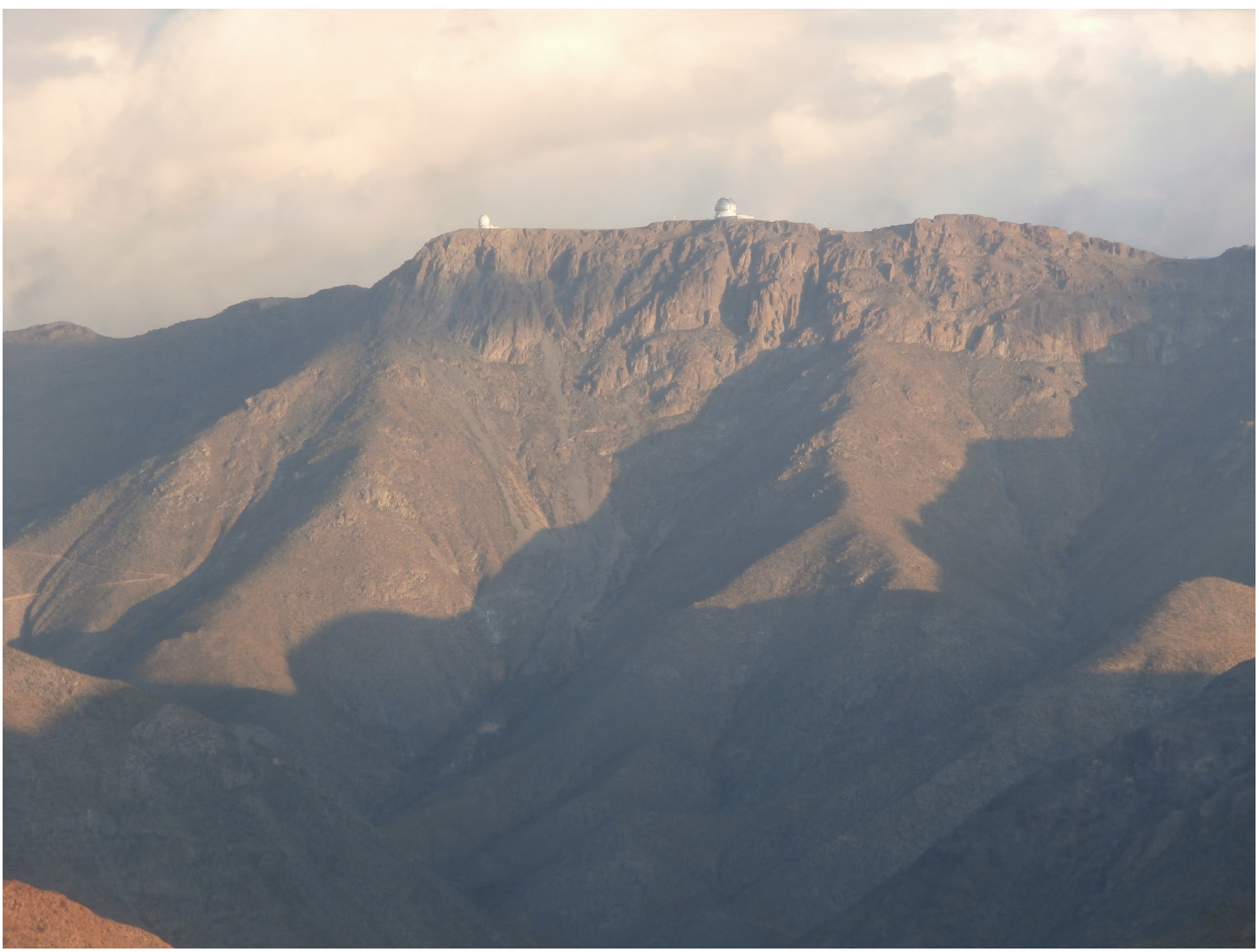

Figure 3.2: Location of the 4-m Victor Blanco Telescope at Cerro Tololo. Chilean Andes. Image credit: M. Garcia-Fernandez

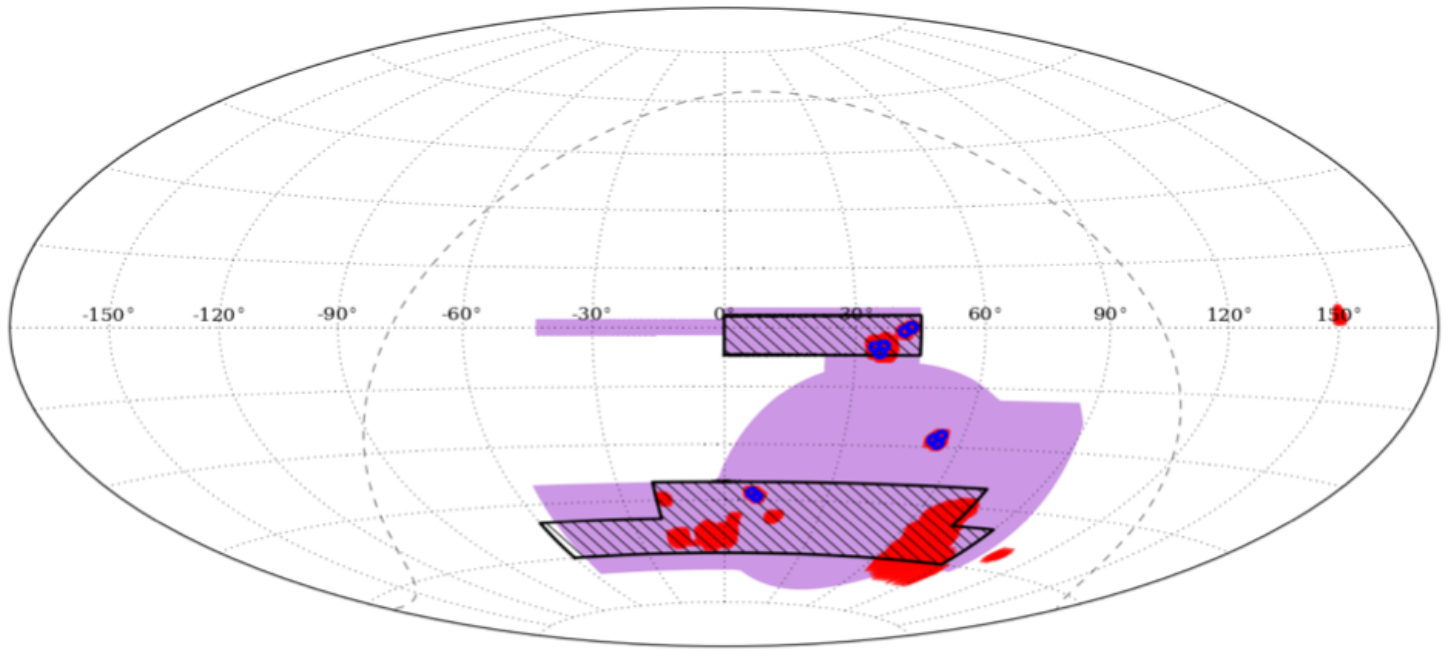

Figure 3.3: DES footprint on equatorial coordinates. Purple area is the total area that DES will cover at the end of the five years (Y5). Red areas -that overlap with the purple- are the Science Verification observations. Shaded areas are the first year campaign of observations (Y1). Dark blue regions are the SNIa fields. Dotted line represent the galactic plane. Image credit: The DES Collaboration. 
detection and searches for the recently proposed planet-9 [103]. These ancillary physics also use the wide-field to increase the area.

\section{The wide-field survey}

The wide field survey is intended for the rest of the dark energy probes. The observed area is visited 10 times on each band along the 5-year-period to reach the full depth and the maximum level of homogeneity.

\section{Nightly operations at CTIO}

A typical night of observations, if the sky is not overcast and no earthquake threatens the life of the observers, starts in the afternoon taking calibration data on CCDs. Then, after the evening twilight, three standard stars are imaged to calibrate the photometry. These, are well known stars with very well defined and measured photometric properties. After that, the wide-field survey starts. When the supernovae fields are visible -and time requirements are fulfilled-, they are surveyed, returning to the wide-field survey when they are done. Some time before the morning twilight, another three standard stars are imaged, finishing the night. All nightly operations follows the same pattern except if some transient alarm us received. In this case, DES points to the place where the transient has been produced to look for an optical counterpart.

\subsection{The data reduction pipeline}

The data reduction that goes from images to science-ready catalogs of galaxies is carried out at the $\mathrm{NCSA}^{3}$. The first step is to calibrate the data. Then, the different exposures of the same region of the sky for a given band -single-epoch imagesare combined into a single image on a procedure called co-addition -multi-epoch image-. This procedure allows the increase of the observed depth respect to each individual single-epoch image (Figure 3.4). Nevertheless, to reach the DES nominal depth, images are detected on the $r+i+z$ multi-epoch images. This multi-epoch images will constitute the measurement images for each band. Co-addition of the objects is made with the software SwARP [104] and the detection and photometric measurements is made with SExTRACTOR [105] in dual mode. IM3SHAPE [106] and NGMIX [107] packages are used for specific needs like shapes for shear and precise photometry.

\subsection{Current status and latest results}

The Dark Energy Survey began its journey in 2005 with the construction of DECam, starting the data acquisition on 2012 with the Science Verification period. By the end of February 2017, the Year 4 observation campaign has ended (Figure 3.5). The Year 3 reduction pipeline from images to galaxy-catalogs has just finished and is still under inspection, so the most recent data-set that is being used for Cosmology analysis, is the Year 1 release (Figure 3.6 and Figure 3.7).

\footnotetext{
${ }^{3}$ National Center for Supercomputing Applications. Illinois (USA).
} 


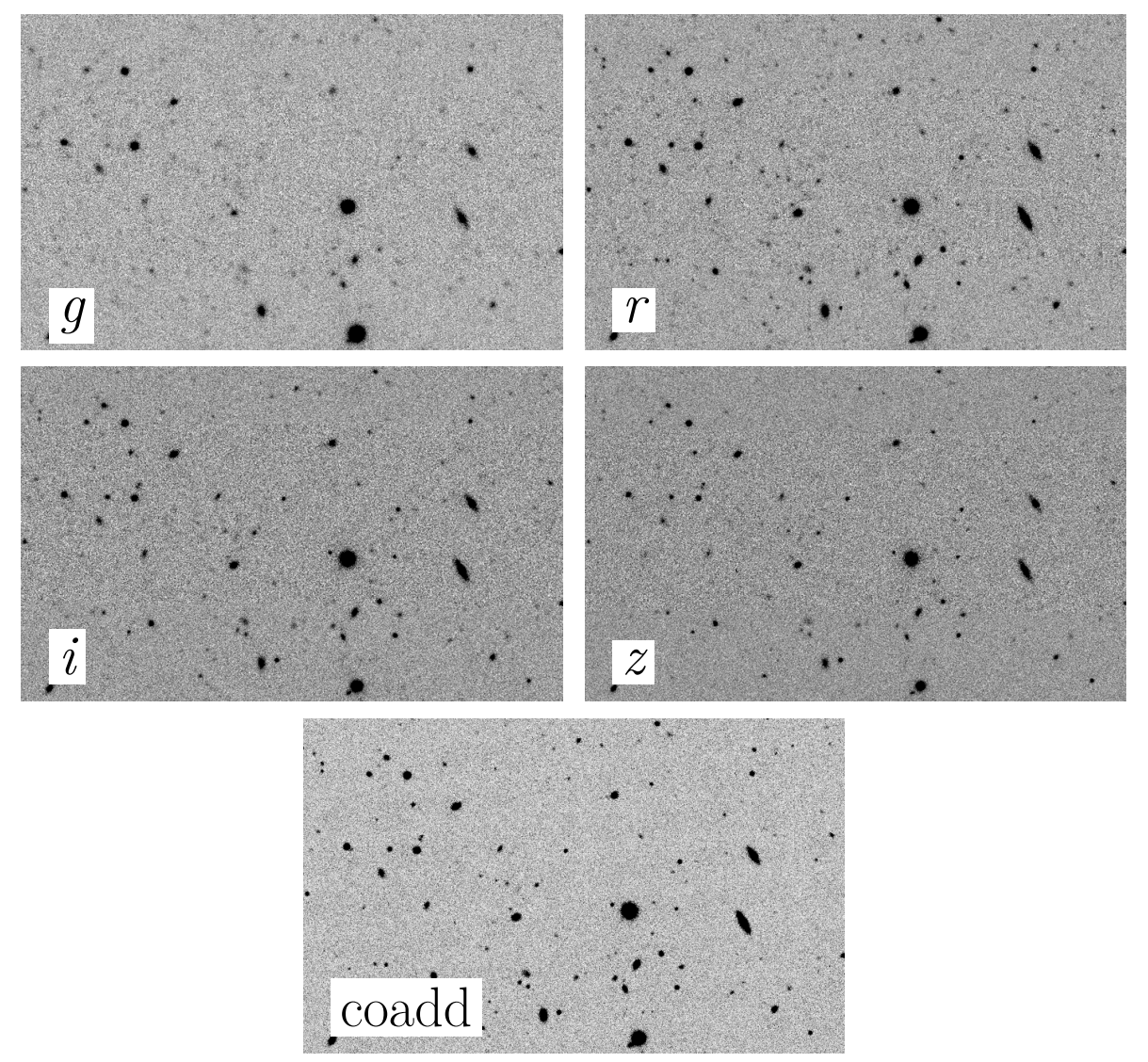

Figure 3.4: Comparison of the multi-epoch image for the griz bands with the detection coadd. Images are taken from DES-database for a region of the tile DES0419-4914 after the Y1 epoch. Image credit: M. Garcia-Fernandez \& The DES Collaboration. 


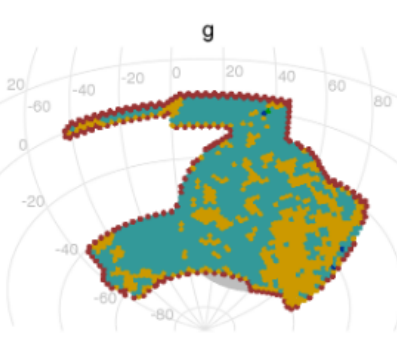

z

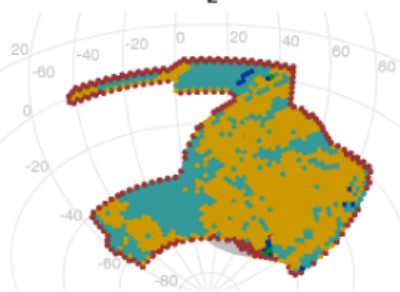

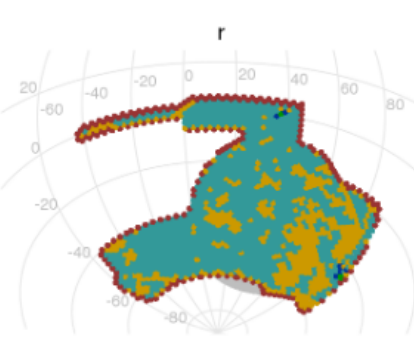

Y

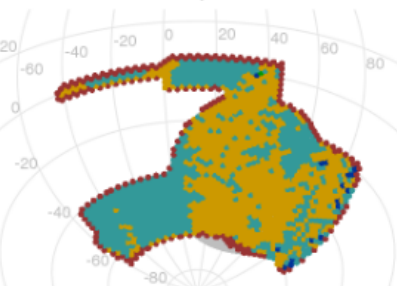

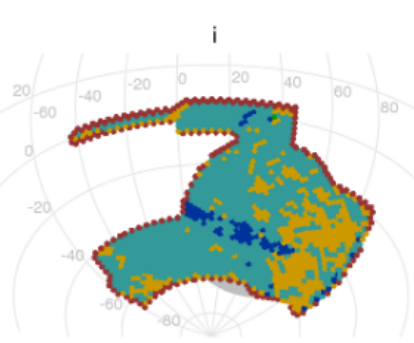

tilings

$-5$

6

- 7

- 8

- $9+$

Figure 3.5: DES coverage at the end of Year 4 observations campaign for the grizY photometric bands. All the area has at least 6 tiles out of 10. It can be also seen that there is plenty of area with 7 tiles. Image credit: The DES Collaboration.

Currently, no precise constrains on dark energy have been made yet, since they require an extensive and demanding control of systematic errors that is still ongoing. Nevertheless several other works on Cosmology have been provided, such as strong-lensing [74, 108, 109], Sunyaev-Zel'dovich [110], voids and troughs $[96,111]$, tests of log-normality [112], clusters [113], weak-lensing [114-120] and large-scale-structure correlations with CMB [116, 121, 122].

Constrains on the cosmological parameter space provided by DES are based on the Science Verification shear analysis [123, 124]. Nevertheless, the most powerful measurement is produced by the combination of clustering with gg-lensing [125] on the $\sigma_{8}-\Omega_{M}$ plane. Although results provided are not yet competitive, it is a remarkable milestone for DES to provide such results with just the $3 \%$ of the planed total area (Figure 3.8).

But not everything is about dark energy at DES. Several other results has been provided [126]: discovery of several trans-neptunian objects (TNOs), Jupitertrojans and main belt asteroids, characterization of variable stars, detection and characterization of Milky-Way satellite galaxies -and its use to put constrains on dark matter- and gravitational-wave follow-up. 


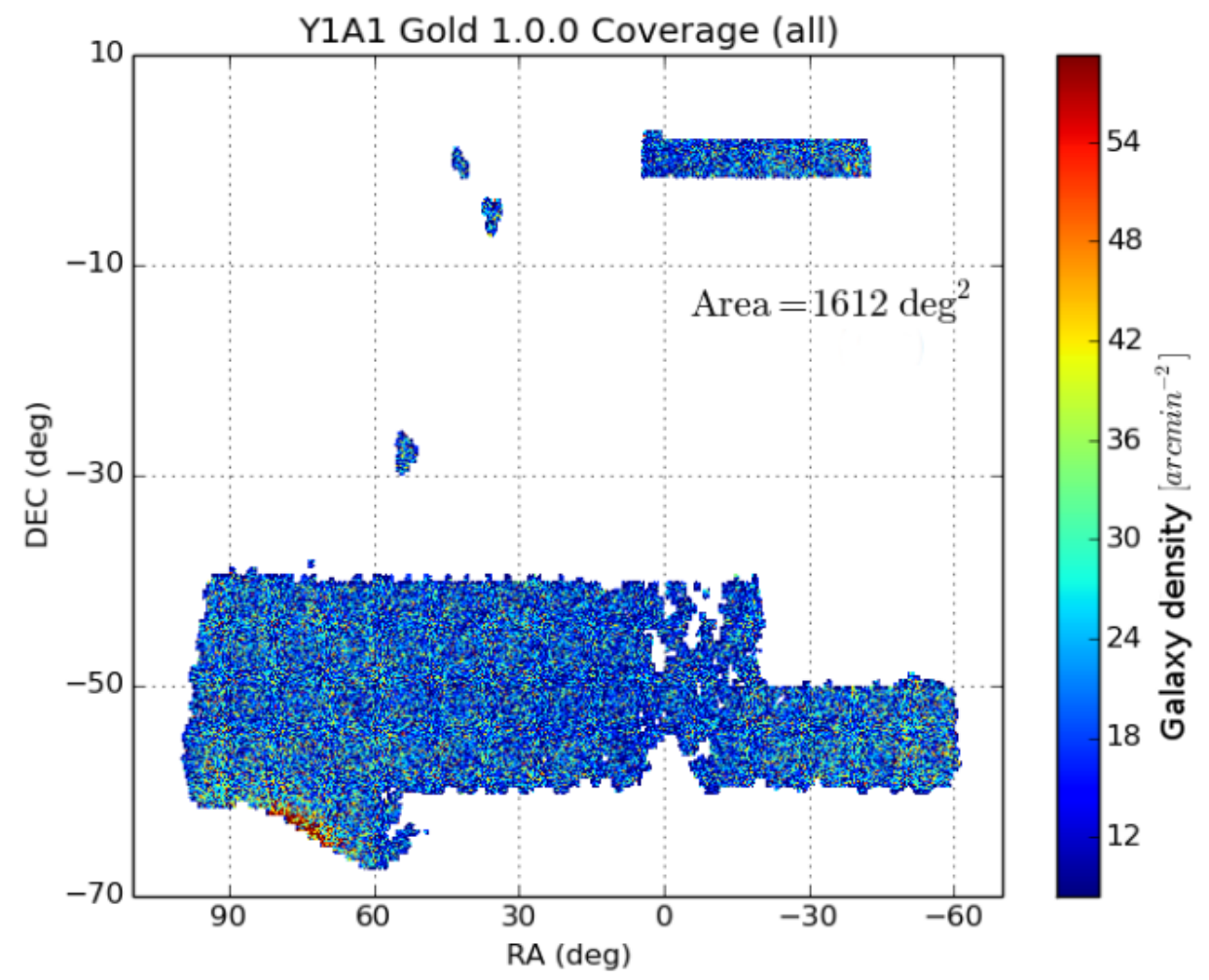

Figure 3.6: DES Year 1 spatial distribution of objects on equatorial coordinates. Image credit: The DES Collaboration.

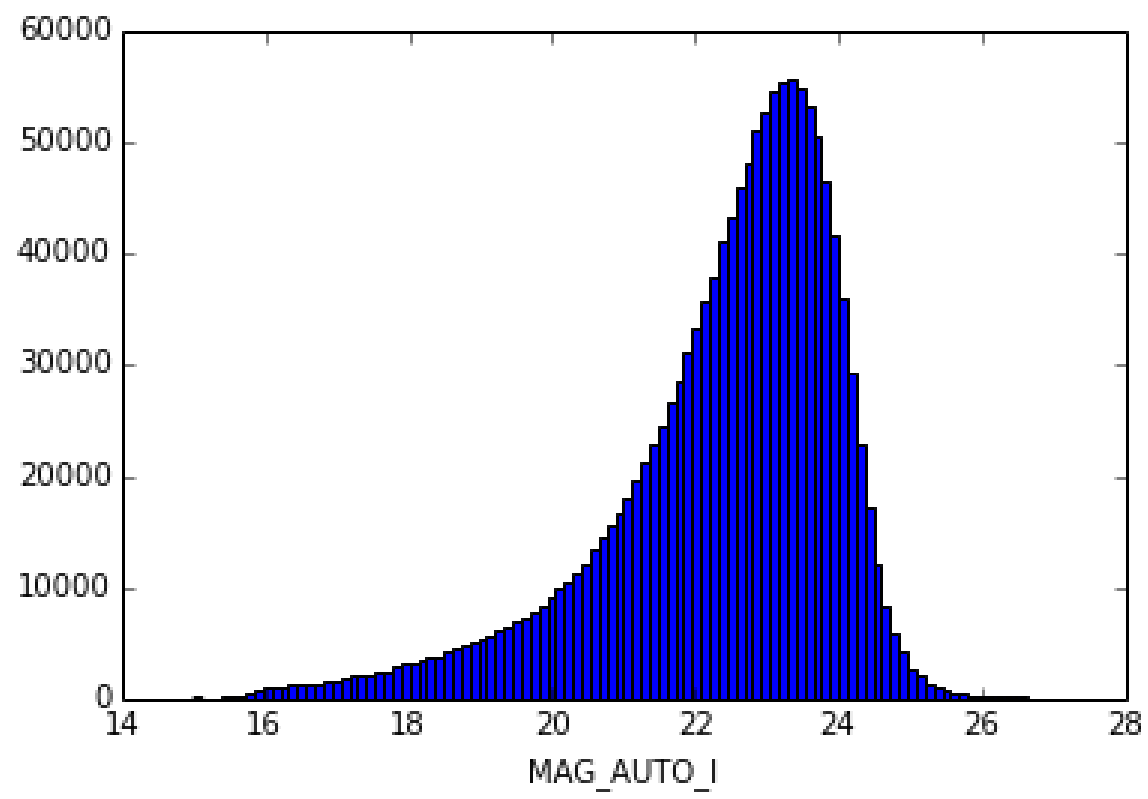

Figure 3.7: DES Year 1 magnitude distribution of objects on the $i$-band (arbitrary normalization). The average depth reached is $i \sim 22.8$. Image credit: The DES Collaboration. 

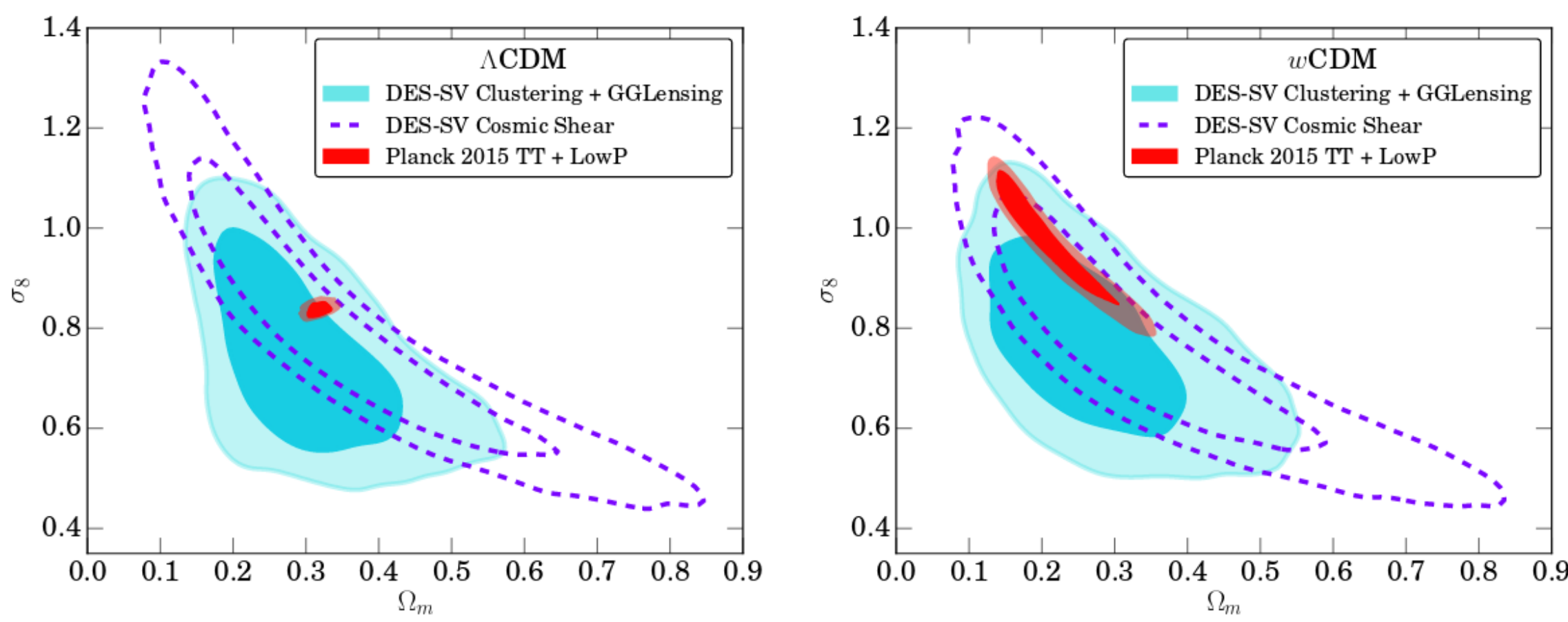

Figure 3.8: DES-SV constrains on the $\Omega_{M}-\sigma_{8}$ plane combining clustering and gg-lensing [125]. Two scenarios are considered: $\Lambda$ CDM and the presence of non-evolving dark energy ( $w \mathrm{CDM})$. 



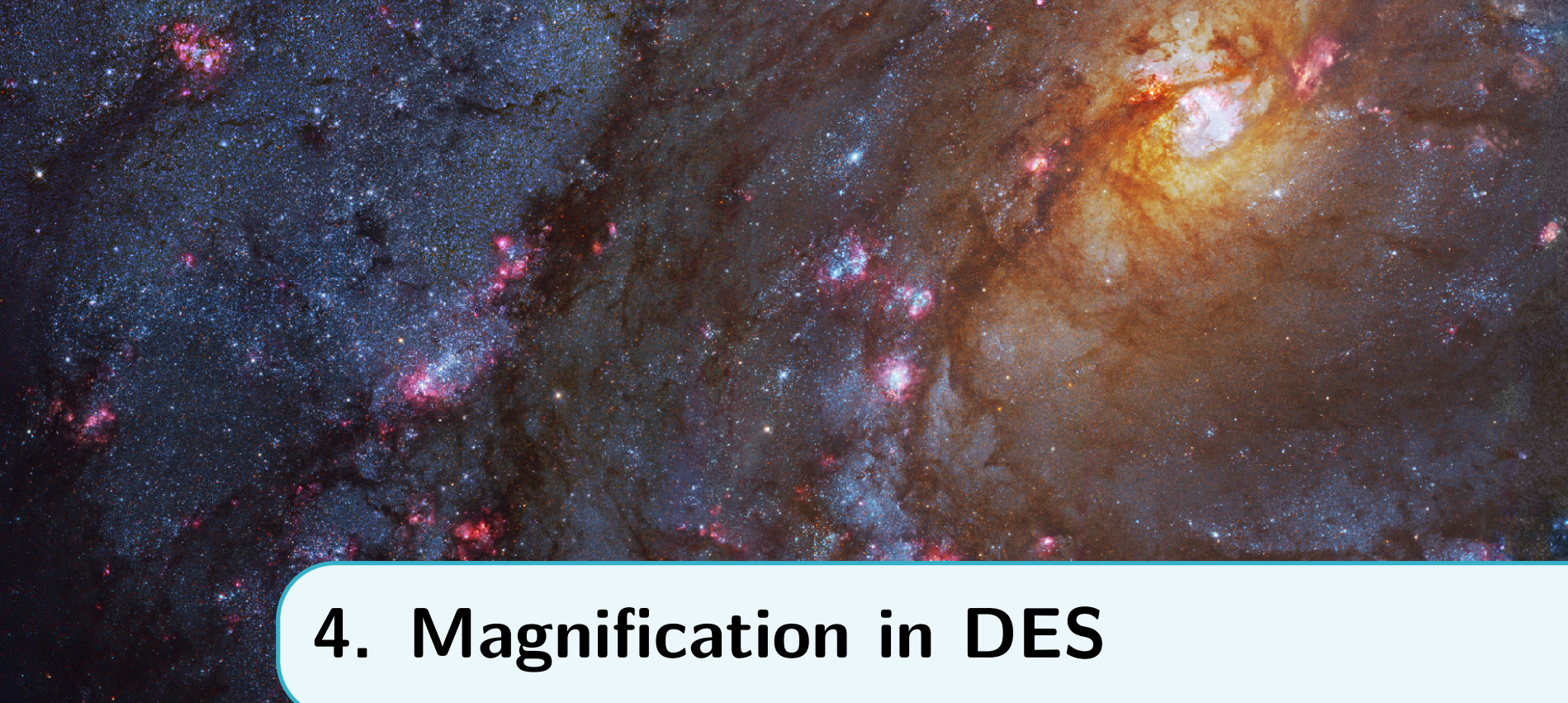

45

Extensive wide-field galaxy surveys have allowed accurate measurements of weaklensing effects. Previous magnification measurements involved the use of very massive objects as lenses, such as luminous red galaxies (LRGs) and clusters [127-130], or high redshift objects as sources, such as Lyman break galaxies (LBGs) [131, 132], quasars (QSOs) [133-138] and sub-mm sources [139] to improve signalto-noise ratio. In addition to the number count technique used on this Thesis, other observational effects produced by magnification have been measured as well: the shift in magnitude [95], flux [140] and size [93].

On this chapter, first, the methodology to measure magnification is described (section 4.1) and validated with simulations (section 4.2). Then, the methodology is used to measure the magnification signal at the Dark Energy Survey Science Verification data (SV; section 4.3). Finally, it is employed to measure the convergence profile of voids and troughs with the Year 1 data (Y1; section 4.4).

It is worth remarking that, as it was defined in chapter 2, given both the redshift of the lenses and the sources, the convergence is a two-dimensional scalar field that is independent of the selected lens or source sample. Nevertheless, by choosing a suitable lens sample, different parts of the log-normal distribution of the convergence field [112] can be probed, leading to a different convergence profile.

\subsection{Measuring Magnification through Number Count}

As it was described in chapter 2, the amplitude of the magnification signal depends on two factors: the magnitude number-count slope parameter $(\alpha-1)$ and the lensing kernel, which for a given lens sample, is a function of the redshift of the source sample. LBGs and QSOs have been traditionally used on magnification studies as sources since they have steep magnitude distribution, leading to a high value of $\alpha-1$. In addition, this population of galaxies is located at very high 
redshift $(2 \lesssim z \lesssim 4)$, leading to a high lensing efficiency and a clean redshift separation between the lens and source samples. Nevertheless, this population of galaxies has very low density, feature that can prevent the measurement of a magnification signal for small-area surveys. In addition, the selection of a population of LBGs involves the method known as dropout technique, that requires the development of a custom data-reduction pipeline just to select this specific population of galaxies. For large-area surveys such as DES and LSST, the amount of computing time to run the data-reduction pipeline makes this approach very inefficient, and alternative methods must be developed.

The caveats related to the use of LBGs and QSOs as sources can be avoided by using the whole measured galaxy population. Then, only redshift cuts are imposed in order to separate the lens and source samples. This approach has the advantage that the density of the source sample is higher, reducing significantly the shot-noise. Nevertheless, the accuracy on the determination of the redshift on broad-band galaxy-surveys -such as DES- is limited, introducing an important source of systematic errors that must be avoided and carefully taken into account.

In addition to the lens-source redshift overlap, magnification suffers from several systematic errors based on the photometry [141, 142], such as: depthinhomogeneities, the zero-point correction and calibration, or possible shifts on the magnitude determination due to inaccurate sky-background subtraction. The important reduction of shot-noise on wide-field surveys requires the development of new techniques to estimate and control these sources of systematic errors.

The use of the general population of galaxies on small-area surveys is mandatory to reach a significance that allows the detection of the magnification signal. On wide-area surveys the use of this population is not necessary although new studies can be made if it is used. Having a very dense population of galaxies as sources allows to use a set of lenses that are less numerous: voids and troughs. This allows to produce new physics analysis.

The usual approach that can be found on the literature to measure magnification is the optimal weighting technique [97]. This methodology can be summarized as follows:

1. Split data sample into two well-separated photo-z bins, termed lens and source. Splitting must be done minimizing the overlap between the true redshift distributions of the samples. Otherwise, by Equation 2.28, an additive signal is introduced.

2. Weight each source galaxy by its optimal weight.

3. Compute the two-point angular cross-correlation between the lens and the unique source sample.

The weight of the $i$-th galaxy $\left(w_{i}\right)$ is given by:

$$
w_{i}=\alpha_{S}\left(m_{i}\right)-1
$$

where $\alpha_{S}\left(m_{i}\right)$ is the number-count slope given by Equation 2.35 and $m_{i}$ is the 
magnitude of the $i$-th galaxy. This procedure allows to obtain the maximum signal-to-noise. Nevertheless, this weighting makes it hard to disentangle the impact of the different systematic effects on the measured signal.

As an alternative approach the following procedure is proposed and implemented:

1. Split the data sample into two well-separated photo-z bins, termed lens and source. Splitting must be done minimizing the overlap between the true redshift distributions of the samples. Otherwise, by Equation 2.28, an additive signal is introduced.

2. For each photometric band, define several subsamples from the source sample using different values for the maximum (threshold) magnitude. This is made in order to trace the evolution of the amplitude of the magnification signal with the number count slope (see Equation 2.35).

3. Compute the two-point angular cross-correlation function between the unique common lens sample and each source subsample for each band.

Once the two-point angular correlation function has been measured, it can be compared with theoretical predictions as described in chapter 2 allowing the desired parameter constraints.

As it has been stated previously, the amplitude of the measured cross-correlation function depends on the shape of the galaxy number count distribution. Nevertheless, due to this shape -for a fixed footprint, population of galaxies and redshift distribution-, the brighter is the magnitude limit of the sample, the bigger is the amplitude of the two point angular cross correlation function. However, the number of bright galaxies is lower than the number of faint galaxies [143], so shot noise becomes more important increasing their measurement uncertainties. For this reason, there exists a magnitude cut that is a trade-off between amplitude and shot noise, maximizing the signal-to-noise ratio. In order to find the optimum magnitude cut for a given sample, define the signal-to-noise ratio for a given angular range and magnitude cut $m^{\prime}<m$ can be defined as [144]:

$$
\frac{S}{N}(m)=\frac{\left\langle\omega_{L S}(\theta ; m)\right\rangle}{\left\langle s\left(\omega_{L S}(\theta ; m)\right)\right\rangle},
$$

where $\left\langle s\left(\omega_{L S}(\theta ; m)\right)\right\rangle$ is the average shot noise of the two point angular cross correlation functions and the averages are extended to the angular range considered in the analysis. The shot noise for a given angular aperture is given by the number of pairs inside each angular bin as

$$
\sigma\left(\omega_{L S}(\theta ; m)\right)=\frac{1}{\sqrt{P_{L S}(\theta ; m)}}
$$

where $P_{L S}(\theta ; m)$ is the number of pairs from the lens-source samples separated by an angular distance $\theta$ for a magnitude cut $m^{\prime}<m$. The number of pairs per angular bin is given by the product of the number of source galaxies that fall inside a given annulus times the number of sources inside that annulus. Considering, as 
a first order approach, that the samples are uniform, the number of lens-source pair-counts of galaxies for a bin centerd at $\theta$ with solid angle $\Delta_{\Omega}$ is given by

$$
P_{L S}(\theta ; m)=\left[\frac{N_{L}}{A} \Delta_{\Omega}(\theta)\right]\left[\frac{N_{S}(m)}{A} \Delta_{\Omega}(\theta)\right] .
$$

Here $A$ is the solid angle subtended by the dataset, $N_{L}$ is the number of objects at the lens sample and $N_{S}(m)$ the number of objects in the source sample with magnitude limit $m$. Combining Equations 2.36, 4.2 and 4.4, results finally in

$$
\frac{S}{N}(m)=\left\langle\omega_{0}\right\rangle[\alpha(m)-1] b_{L} \frac{\Omega}{A} \sqrt{N_{L} N_{S}(m)},
$$

where $\Omega$ is the solid angle subtended by an annulus with edges the maximum and minimum scales considered. Thus, for a sample, given size, magnitude and redshift distributions -assuming a cosmology- the signal-to-noise ratio can be estimated. Nevertheless, Equation 4.5 assumes that the angular bins are uncorrelated and should be taken as an upper bound to the signal-to-noise. Although this expression does not take into account the full covariance, the behavior

$$
\frac{S}{N} \sim[\alpha(m)-1] \sqrt{N_{S}(m)}
$$

is independent of cosmological and covariance assumptions up to a constant factor, allowing us to use this expression for finding the optimal cut that maximizes the signal-to-noise ratio. Equation 4.6 finds the magnitude cut with the maximum signal-to-noise for a given source sample.

\subsection{Magnification in the MICE-GC simulation}

In order to test the methodology described above in a controlled environment, isolated from any source of systematic error, it is applied to a simulated galaxy sample, in particular MICECAT v1.0. This mock is the first catalog release of the N-body simulation MICE-GC ${ }^{1}[145-147]$. It assumes a flat $\Lambda$ CDM Universe with cosmological parameters $\Omega_{M}=0.25, \Omega_{b}=0.044, h=0.7$ and $\sigma_{8}=0.8$, using a light-cone that spans one eighth of the celestial sphere. Another advantage of using these simulations is the possibility of studying specific systematic effects, as described in subsection 4.3.3.

Among other properties, MICE-GC provides lensed and unlensed coordinates, true redshift (including redshift space distortions) and DES-griz unlensed magnitudes for the simulated galaxies, along with convergence and shear. Conversion from unlensed magnitudes to lensed magnitudes can be done by applying $m_{\mu}=m_{0}-2.5 \log _{10}(1+2 \kappa)$.

Having two sets of coordinates and magnitudes, one in a 'universe' with magnification and another without magnification, allows us to follow the methodology described in section 4.1 for both cases, serving as a test-bench to measure the sensitivity of the method to the magnification effect. The full $5000 \mathrm{deg}^{2}$ of the

\footnotetext{
${ }^{1}$ www.ice.cat/mice
} 


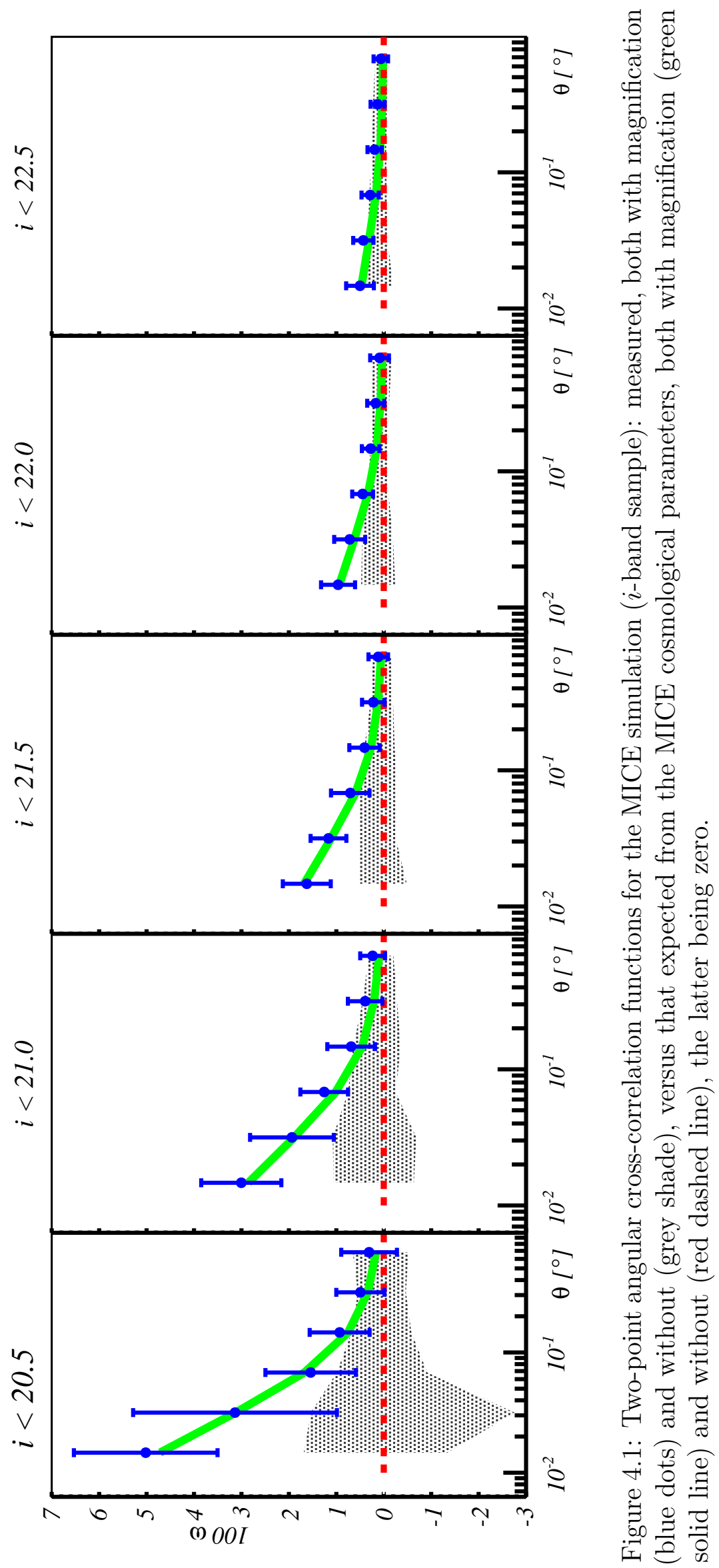


MICE simulation are used, but to match as much as possible the conditions of the DES-SV data, the magnitude cuts described in subsection 4.3 .1 are applied to the lens and source samples. The covariance matrices of data (see section 4.3) are used, in order to match the errors in the DES-SV sample.

In Figure 4.1, the results of the magnification analysis in the MICE simulation for the cases with and without magnification can be seen compared with the theoretical expectations. The methodology used in this work clearly allows us to distinguish both cases for a data-set similar to that of the DES-SV data. Nevertheless, results obtained with the MICE simulation can not be directly extrapolated to SV data to estimate the expected significance because the density of galaxies on the simulation is a factor $\sim 3$ smaller than on the SV data. Also, the luminosity function of the simulation is slightly different from the DES data, which has a direct impact on the number count slope and, consequently, on the amplitude of the measured signal.

\subsection{Magnification in DES Science Verification data}

As of January 2014 -when I started my PhD-, the only data available at DES was the Science Verification data. The first data-release from DES. This dataset was taken just for testing purposes and in order to explore the capabilities of the experiment. Thus, although the nominal depth of the survey was reached, only $\sim 150 \mathrm{deg}^{2}$ where taken. Taking this into account, only $10^{4}$ LBGs are expected at the full DES-SV data, preventing the measurement of magnification with the usual approach. Thus, in order to be capable to reach a detection of the magnification signal with the DES-SV data, the general population of galaxies was selected both as lens and source sample.

The goal of this analysis is to detect a weak-lensing magnification signal and develop methodology to mitigate systematic errors. The data sample is described in subsection 4.3.1. Then, the analysis is described and the results discussed in subsection 4.3.2 following the analysis of the possible systematic errors in subsection 4.3.3. Finally a discussion on the analysis can be found in subsection 4.3.4.

\subsubsection{Data sample}

From the DES SVA1-Gold ${ }^{2}$ main galaxy catalog [148], the largest contiguous field is selected, the SPT-E. Regions with declination $<-61^{\circ}$ are removed in order to avoid the Large Magellanic Cloud. MODEST_CLASS is employed as star-galaxy classifier [149].

The following color cuts are made in order to remove outliers in color space:

- $-1<g-r<3$,

- $-1<r-i<2$,

- $-1<i-z<2$;

\footnotetext{
${ }^{2}$ des.ncsa.illinois.edu/releases/SVA1
} 
where $g, r, i, z$ stand for the corresponding MAG_AUTO magnitude measured by SEXTRACTOR [105].

Regions of the sky that are tagged as bad, amounting to four per cent of the total area, are removed. An area of radius 2 arcminutes around each 2MASS star is masked to avoid stellar halos $[138,150]$.

The DES Data Management [151-153] produces a MANGLE ${ }^{3}$ [154] magnitude limit mask that is later translated to a $N_{\text {side }}=4096$ HEALPIX $^{4}$ [155] mask. Since the HEALPIX mask is a division of the celestial sphere with romboid-like shaped pixels of the same area, to avoid boundary effects due to the possible mismatch between the MANGLE and HEALPIX masks, each pixel is required to be totally inside the observed footprint as determined by MANGLE, by demanding

- $r_{\text {fracdet }}=1$,

- $i_{\text {fracdet }}=1$,

- $z_{\text {fracdet }}=1$;

where $r_{\text {fracdet }}, i_{\text {fracdet }}, z_{\text {fracdet }}$ is the fraction of the pixel lying inside the footprint for $r, i, z$ bands respectively.

Depth cuts are also imposed on the riz-bands in order to have uniform depth when combined with the magnitude cuts. These depth cuts are reached by including only the regions that meet the following conditions:

- $r_{\lim }>23.0$,

- $i_{\lim }>22.5$,

- $z_{\lim }>22.0$;

where $r_{\text {lim }}, i_{\text {lim }}, z_{\lim }$ stand for the magnitude limit in the corresponding band, that is, the faintest magnitude at which the flux of a galaxy is detected at $10 \sigma$ significance level. The resulting footprint, as shown in Figure 4.2, after all the masking cuts amounts to $121 \mathrm{deg}^{2}$.

Photometric redshifts (photo-z) have been estimated using different techniques. In particular, the fiducial code used in this work employs a machine-learning algorithm (random forests) as implemented by TPZ [156], which was shown to perform well on SV data [157]. The redshifts of the galaxies are defined according to the mean of the probability density functions given by TPZ $\left(z_{\mathrm{ph}}\right)$. Other methods are also employed to demonstrate that the measured two-point angular cross-correlation are not a feature induced by TPZ.

\section{Lens sample}

A unique lens sample is defined by the additional photo- $z$ and magnitude cuts:

- $0.2<z_{\mathrm{ph}}<0.4$

- $18.0<i<22.5$.

These requirements are imposed in order to be compatible with the first redshift bin of the so called 'benchmark sample' [148]. Note that the MAG_AUTO cut along

\footnotetext{
${ }^{3}$ http://space.mit.edu/ molly/mangle/

${ }^{4}$ healpix.jpl.nasa.gov
} 


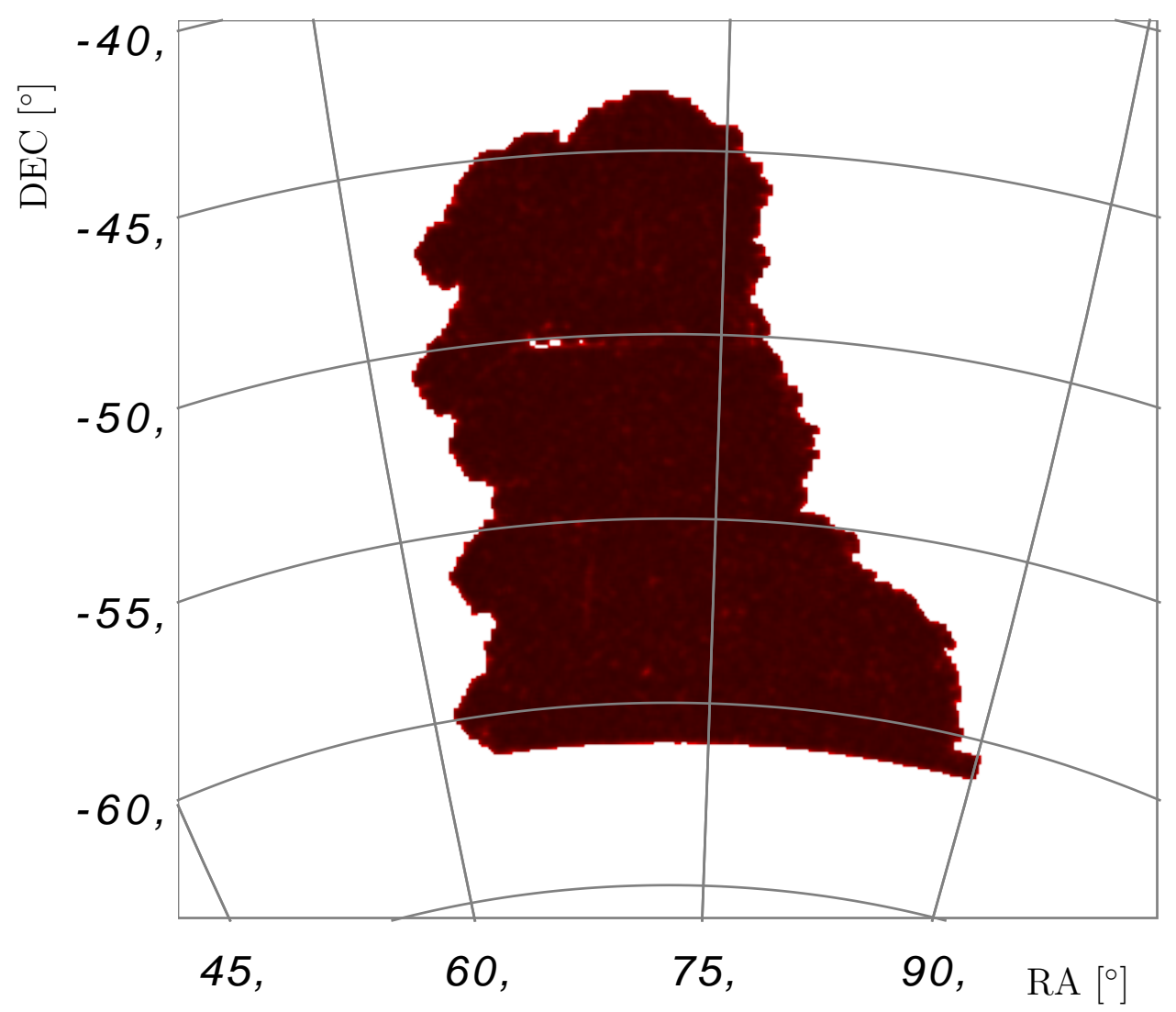

Figure 4.2: Final footprint of the DES SPT-E region after all masking is applied.

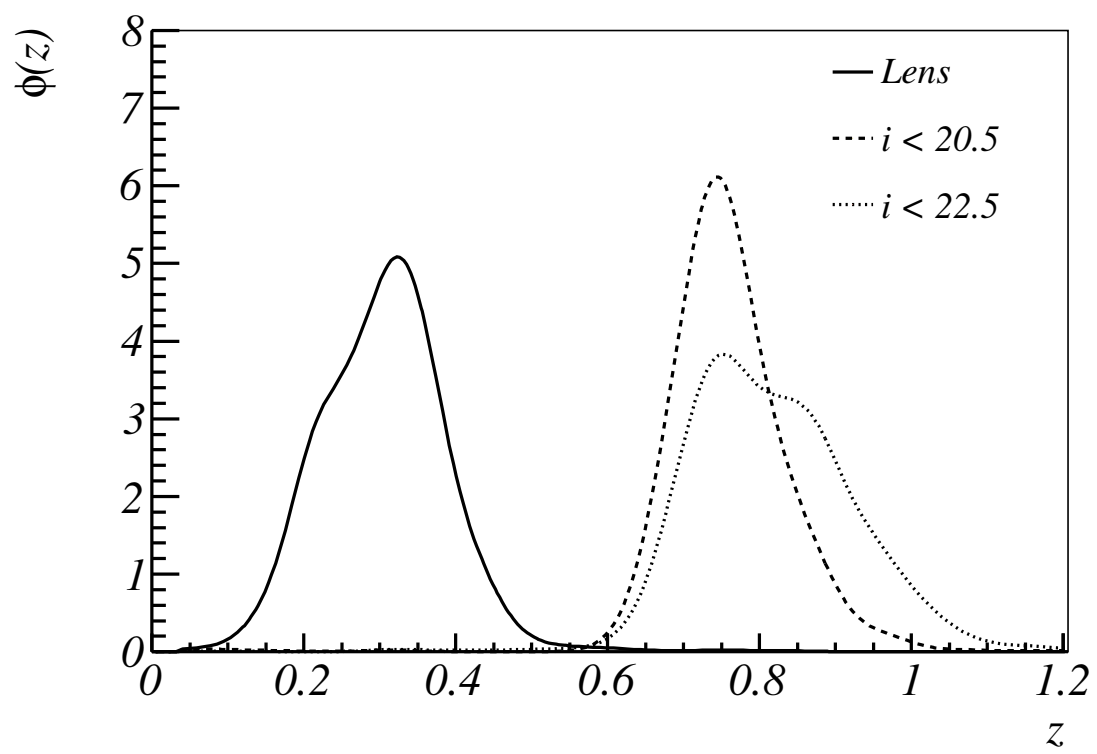

Figure 4.3: Redshift distributions from the stacking of the TPZ probability distribution functions for the lens and two $i$-band sub-samples of the source. 
with the previous $i$-band depth cut guarantees uniformity [148].

\section{Source sample}

Three source samples are defined, one per band:

- R: $0.7<z_{\mathrm{ph}}<1.0$ and $r<23.0$

- I: $0.7<z_{\mathrm{ph}}<1.0$ and $i<22.5$

- Z: $0.7<z_{\mathrm{ph}}<1.0$ and $z<22.0$.

Following the same approach we used on the lens, defined over the 'benchmark' sample, the MAG_AUTO cut along with the previously defined depth cuts also guarantee uniformity on the corresponding band. Within each R, I, Z source sample five sub-samples that map the magnitude evolution are defined,

- $\mathrm{R}_{1}: r<21.0 ; \mathrm{R}_{2}: r<21.5 ; \mathrm{R}_{3}: r<22.0 ; \mathrm{R}_{4}: r<22.5 ; \mathrm{R}_{5}: r<23.0$.

- $\mathrm{I}_{1}: i<20.5 ; \mathrm{I}_{2}: i<21.0 ; \mathrm{I}_{3}: i<21.5 ; \mathrm{I}_{4}: i<22.0 ; \mathrm{I}_{5}: i<22.5$.

- $\mathrm{Z}_{1}: z<20.0 ; \mathrm{Z}_{2}: z<20.5 ; \mathrm{Z}_{3}: z<21.0 ; \mathrm{Z}_{4}: z<21.5 ; \mathrm{Z}_{5}: z<22.0$.

Here $S_{j}$ with $j=1,2,3,4,5$ are the sub-samples of sample $S$ with $S \in\{R, I, Z\}$. In Figure 4.3, the redshift distributions of the lens and source sample are shown. Note that the sub-samples $\mathrm{R}_{5}, \mathrm{I}_{5}, \mathrm{Z}_{5}$ are equal to $\mathrm{R}, \mathrm{I}, \mathrm{Z}$ respectively.

The $g$-band is not used on this analysis because when the same approach is followed and a uniform sample is defined in that band, the number of galaxies of the lens and source samples decrease dramatically. This increases the shot noise preventing the measurement of number count magnification

\subsubsection{Detection of the weak-lensing magnification signal}

To estimate the cross-correlation functions, the tree-code TREECORR ${ }^{5}$ [158] and the Landy-Szalay estimator [159] are used demanding six logarithmic angular bins:

$$
\omega_{\mathrm{LS}_{\mathrm{j}}}(\theta)=\frac{D_{\mathrm{L}} D_{\mathrm{S}_{\mathrm{j}}}(\theta)-D_{\mathrm{L}} R_{\mathrm{S}_{\mathrm{j}}}(\theta)-D_{\mathrm{S}_{\mathrm{j}}} R_{\mathrm{L}}(\theta)}{R_{\mathrm{L}} R_{\mathrm{S}_{\mathrm{j}}}(\theta)}+1,
$$

where $D_{\mathrm{L}} D_{\mathrm{S}_{\mathrm{j}}}(\theta)$ is the number of pairs from the lens data sample L and the source data sub-sample $\mathrm{S}_{\mathrm{j}}$ separated by an angular distance $\theta$ and $D_{\mathrm{L}} R_{\mathrm{S}_{\mathrm{j}}}(\theta), D_{\mathrm{S}_{\mathrm{j}}} R_{\mathrm{L}}(\theta)$, $R_{\mathrm{L}} R_{\mathrm{S}_{\mathrm{j}}}(\theta)$ are the corresponding values for the lens-random, source-random and random-random combinations normalized by the total number of objects on each sample.

Catalogs produced with BALROG ${ }^{6}[160]$ are used as random sample. See section 4.3.3 for a detailed description and discussion on this.

A covariance matrix is computed for each band by jack-knife re-sampling the data taking into account the correlations between the different magnitude cut

\footnotetext{
${ }^{5}$ github.com/rmjarvis/TreeCorr

${ }^{6}$ github.com/emhuff/Balrog
} 
within each band

$$
\begin{array}{r}
C_{\mathrm{S}}\left(\omega_{\mathrm{LS}_{\mathrm{i}}}\left(\theta_{\eta}\right) ; \omega_{\mathrm{LS}_{\mathrm{j}}}\left(\theta_{\nu}\right)\right)=\frac{N_{\mathrm{JK}}}{N_{\mathrm{JK}}-1} \\
\times \sum_{k}^{N_{\mathrm{JK}}}\left[\omega_{\mathrm{LS}_{\mathrm{i}}}^{k}\left(\theta_{\eta}\right)-\omega_{\mathrm{LS}_{\mathrm{i}}}\left(\theta_{\eta}\right)\right]\left[\omega_{\mathrm{LS}_{\mathrm{j}}}^{k}\left(\theta_{\nu}\right)-\omega_{\mathrm{LS}_{\mathrm{j}}}\left(\theta_{\nu}\right)\right],
\end{array}
$$

where $\omega_{\mathrm{LS}_{\mathrm{j}}}^{k}$ stands for the cross-correlation of the $k$-th jack-knife re-sample and $\omega_{\mathrm{LS}_{\mathrm{j}}}$ is the cross-correlation of the full sample. The $N_{\mathrm{JK}}=120$ jack-knife regions are defined by a $k$-means algorithm [161] using Python's machine learning library SCIKIT-LEARN $^{7}$ [162]. In order to get $N_{\text {JK }}$ regions with equal area, the algorithm is trained on a uniform random sample following the footprint of the data demanding $N_{\text {JK }}$ centers. The regions used on the re-sampling are composed by the Voronoi tessellation defined by these centers. These matrices trace the angular covariance as well as the covariances between functions within each band. No covariance between bands is considered, since each band is treated independently on this work. The reduced covariance matrix of the $i$-band is displayed at Figure 4.4. The behaviour is similar for the other bands.

The measured two-point angular cross-correlation functions and the $\Lambda \mathrm{CDM}$ weak lensing theoretical predictions can be found in Figure 4.5, Figure 4.6 and Figure 4.7. The measured correlation functions are found to be non-zero, compatible with $\Lambda \mathrm{CDM}$ and their amplitude evolves with the magnitude cut. The magnitude cuts imposed to guarantee uniform depth make that, for this data, no negative amplitudes are expected.

To compare with theory, Equation 2.36 and Equation 2.67 and have been used assuming Planck 2015 [163] cosmological parameters. The bias of the lens sample has already been measured independently with different techniques: clustering [148], gg-lensing [119], shear [164] and CMB-lensing [121]. From these values the most precise, from [148], is selected $\left(b_{\mathrm{L}}=1.07 \pm 0.08\right)$ and is assumed to be a constant scale-independent parameter. The number count slope parameter $\alpha_{\mathrm{S}}$ is computed by fitting the cumulative number count of the sample $\mathrm{S}$ to a Schechter function [143] on the range of interest

$$
N_{\mu}(m)=A\left[10^{0.4\left(m-m_{*}\right)}\right]^{\beta} \times \exp \left[-10^{0.4(m-m *)}\right],
$$

where $A, m_{*}, \beta$ are the free parameters of the fit. Then $\alpha_{\mathrm{S}}(m)-1$ is computed by applying Equation 2.35, where $m_{\mathrm{j}}$ is the magnitude limit of the $\mathrm{S}_{\mathrm{j}}$ sub-sample on the considered band. In Figure 4.8 the fit and the number count slope parameter for the I sample are shown.

A goodness of fit test of the measured two-point angular cross-correlation function respect to the theoretical predictions for each band is performed:

$$
\begin{gathered}
\chi_{\text {Planck }}^{2}=\sum_{\eta \nu i j}\left[\tilde{\omega}_{\mathrm{LS}_{\mathrm{i}}}\left(\theta_{\eta}\right)-\omega_{\mathrm{LS}_{\mathrm{i}}}\left(\theta_{\eta}\right)\right] \\
C^{-1}\left(\omega_{\mathrm{LS}_{\mathrm{i}}}\left(\theta_{\eta}\right) ; \omega_{\mathrm{LS}_{\mathrm{j}}}\left(\theta_{\nu}\right)\right)\left[\tilde{\omega}_{\mathrm{LS}_{\mathrm{j}}}\left(\theta_{\nu}\right)-\omega_{\mathrm{LS}_{\mathrm{j}}}\left(\theta_{\nu}\right)\right],
\end{gathered}
$$

\footnotetext{
${ }^{7}$ scikit-learn.org
} 


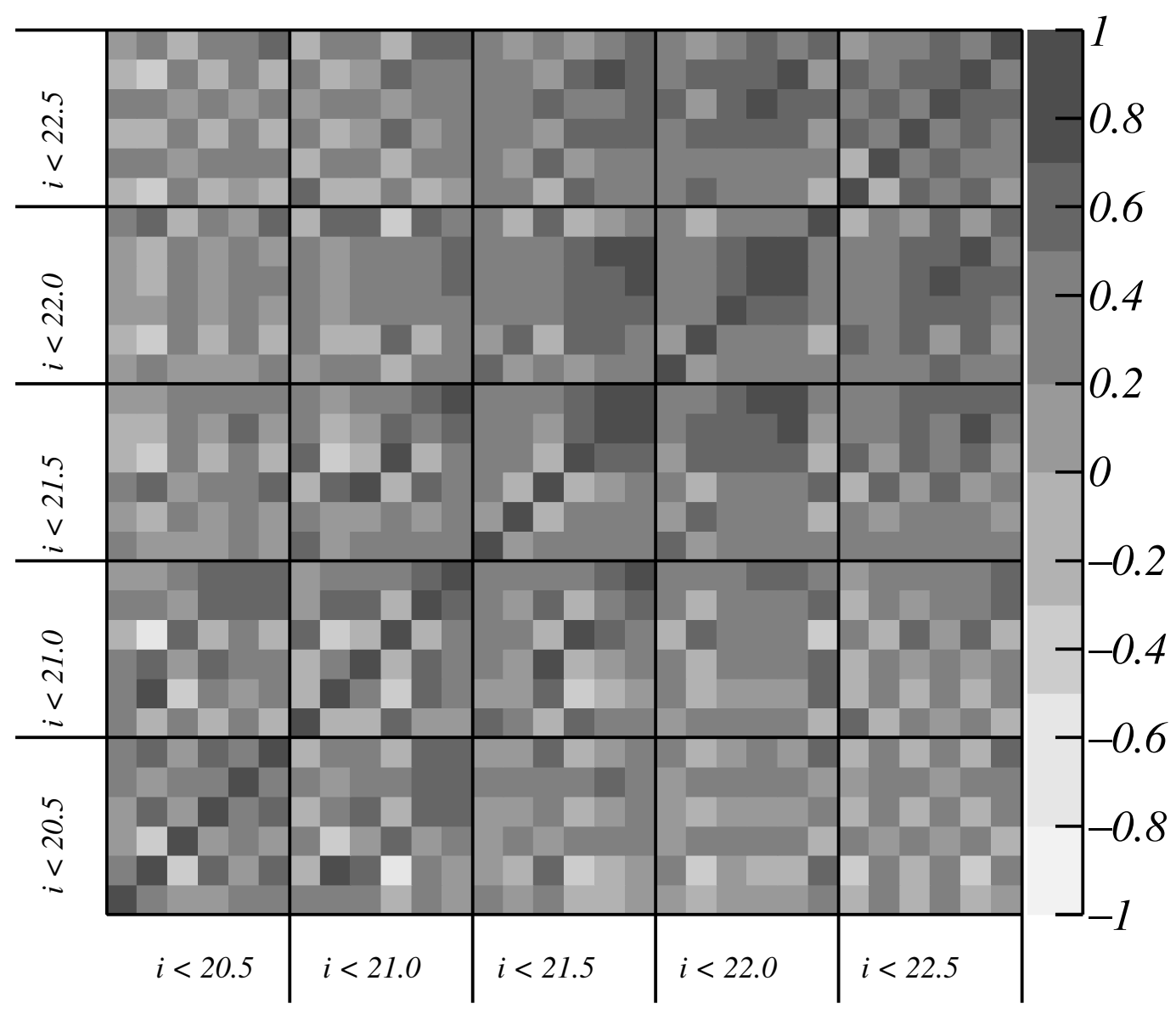

Figure 4.4: Covariance matrix of the $i$-band rescaled by the value of the diagonal $\left(C_{i j} / \sqrt{C_{i i} C_{j j}}\right)$. Each box is the part of the matrix corresponding to the samples labeled at the axis whereas the bins within each box stand for the angular values of the correlation function.

\begin{tabular}{c|c|cc} 
Weight & Sample & $\log _{10} \mathcal{B}$ & $\chi^{2} /$ ndof \\
\hline \multirow{3}{*}{ No } & $\mathrm{R}$ & 3.9 & $21.6 / 30$ \\
& $\mathrm{I}$ & 3.4 & $23.9 / 30$ \\
& $\mathrm{Z}$ & 3.4 & $36.8 / 30$ \\
\hline \multirow{3}{*}{ Yes } & $r<23.0$ & 3.2 & $3.2 / 6$ \\
& $i<22.5$ & 2.1 & $2.1 / 6$ \\
& $z<22.0$ & 2.3 & $2.3 / 6$
\end{tabular}

Table 4.1: Significance of the detection of a magnification signal. Results are shown for the combination of the five subsamples within each band as well as for the faintest sample with weighting. 


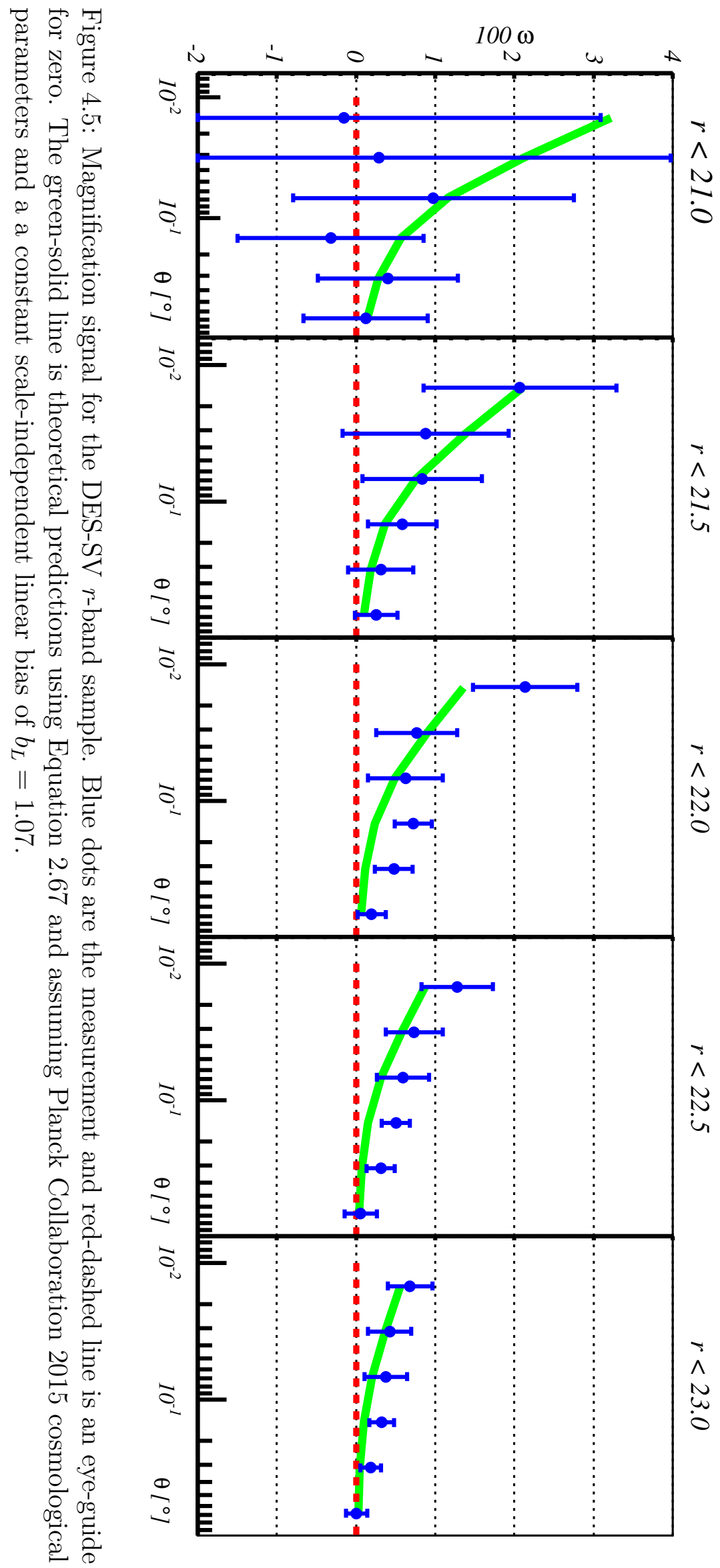




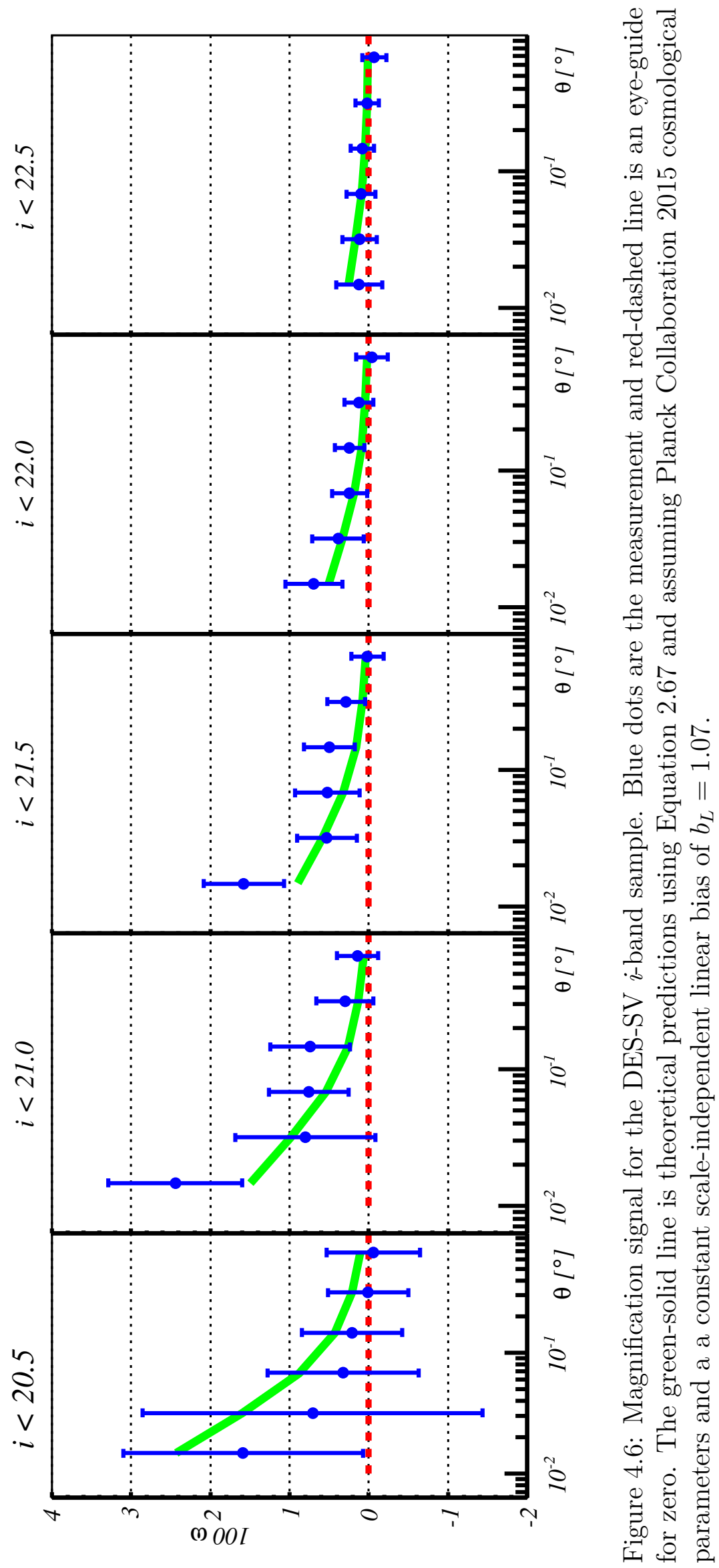




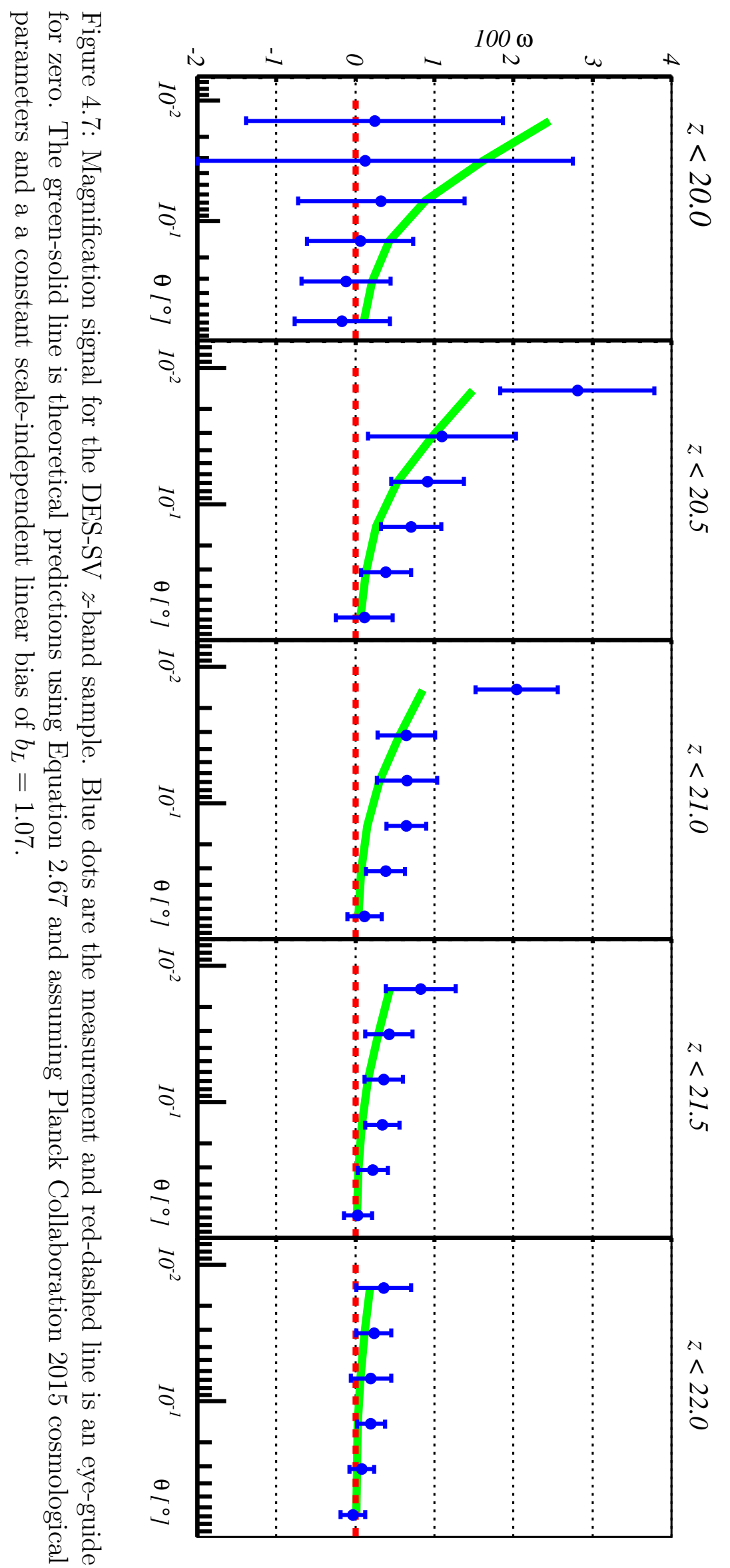




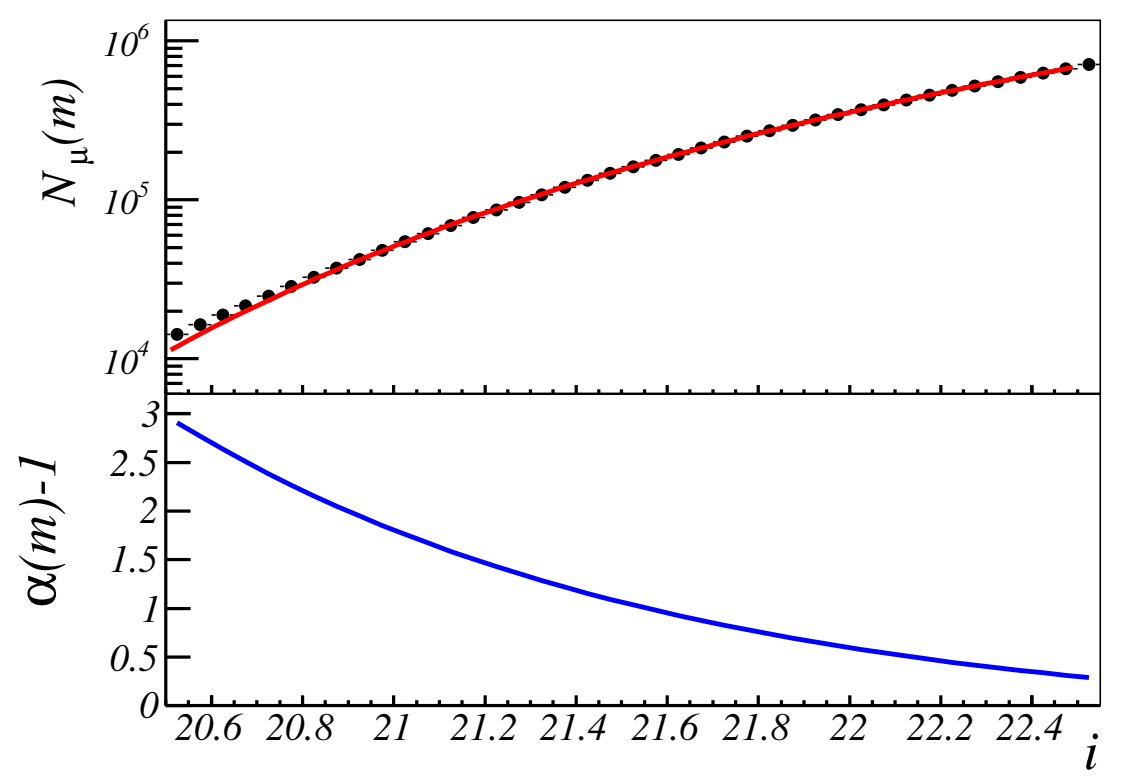

Figure 4.8: Top panel: Dots are the measured $i$-band cumulative number count as a function of the $i$-band magnitude. Red solid line is the fit using a Schechter function (see text). Bottom panel: number count slope $\alpha-1$ measured from the fitted Schechter function of the top panel.

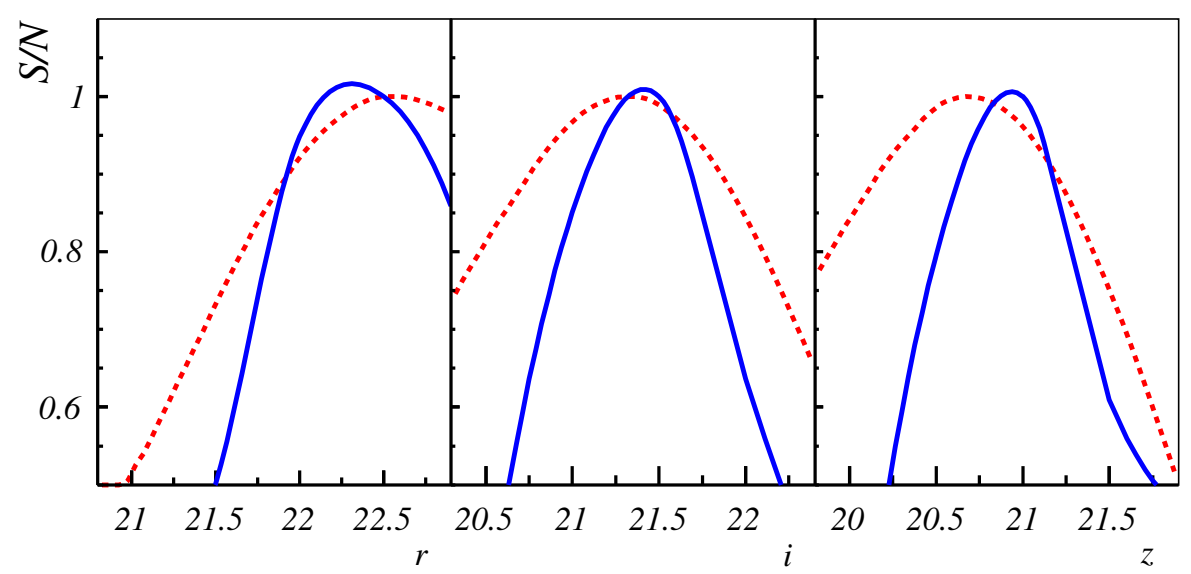

Figure 4.9: Red dashed line: expected signal-to-noise ratio computed with Equation 4.2. Blue solid line is the measured significance of the data. Both curves are normalized to their respective maximum. 
where $\tilde{\omega}, \omega$ are the measured and theoretical cross-correlation functions respectively. Goodness of fit tests are also made testing the hypothesis of absence of magnification:

$$
\begin{gathered}
\chi_{\text {zero }}^{2}= \\
\sum_{\eta \nu i j} \tilde{\omega}_{\mathrm{LS}_{\mathrm{i}}}\left(\theta_{\eta}\right) C^{-1}\left(\omega_{\mathrm{LS}_{\mathrm{i}}}\left(\theta_{\eta}\right) ; \omega_{\mathrm{LS}_{\mathrm{j}}}\left(\theta_{\nu}\right)\right) \tilde{\omega}_{\mathrm{LS}_{\mathrm{j}}}\left(\theta_{\nu}\right) .
\end{gathered}
$$

The $\chi^{2}$ values can be seen in Table 4.1 showing good agreement with the theoretical predictions described in chapter 2. To test which hypothesis is favored, the Bayes factor is used:

$$
\mathcal{B}=\frac{P(M \mid \Theta)}{P(Z \mid \Theta)}=\frac{P(\Theta \mid M)}{P(\Theta \mid Z)} \frac{P(M)}{P(Z)},
$$

where

$$
P(M \mid \Theta)=e^{-\chi_{\text {Planck }}^{2} / 2}
$$

and

$$
P(Z \mid \Theta)=e^{-\chi_{\mathrm{zero}}^{2} / 2}
$$

The assumed prior sets detection and non-detection of magnification to be equally probable: $P(M)=P(Z)$. Bayes factors are computed for each function individually as well as for each band using the full covariance.

The significance for each individual correlation function has a strong dependence on the considered magnitude limit of the sub-sample. At the bright cuts, shot-noise prevents the identification of a non-zero magnification signal. At the faint end, although the sub-samples are much more populated, the strength of the magnification signal is compatible with zero. This behaviour has been compared with the predictions (see section 4.1). Predicted and measured values are plotted together in Figure 4.9. It can be seen that the prediction of the location of the maximum signal-to-noise can only be used as a first approach.

To compute the significance of the detection for each band, the full covariance is used. One covariance matrix (see Figure 4.4 for the $i$-band matrix) per each band is computed taking into account the correlations between each magnitude cut. The logarithm of the Bayes factor can be found in Table 4.1, being all above 2, allowing to claim that magnification has been detected [165].

A usual approach to enhance the signal-to-noise ratio, is to define a unique source sample and weight each source galaxy with its corresponding $\alpha_{S}(m)-1$ value [97] and compute the two-point angular cross-correlation function. This weighting procedure is used at the samples $r<23.0, i<22.5$ and $z<22.0$. These correlation functions can be seen in Figure 4.10 with a comparison with the theoretical prediction and the correlation functions of the same sample computed without weighting. Significances of these measurement can be found in Table 4.1 with a marginal difference respect to the one computed without weighting using the five subsamples. 


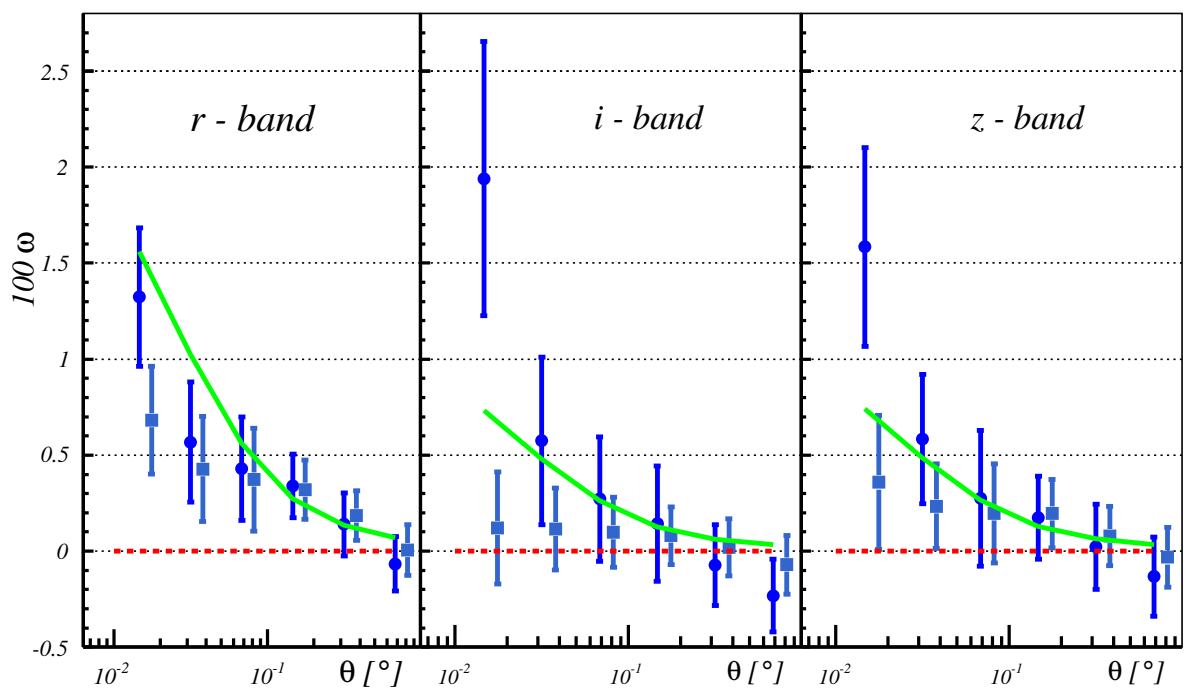

Figure 4.10: Measured two-point angular cross-correlation functions for the samples $r<23.0, i<22.5$ and $z<22.0$ left to right respectively. Dots use the optimal weighting [138], where each galaxy is weighted by its corresponding $\alpha_{S}(m)-1$ value, whereas squares are not weighted. Green line is the theoretical prediction. Red dashed line is an eye-guide for zero.

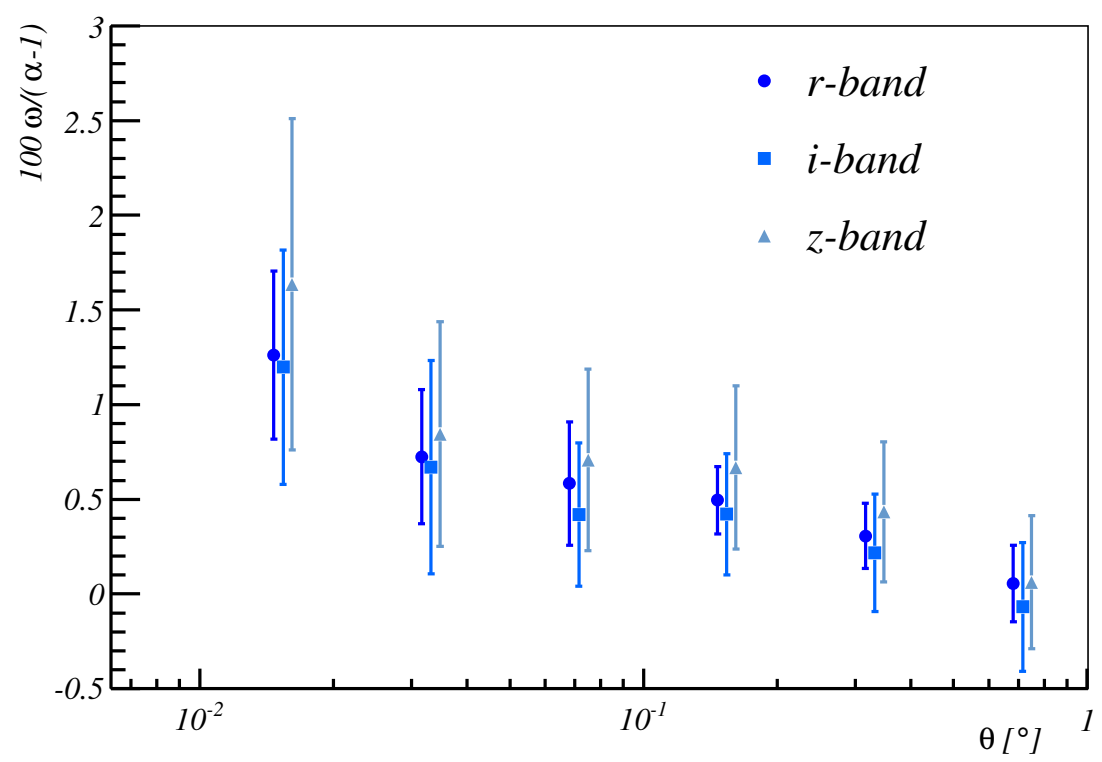

Figure 4.11: Example of the achromaticity of the measured signal. Here are shown the measured two-point angular cross-correlation functions for $r<22.5, i<22.0$ and $z<21.5$ divided by their corresponding $\alpha-1$. 
Finally, in order to test that the signal is achromatic, the measured two-point angular cross-correlation functions for each band, normalized by its $\alpha_{S}(m)-1$ are compared. All cross-correlation functions fluctuate within $1 \sigma$ errors (see Figure 4.11 for an example) demonstrating that the measured convergence field does not depend on the considered band.

\subsubsection{Systematic error analysis}

Here, the impact of potential sources of systematic errors on the measured twopoint angular cross-correlation function is investigated and how they are taken into account in the measurement is described.

\section{Number count slope $\alpha$}

When comparing the measured two point angular cross-correlation functions with the theoretical prediction via Equation 2.36 for a given set of cosmological parameters, $\alpha(m)$ is determined by fitting the cumulative number count distribution to Equation 4.9 and then using Equation 2.35. To compute the possible impact of the uncertainty of this fit on the comparison with theory, a marginalisation over all the parameters of fit $\left(A, m_{*}, \beta\right)$ is made.

Parameters are randomly sampled with a Gaussian distribution centerd on the value given by the fit to the cumulative number count and with a standard deviation equal to the $1 \sigma$ errors of the fit. The value of $\alpha$ is recalculated with these randomly sampled parameters. The impact of the dispersion of the $\alpha$ values obtained is negligible compared to the size of the jackknife errors, so they are not taken into account.

In addition to the parameter determination, a possible non-completeness on the SPT-E field can modify the magnitude distribution altering the cumulative number count slope parameter [142]. To estimate the possible impact of non-completeness, the measured magnitude distributions of the SPT-E field are compared with those of deeper fields measured by DES, such as the COSMOS field. Both distributions are found to be equal at the range of magnitudes considered on this analysis (see Figure 4.12 for an example in the $i$-band).

\section{Object obscuration}

Chang et al.[149] studied whether moderately bright objects in crowded environments produce a decrease in the detection probability of nearby fainter objects at scales $\theta \lesssim 10$ arcsec. However, such scales are well below those considered in this analysis $(\theta>36$ arcsec $)$ and therefore this effect is ignored.

\section{Stellar contamination}

For a given choice of star-galaxy classifier, there will be a number of stars misclassified as galaxies, so the observed two-point angular cross-correlation function $\omega_{O}(\theta)$ must be corrected by the presence of any fake signal induced by stars (see chapter A):

$$
\omega_{\mathrm{LS}_{\mathrm{j}}}=\frac{\omega_{\mathrm{O}}(\theta)-\lambda_{\mathrm{L}} \omega_{* \mathrm{~S}_{\mathrm{j}}}(\theta)-\lambda_{\mathrm{S}_{\mathrm{j}}} \omega_{\mathrm{L} *}(\theta)}{1-\lambda_{\mathrm{L}}-\lambda_{\mathrm{S}_{\mathrm{j}}}},
$$




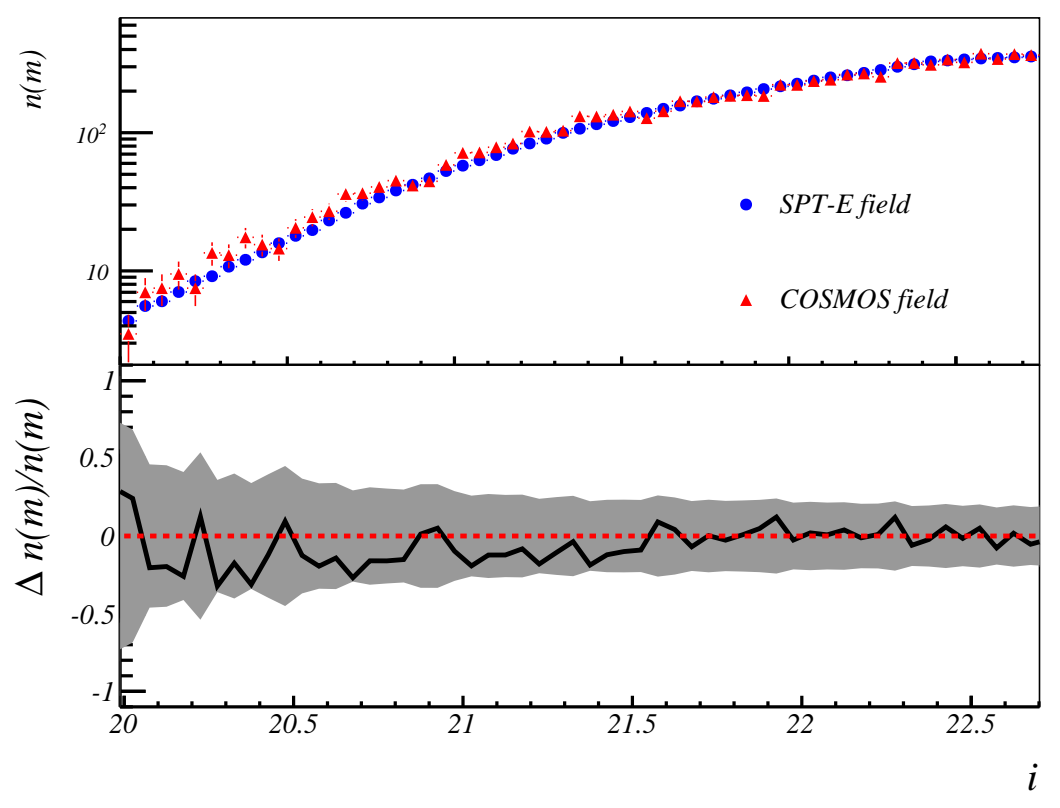

Figure 4.12: Upper panel: Comparison of the magnitude distribution for the SPT$\mathrm{E}$ and the COSMOS fields. Both histograms are normalized by their respective area. Lower panel: Relative difference between the magnitude distribution of the COSMOS and the SPT-E fields. The shaded region shows the $1 \sigma$ confidence interval computed from shot-noise.

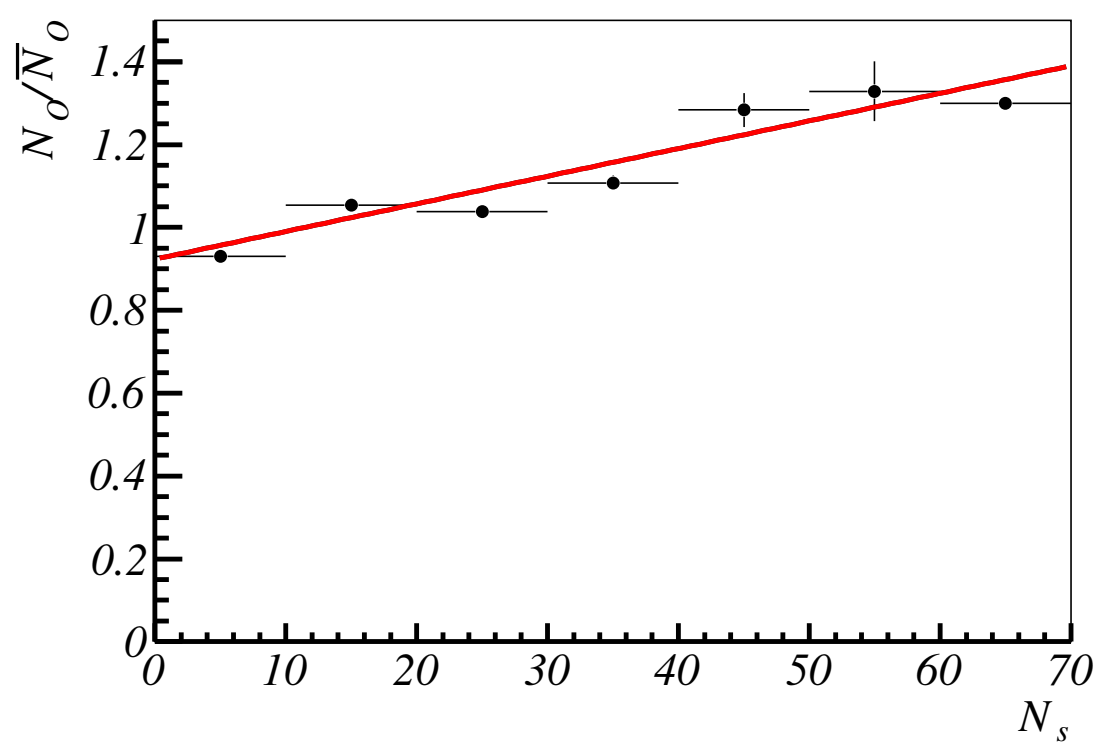

Figure 4.13: Determination of the purity of the lens sample. For each $N_{\text {nside }}=512$ HEALPIX-pixel, the number of objects classified as galaxies divided by the average number of galaxies per pixel is plotted as a function of the number of objects classified as stars. Black dots are the measured data. Red line is the linear fit to the data. The intercept of the line with the Y-axis is the estimated purity of the sample. 
where $\omega_{\mathrm{LS}_{\mathrm{j}}}$ is the corrected galaxy cross-correlation function, $\omega_{\mathrm{L} *}$ is the crosscorrelation function of the true galaxy lenses with the stars misclassified as galaxies in the source sample, $\omega_{* S_{j}}$ is the cross correlation of the stars misclassified as galaxies in the lenses with the true source galaxies and $\lambda_{\mathrm{L}}, \lambda_{\mathrm{S}_{\mathrm{j}}}$ are the fraction of stars in the lens and in the source samples respectively. Assuming that the misclassification of stars is spatially random and is a representative sample of the spatial distribution of the population classified as stars and that the fraction of misclassified stars is small, the functions $\omega_{\mathrm{L} *}, \omega_{* \mathrm{~S}_{\mathrm{j}}}$ are estimated from the cross-correlation of the galaxy population and the stellar population in the corresponding redshift bin.

Following a similar approach to [166], if the latter is true and the misclassified stars trace the global population of stars, for a given patch of the sky the number of objects classified as galaxies $N_{\mathrm{O}}$ must be the average number of true galaxies $\bar{N}_{\text {g }}$ plus a quantity proportional to the number of stars on that given pixel,

$$
N_{\mathrm{O}}=\bar{N}_{\mathrm{g}}+\tilde{\gamma} N_{\mathrm{s}}
$$

Dividing by the average number of objects marked as galaxies $\bar{N}_{\mathrm{O}}$,

$$
\frac{N_{\mathrm{O}}}{\bar{N}_{\mathrm{O}}}=p+\gamma N_{\mathrm{s}}
$$

where $p=\bar{N}_{\mathrm{g}} / \bar{N}_{\mathrm{O}}$ is the purity of the sample, that is, $\lambda=1-p$.

In order to estimate the purity of the galaxy sample with this method, an $N_{\text {side }}=512$ HEALPIX pixelation is made and for each pixel $N_{\mathrm{O}} / \bar{N}_{\mathrm{O}}$ and $N_{\mathrm{s}}$ is computed. Then, a fit to Equation 4.18 is made determining a purity of 94 per cent for the lens sample and about 98 per cent for the source sample depending on the considered band (see Figure 4.13 for an example). With this purity, the correction due to stellar contamination given by Equation 4.16 is found to be one order of magnitude smaller than the statistical errors (see Figure 4.14 for the $i$-band correction), so stellar contamination is not taken into account in the analysis. Nevertheless, on future analysis with more galaxies and area this may be important. Note that the objects labeled as stars by our star-galaxy classifier would be a combination of stars and galaxies thus these calculations are an upper bound to stellar contamination.

\section{Survey observing conditions}

Observing conditions are not constant during the survey, leading to spatial dependencies across the DES-SV footprint [167] that may affect the observed crosscorrelation function, such as seeing variations, air-mass, sky-brightness or exposure time [141]. To trace these spatial variations, the catalog produced by the Monte Carlo sampling code BALRoG has been used as random sample [160]. It is important to remark that BALROG catalogs are produced with the same pipeline as DES-SV data, allowing one to trace subtle effects such as patchiness on the zeropoints, deblending and possible magnitude errors due to a wrong sky subtraction close to bright objects. 


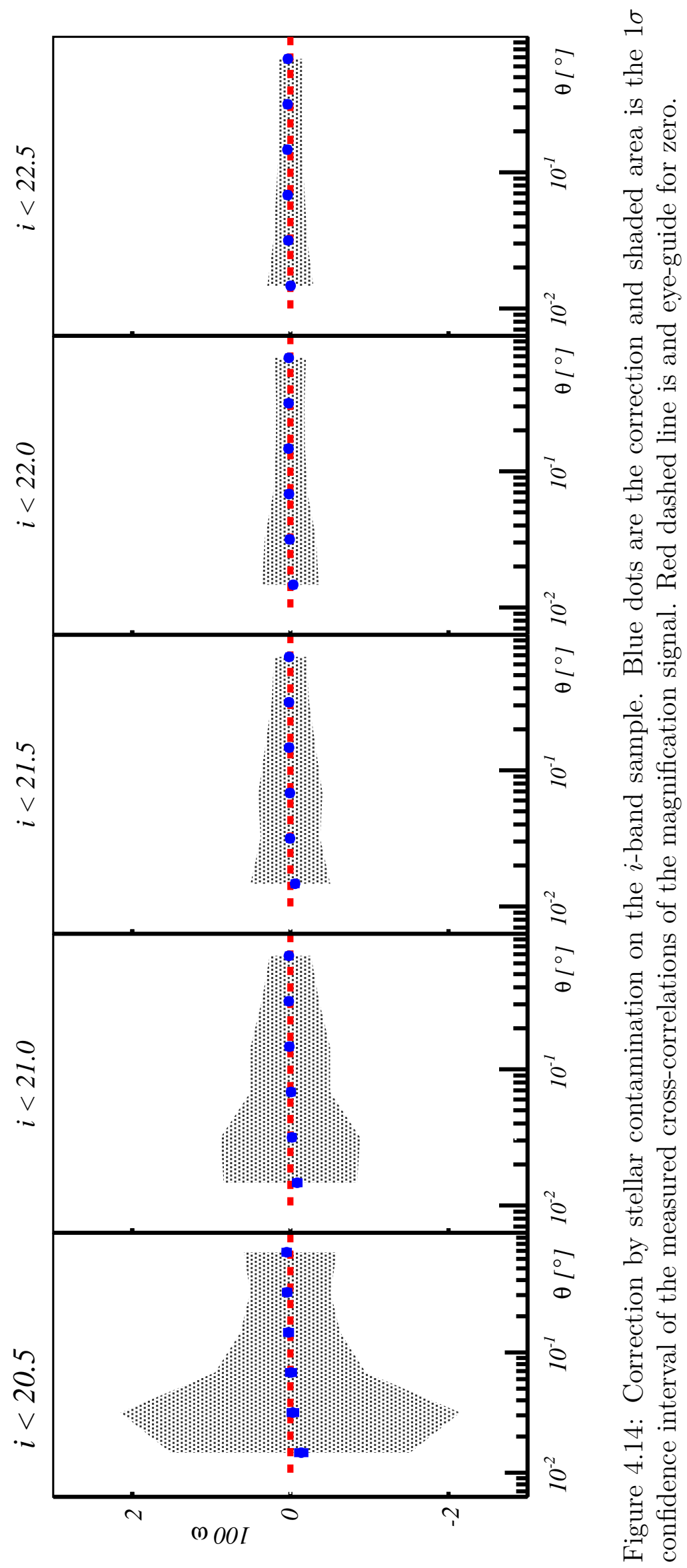


The BALROG catalogs are DES-like catalogs, where no intrinsic magnification signal has been included. The BALROG software generates images of fake objects, all with zero convergence $\kappa$, that are embedded into the DES-SV coadd images (convolving the objects with the measured point spread function, and applying the measured photometric calibration). Then SExTRACTOR was run on them, using the same DES Data Management configuration parameters used for the image processing. The positions for the simulated objects were generated randomly over the celestial sphere, meaning that these positions are intrinsically unclustered. Hence, the detected BALROG objects amount to a set of random points, which sample the survey detection probability. For a full description and an application to the same measurement as in [148] see [160]. This is the first time that this extensive simulation is used to correct for systematics. The same cuts and masking of the data sample (subsection 4.3.1) are also applied to the the BALROG sample. A re-weighting following a nearest-neighbours approach was applied to BALROG objects in order to follow the same magnitude distribution of the DES-SV data on both lens and sources.

The use of Monte Carlo sampling methods provides a new approach to mitigate systematic effects complementary to methods that cross-correlate the galaxypositions with the maps of the survey observing conditions [141, 166, 168] or involve masking the regions of the sky with worst values of the observing conditions [148]. The amount of sky to be masked in order to mitigate the systematic effects on the correlation functions, is decided based on the impact on the correlation function, which may lead to a biassed measurement. On the other hand, the approach involving cross-correlations may lead to an overcorrection effect since the different maps of the observing conditions are, in general, correlated in a complicated manner [169]. This new Monte Carlo technique to sample the selection function of the survey given by BALROG, has the advantage that takes into account the correlation of the different observing conditions maps as well as provides an objective criteria to mitigate systematic errors on the correlation function for a given sample, avoiding biassed measurements. In addition, the use of BALROG has the potential to allow us in the future to exploit the full depth of the survey [160].

BALROG allows to explore how the real properties of the galaxies are mapped into the observed ones. One might think that it may be translated from observed to real quantities. This is not possible since that although the real $\rightarrow$ observed map is one-to-one, meanwhile the observed $\rightarrow$ real is not.

Although the use of BALROG is a ground-breaking technique on the mitigation of systematic errors, its limitation must be taken into account. The simulated catalogs inherit the properties of the input catalogs. Thus, if an specific population of galaxies is not accurately described in the input, it will not be accurately taken into account. DES-SV BALROG catalogs are based on the COSMOS survey ${ }^{8}$, that is based on images from the Hubble Telescope which are deeper than the

\footnotetext{
${ }^{8}$ cosmos.astro.caltech.edu
} 
DES survey. Thus, the overall quality of the input catalog should be good enough to simulate DES and this should not be a limitation on this analysis.

\section{Dust extinction}

The possible presence of dust in the lenses may modify the observed magnitude in addition to the magnitude shift due to magnification [95]. The change in magnitude $(\delta m)$ on the $p$-band may be written as

$$
\delta m_{p}=-2.5 \log \mu+\frac{2.5}{\ln 10} \tau_{p},
$$

where $\mu \simeq 1+2 \kappa$ is the change in magnitude due to magnification and $\tau_{k}$ is the optical depth due to dust extinction. Whereas magnification is achromatic, dust extinction induces a band-dependent magnitude change. Taking this into account, the color-excess for bands $p, q^{9}$ is defined as

$$
E_{p q}=\delta m_{p}-\delta m_{q}=1.08\left[\tau_{p}-\tau_{q}\right] .
$$

Define the color-density cross-correlation as [95]

$$
\left\langle\delta_{\mathrm{g}} E_{p q}\right\rangle(\theta)=1.09\left[\tau_{p}(\theta)-\tau_{q}(\theta)\right],
$$

where $\delta_{\mathrm{g}}$ is the density contrast of the lenses and $E_{p q}$ is the color-excess of the sources; from the measurements by [95] it can be parametrized as

$$
\left\langle\delta_{\mathrm{g}} E_{p q}\right\rangle(\theta)=1.09 \tau_{V}\left[\frac{\lambda_{V}}{\lambda_{p}}-\frac{\lambda_{V}}{\lambda_{q}}\right]\left(\frac{\theta}{1^{\prime}}\right)^{-0.8},
$$

with $\tau_{V}=2.3 \times 10^{-3}$ the optical depth at the $V$-band and $\lambda_{V}, \lambda_{p}, \lambda_{q}$ the average wavelengths of the $V, p$ and $q$ bands respectively. With this parametrization, the impact of dust extinction is negligible at the scales considered on this analysis. As it can be seen in Figure 4.15, color-density cross-correlation functions are compatible with Equation 4.22 as well as with zero.

In addition, the impact of a dust profile has been simulated as described in Equation 4.22 with the MICE simulation (section 4.2). To do so, for each galaxy belonging to the source sample a magnitude shift is induced

$$
m_{d}=m_{\mu}+1.09 \tau_{V} \frac{\lambda_{V}}{\lambda} \sum_{l}\left(\frac{\theta_{l}}{1^{\prime}}\right)^{-0.8} .
$$

Here $\theta_{l}$ is the angular separation of the source-galaxy and the $l$-th lens galaxy and the summation is over all the galaxies of the lens sample. In Figure 4.16 the difference between the two-point angular cross-correlation with and without the dust can be seen to be less than the statistical errors. It can be deduced that dust has no impact on the angular scales considered on this work.

Since the parametrization used here only applies to a sample similar to the one used at [95], statements about dust constrains are limited. Nevertheless this does not change the fact that no chromatic effects are detected.

\footnotetext{
${ }^{9}$ In this section $p, q$ stand for a generic index label while $V$ stands for the $V$ band of the $U B V$ system.
} 


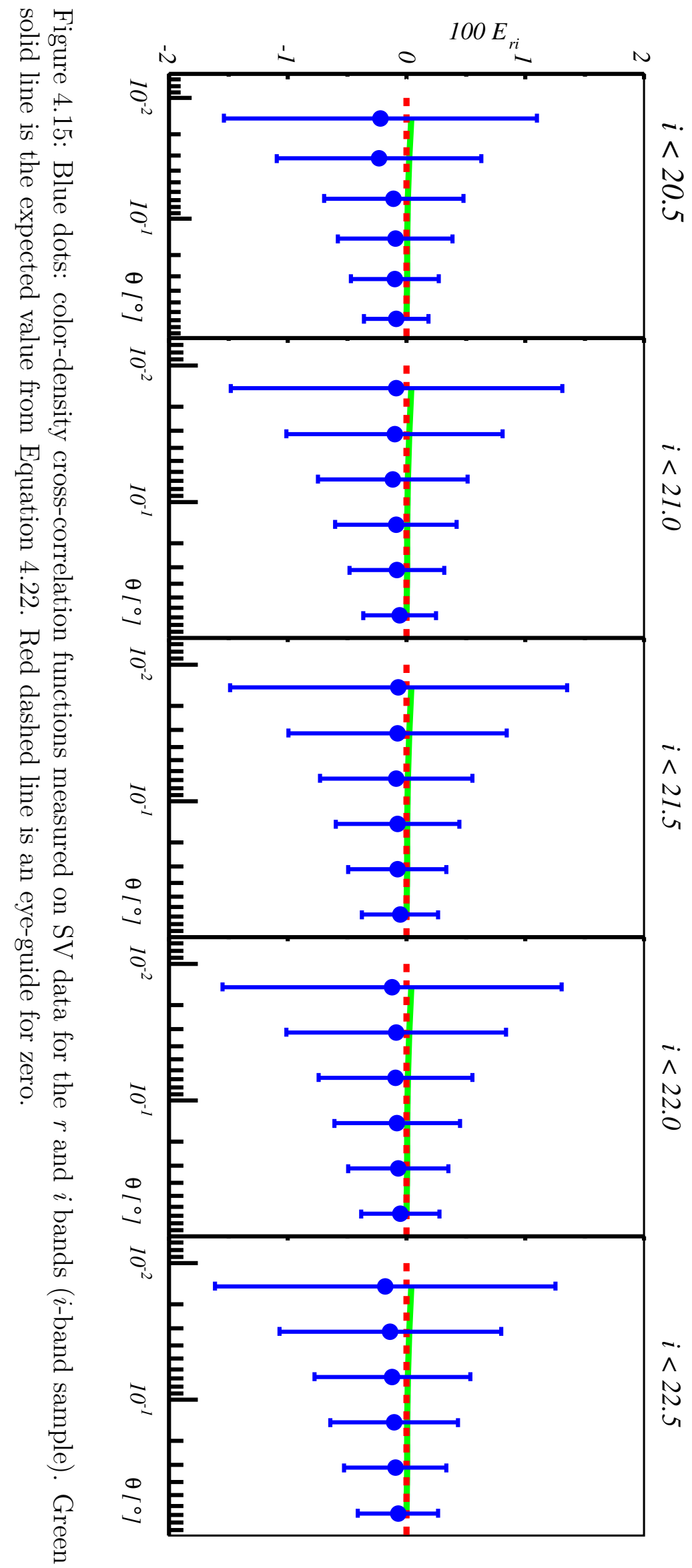




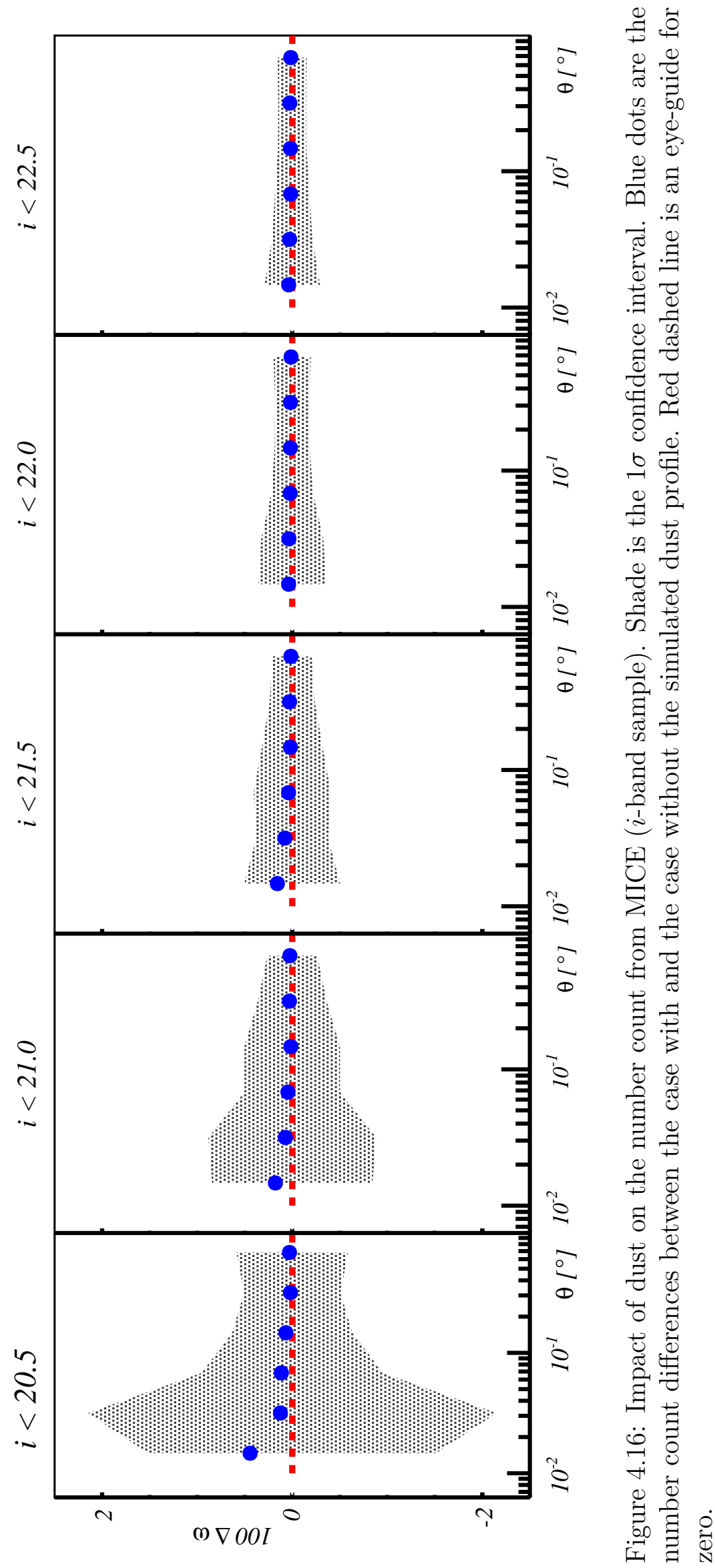




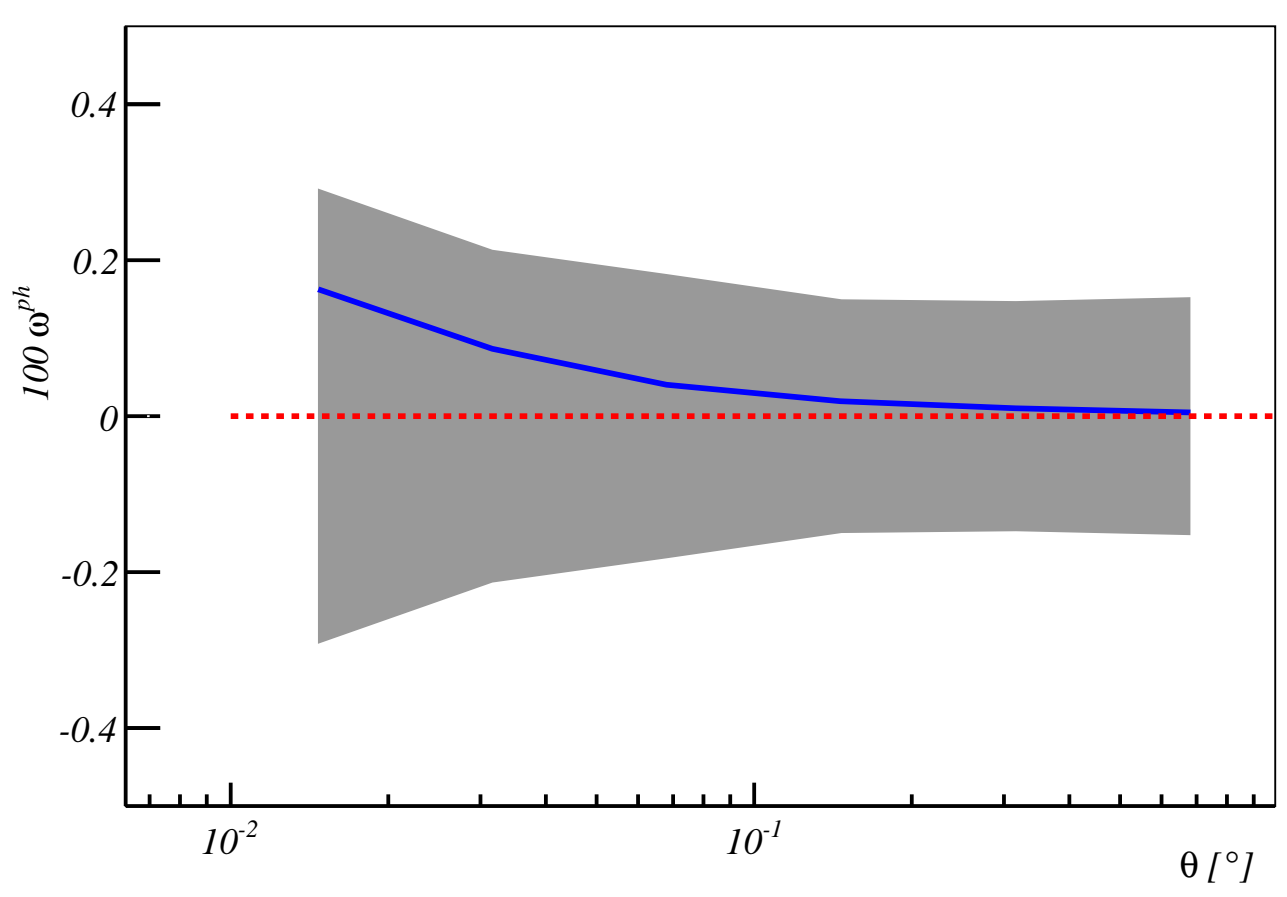

Figure 4.17: Comparison of $1 \sigma$ jackknife errors of the measured correlation function (grey shade) with the expected signal induced by the photo-z migration between the lens and the source sample (sample $i<22.5$ ) computed theoretically with the stacking of the pdfs (blue line).

\section{Photometric redshifts}

A general study of photo-z performance in DES-SV can be found in [157]. A comprehensive study of the photo-z performance and its implications for weak lensing for this data can be found in [170]. Both studies are followed in this analysis.

Conservative photo-z cuts are made in order to minimize migration between lens and source samples. Nevertheless, catastrophic outliers in the photo-z determination can bias the measurement of $\kappa$ [171]. Thus, the tails of the probability density functions (pdfs) of the photo-z code are a crucial systematic to test.

As mentioned in chapter 2 , in addition to the magnification signal, galaxy migration due to a wrong photo-z assignment between lens and source samples may induce a non-zero cross-correlation signal due to the physical signal coming from the clustering of objects in the same redshift bin. As a first approach, estimation of the expected signal induced by photo-z migration $\left(\omega^{p h}\right)$ is computed with Equation 2.28:

$$
\omega_{\mathrm{LS}_{\mathrm{j}}}^{\mathrm{ph}}(\theta)=\int_{0}^{\infty} d z \int_{0}^{\infty} d z^{\prime} \phi_{\mathrm{L}}(z) \phi_{\mathrm{S}_{\mathrm{j}}}\left(z^{\prime}\right) \xi\left(\theta ; z, z^{\prime}\right),
$$

where $\xi\left(\theta ; z, z^{\prime}\right)$ is the $3 \mathrm{D}$ correlation-function and $\phi_{\mathrm{L}}, \phi_{\mathrm{S}_{\mathrm{j}}}$ are the redshift distribution of the lens $(\mathrm{L})$ sample and the source sample $\left(\mathrm{S}_{\mathrm{j}}\right)$ estimated from the stacking 
of the pdfs given by TPZ. Figure 4.17 compares the measured two-point angular cross-correlation and the expected signal induced by photo-z can be seen for the $I$ sample. The signal induced by photo-z is found to be smaller than the statistical errors. Note that this method relies on an assumed cosmology and bias model, and therefore should be considered only an approximation. A more accurate calculation can be made with the help of N-body simulations.

From the overlap of the redshift distribution of both lens and source samples, it is found that the total photo-z migration between lens and source sample is $o \sim 0.6 \%$ depending on the magnitude cut of the source sample. The procedure to compute this overlap is to integrate the product of the pdfs of the lens and source sample:

$$
o=\int_{0}^{\infty} d z \phi_{L}(z) \phi_{S}(z)
$$

where $\phi_{L}, \phi_{S}$ are the stacked pdfs of the lens and source sample respectively. Since TPZ provides an individual pdf for each galaxy, the stacked pdf of a given sample is computed by adding all the individual pdfs of the galaxies that belong to that sample (see [172] for a study of clustering with stacked pdfs).

To estimate the maximum photo-z migration allowed between the lens and the source sample, the MICE simulation (section 4.2) with the un-lensed coordinates and magnitudes is used. Galaxies are randomly sampled on the lens redshift bin and then placed on the source redshift bin. Conversely, galaxies on the source redshift bin are randomly sampled and placed on the lens redshift bin. For a given lens or source sample, the number of galaxies introduced from the other redshift bin is chosen to be $0.1,0.3,0.5,0.7,0.9$ and 2 per cent of the galaxies. Then, the two-point angular cross-correlation is computed for each case. The difference of the correlation functions measured at the simulation with induced migration between lens and source sample and the original used in section 4.2 is the signal induced by photo-z migration. The signal induced by photo-z for the cases with 0.9 and 2 per cent computed with this method can be seen at Figure 4.18. It is found that at 0.9 per cent of contamination, the induced signal due to photo-z migration is comparable to the error in the correlation functions. This upper limit is greater than the estimated photo-z migration, demonstrating that the effect of photo-z migration is negligible. Photo-z migration has a larger impact on the brightest samples. Nevertheless, since the errors of the correlation functions of these samples are shot-noise dominated, the tightest constrains on photo-z migration are imposed by the faintest samples. With a larger data sample this statement will no longer be true.

Photo-z induced correlation functions that mimic magnification may affect the measured significance. Thus, Bayes factor is recomputed with two new hypothesis, the measured signal is a combination of magnification and photo-z $(M+P h)$ or 


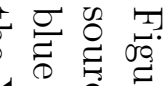
$x \div \frac{0}{0}$

. A $\begin{array}{ccc}0 \\ \text { in }\end{array}$

क्ष

.

क 它

ڤ

总宫

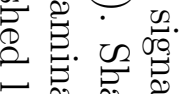

F.

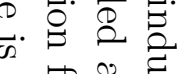

๑

过 范

象菑范

○े

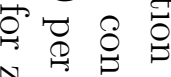

\&㟒吊

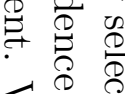

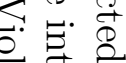

क

纪

ऐ)

ᄋ 客官

范

总总

C.

汽

๑)

尺̊

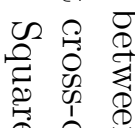

क \&

๑

䜦. 苍.

एँ

尺 $\bullet$

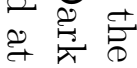

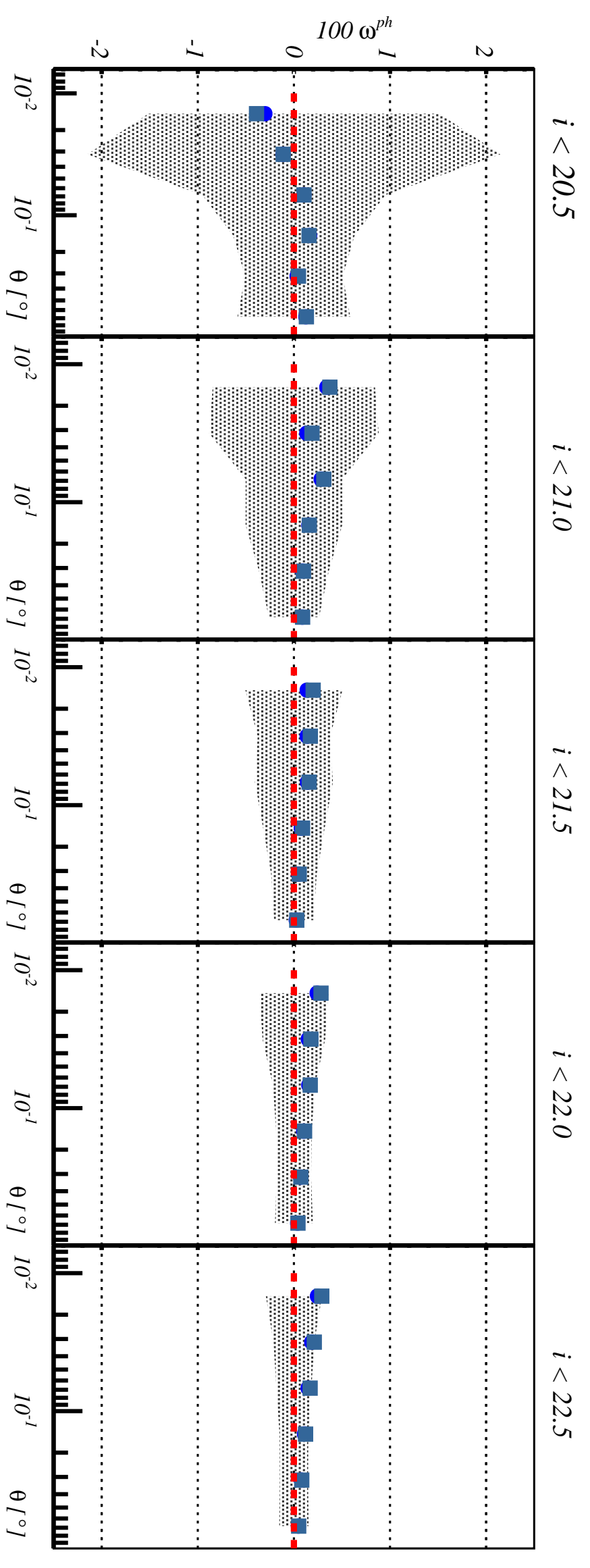


the measured signal is only photo-z $(P h)$ :

$$
\mathcal{B}=\frac{P(M+P h \mid \Theta)}{P(P h \mid \Theta)}=\frac{P(\Theta \mid M+P h)}{P(\Theta \mid P h)},
$$

where

$$
P(\Theta \mid M+P h)=e^{-\chi_{\text {Planck }+ \text { Ph }}^{2} / 2}
$$

and

$$
P(\Theta \mid P h)=e^{-\chi_{\mathrm{Ph}}^{2} / 2} .
$$

To compute $\chi_{\mathrm{Planck}+\mathrm{Ph}}^{2}$ and $\chi_{\mathrm{Ph}}^{2}$ it has been assumed that the expected theory is given by $\omega_{\mathrm{LS}_{\mathrm{j}}}(\theta)+\omega_{\mathrm{LS}_{\mathrm{j}}}^{\mathrm{ph}}(\theta)$ and $\omega_{\mathrm{LS}_{\mathrm{j}}}^{\mathrm{ph}}$ respectively, where $\omega_{\mathrm{LS}_{\mathrm{j}}}^{\mathrm{ph}}$ is the expected signal induced by photo-z computed using Equation 4.24. The significances recomputed using these two new hypothesis for the $r, i$ and $z$ bands are $\log _{10} \mathcal{B}=2.5,4.0,3.5$ respectively. Thus, it can be concluded that photo-z migration has a limited impact on the measured significances.

All previous calculations were based on the assumption that the pdfs are a reliable description of the true redshift distribution. This statement can be partially validated comparing the pdfs with the spectroscopic redshift distribution for the same sample (see Figure 4.19 for an example). Redshift distributions predicted by $\mathrm{TPZ}$ are found to be representative of those given by the spectroscopic sample. Nevertheless, this statement has limitations -but is good enough for SV data- and a more accurate description of the real redshift distribution of the full sample will be measured with methodologies involving clustering-based estimators [173-176] when the size of the data sample grows. This type of estimators involve the use of two-point angular cross-correlations between different redshift bins, whose measurement may be biassed by number count magnification itself. Nevertheless, as it has been stated in chapter 2, depending on the value of the number count slope, the amplitude induced by magnification on the correlation-function may be zero. Thus, when employing this kind of estimators, samples should be carefully chosen so that $\alpha_{S}-1=0$. This can be done by measuring the number count slope at the cumulative magnitude distribution with methods such that used in this work.

Finally, to demonstrate that the measured signal is independent of the photo-z technique employed to estimate the redshift, the two-point angular cross-correlation functions used on this analysis are re-computed with redshift estimated with other two different approaches that have shown to have similar performance as TPZ [157] a neural network, Skynet [177], and a template based approach, Bayesian Photo-Z (BPZ) [178]. Figure 4.20 compares the cross-correlations computed with the three codes for the $i$-band, showing them to be within $1 \sigma$ errors.

\subsubsection{Discussion}

On this analysis, the weak-lensing magnification signal has been detected on the DES-SV data using the general population of galaxies. In addition, a thorough and detailed study of the systematic effects has been made. The systematic error 


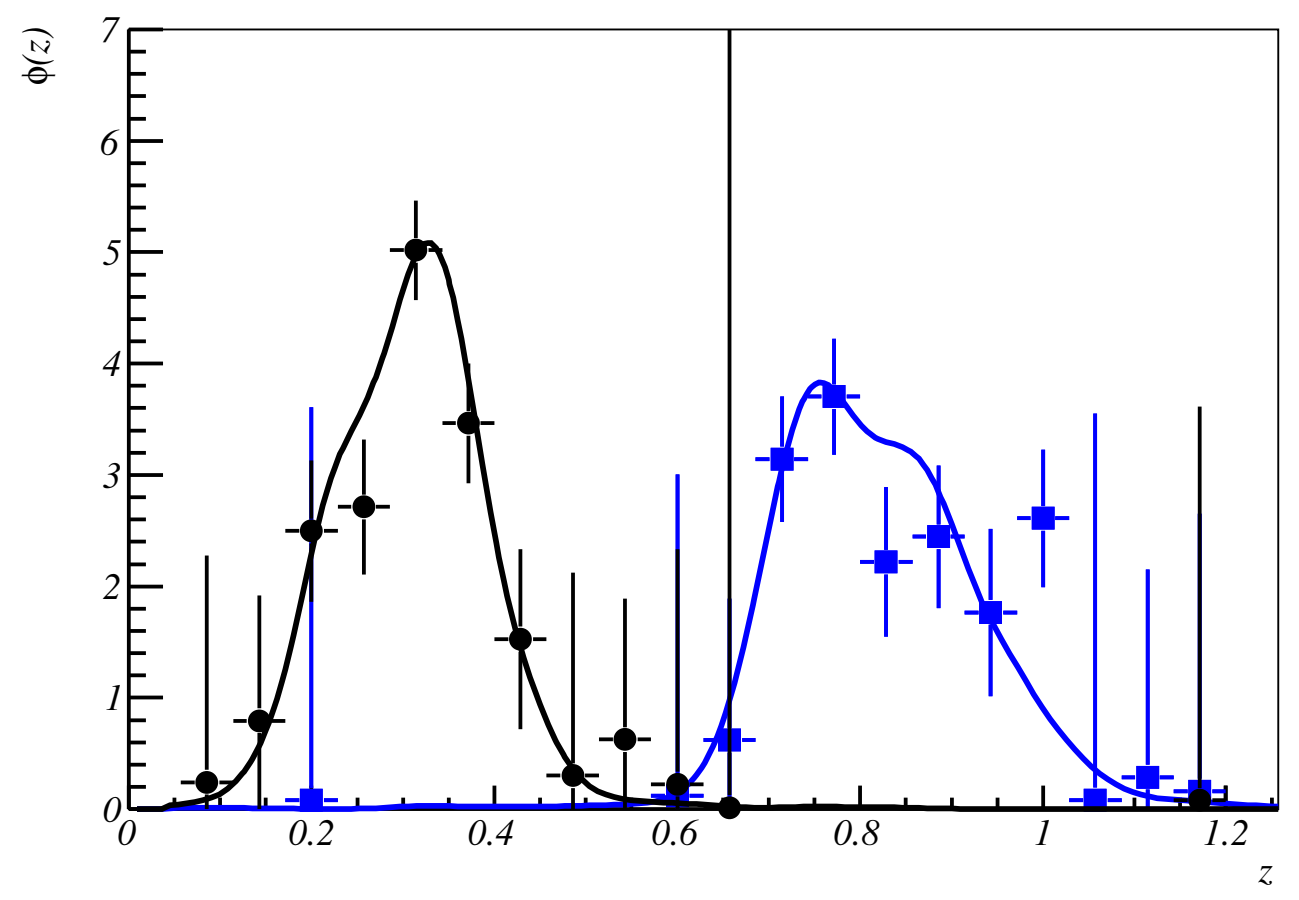

Figure 4.19: Comparison of the redshift distribution computed by the stacking of the pdfs given by TPZ ( solid lines) with the ones computed with the spectroscopic sample of the lens (black dots) and the source sample $i<22.5$ (blue squares). 


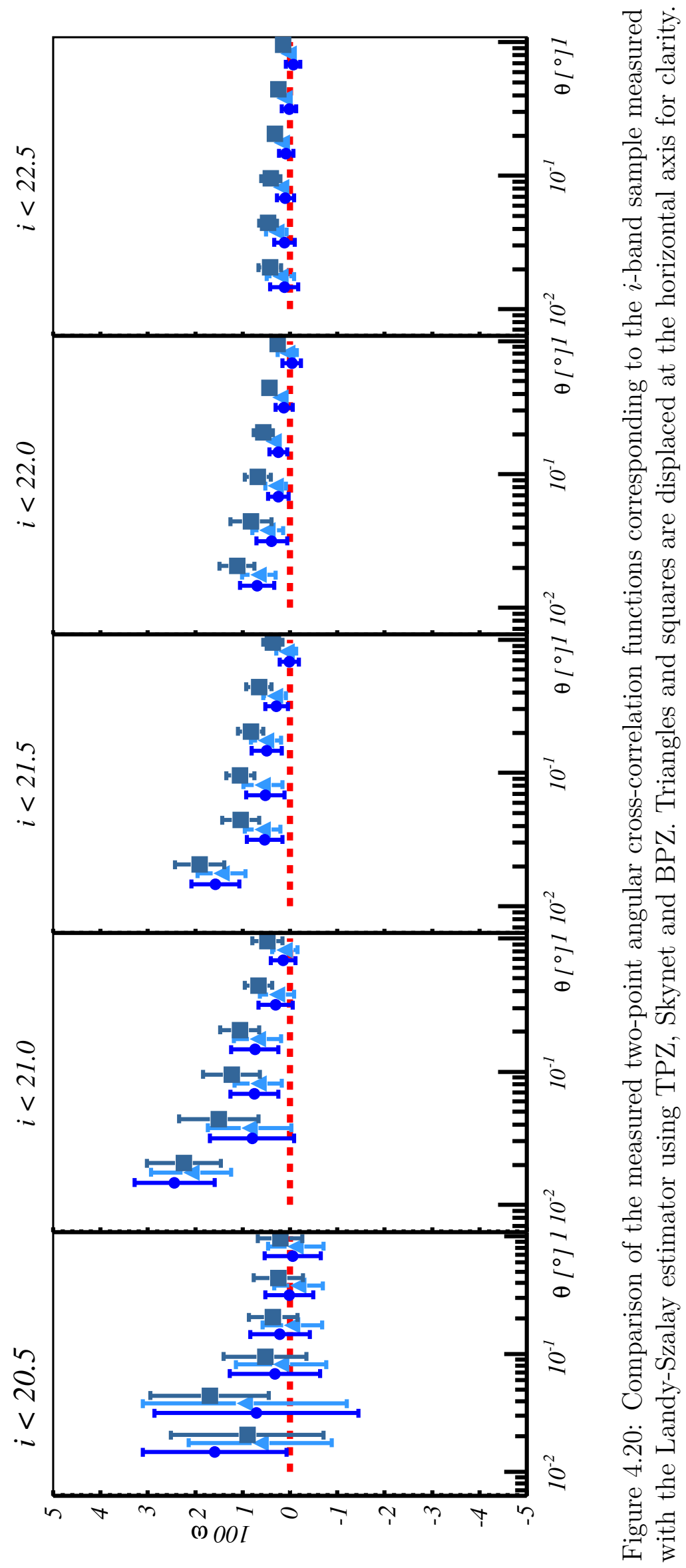





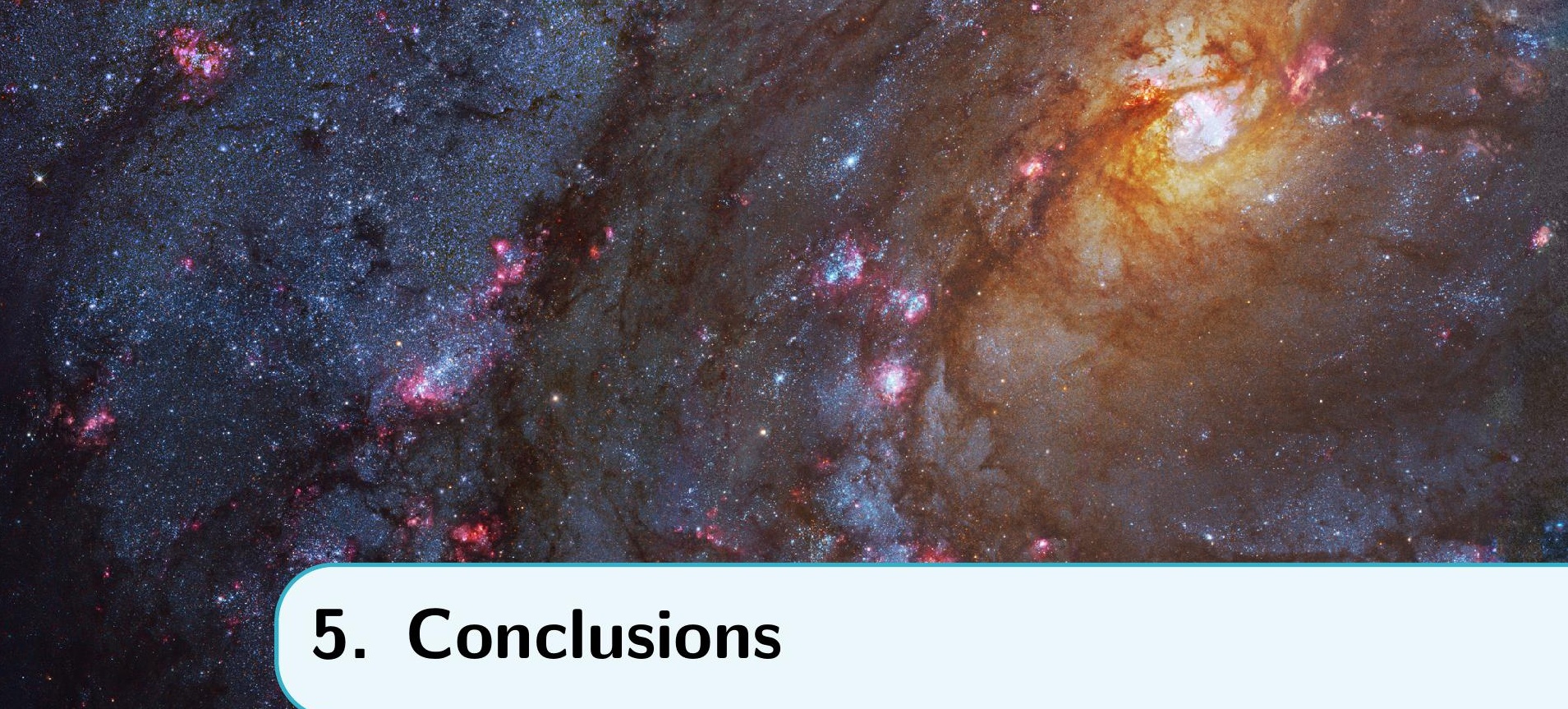

In this Thesis, the weak-lensing magnification has been measured using two datasets for the Dark Energy Survey: the Science Verification (DES-SV) and the Year 1 (DES-Y1), with different goals each. The DES-SV analysis developed a methodology to measure and mitigate the impact of systematic errors on number count magnification with wide-field photometric surveys. On the other hand, the DES-Y1 analysis used the techniques employed at DES-SV to measure the convergence profile of voids and trough to provide constrains on cosmology.

The nature of dark energy -the responsible of the accelerated expansion of the Universe-, constitutes one of the biggest puzzles of Modern Cosmology. The emptiest regions of the Universe -voids and troughs- are dominated by dark energy. Thus, its structure and evolution constitutes a powerful probe to shed light on dark energy. One of the physical observables from voids and troughs that is sensitive to dark energy is their convergence profile, that is a direct proxy for the matter profile. Due to the presence of dark matter, the matter profile is only directly accessible with gravitational lensing, since the other probes require the parametrization on how galaxies assembly within dark matter.

Weak-lensing magnification provides a direct measurement of the convergence profile. There are three observables that allow the determination of the convergence profile: number count magnification, size magnification and flux/magnitude magnification. These three methodologies can be combined to provide more accurate measurements and mitigate systematic errors. In addition, magnification can be combined with gg-lensing measurements to improve the systematic error correction since the gg-lensing also probes the convergence profile, but the sources of systematic errors are different.

In this work, a methodology to correct the effect of systematic errors using two kinds of simulations (N-body and image simulations) has been developed. 
The most important systematic errors to be taken into account are the observing conditions of the survey and the photo-z overlap of the lenses and sources. To overcome the survey observing conditions, the BALROG image-simulation has been used for the first time. The use of BALROG has demonstrated to provide reliable and unbiased corrections for these kind of systematic errors. In addition, this simulation maps depth inhomogeneities, allowing to use the full depth of the survey, increasing the area and number of available galaxies. On the other hand, the impact photo-z overlap was estimated with the help of the MICE N-body simulation and then hard photo-z cuts where made requiring a negligible impact on the magnification signal.

Finally, on this work the convergence profile of voids an troughs has been measured for the first time using number count magnification. This measurement opens the window to a new class of cosmological probes, since weak-lensing magnification by voids and troughs constitutes a new and independent probe for dark energy that on the immediate future may provide competitive and reliable cosmological constrains. 


\section{A. Stellar contamination equation}

The observed density contrast of objects is given by

$$
\delta_{\mathrm{O}}\left(\hat{\boldsymbol{n}}, z_{i}\right)=\frac{N_{\mathrm{g}}\left(z_{i}\right)+N_{*}\left(z_{i}\right)}{\bar{N}_{\mathrm{g}}\left(z_{i}\right)+\bar{N}_{*}\left(z_{i}\right)}-1,
$$

where $N_{\mathrm{g}}, N_{*}$ are the number of galaxies on direction $\hat{\boldsymbol{n}}$ and redshift $z_{i}$ and stars respectively and $\bar{N}_{\mathrm{g}}, \bar{N}_{*}$ the average number of galaxies and stars over the footprint. The previous equation can be expressed as

$$
\delta_{\mathrm{O}}\left(\hat{\boldsymbol{n}}, z_{i}\right)=\frac{N_{\mathrm{g}}\left(z_{i}\right)+N_{*}\left(z_{i}\right)}{\bar{N}_{\mathrm{g}}\left(z_{i}\right)\left[1+\frac{\bar{N}_{*}\left(z_{i}\right)}{\bar{N}_{\mathrm{g}}\left(z_{i}\right)}\right]}-1 .
$$

Taylor expanding the brackets one has,

$$
\delta_{\mathrm{O}}\left(\hat{\boldsymbol{n}}, z_{i}\right)=\frac{N_{\mathrm{g}}\left(z_{i}\right)+N_{*}\left(z_{i}\right)}{\bar{N}_{\mathrm{g}}\left(z_{i}\right)}\left[1-\frac{\bar{N}_{*}\left(z_{i}\right)}{\bar{N}_{\mathrm{g}}\left(z_{i}\right)}\right]-1
$$

and taking common factor $\bar{N}_{*}\left(z_{i}\right) / \bar{N}_{\mathrm{g}}\left(z_{i}\right)$,

$$
\begin{gathered}
\delta_{\mathrm{O}}\left(z_{i}\right)=\left[\frac{N_{\mathrm{g}}\left(z_{i}\right)}{\bar{N}_{\mathrm{g}}\left(z_{i}\right)}-1\right]+ \\
\frac{\bar{N}_{*}\left(z_{i}\right)}{\bar{N}_{\mathrm{g}}\left(z_{i}\right)}\left[\frac{N_{*}\left(z_{i}\right)}{N_{*}\left(z_{i}\right)}-\frac{N_{\mathrm{g}}\left(z_{i}\right)}{N_{\mathrm{g}}\left(z_{i}\right)}\right]-\frac{N_{*}\left(z_{i}\right)}{\bar{N}_{\mathrm{g}}\left(z_{i}\right)} .
\end{gathered}
$$

Assuming that $\bar{N}_{*} \ll \bar{N}_{g}$, the last term can be neglected and defining $\lambda_{i}=$ $\bar{N}_{*}\left(z_{i}\right) / \bar{N}_{\mathrm{g}}\left(z_{i}\right)$ as the fraction of stars on the $i$-th sample,

$$
\delta_{\mathrm{O}}\left(\hat{\boldsymbol{n}}, z_{i}\right)=\delta_{\mathrm{g}}\left(\hat{\boldsymbol{n}}, z_{i}\right)+\lambda_{i}\left[\delta_{*}\left(\hat{\boldsymbol{n}}, z_{i}\right)-\delta_{\mathrm{g}}\left(\hat{\boldsymbol{n}}, z_{i}\right)\right] .
$$

Calculating the two point angular cross-correlation results finally in

$$
\omega_{\mathrm{O}}=\left(1-\lambda_{i}-\lambda_{j}\right) \omega_{\mathrm{gg}}+\lambda_{j} \omega_{\mathrm{g} *}+\lambda_{i} \omega_{* \mathrm{~g}}+\lambda_{i} \lambda_{j} \omega_{* *} .
$$





\section{B. Additional figures}

Here the plots and graphs not included on the main text are included. 


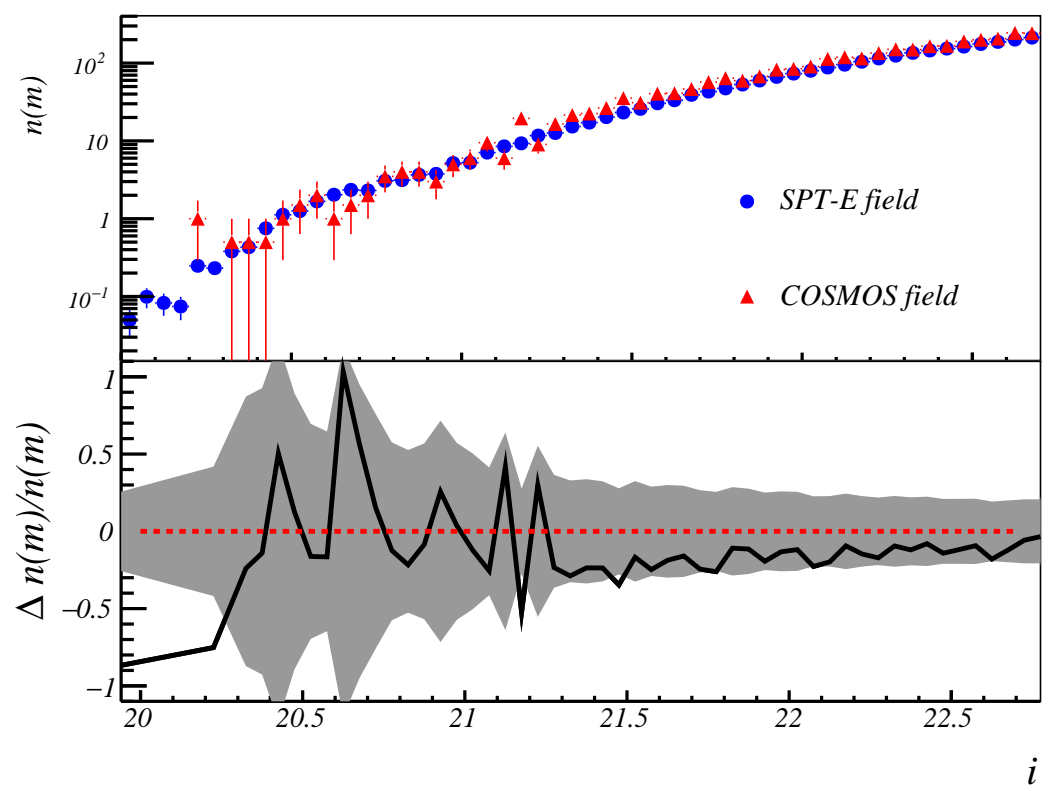

Figure B.1: Upper panel: Comparison of the magnitude distribution for the SPT-E and the COSMOS fields for the $r$ band.

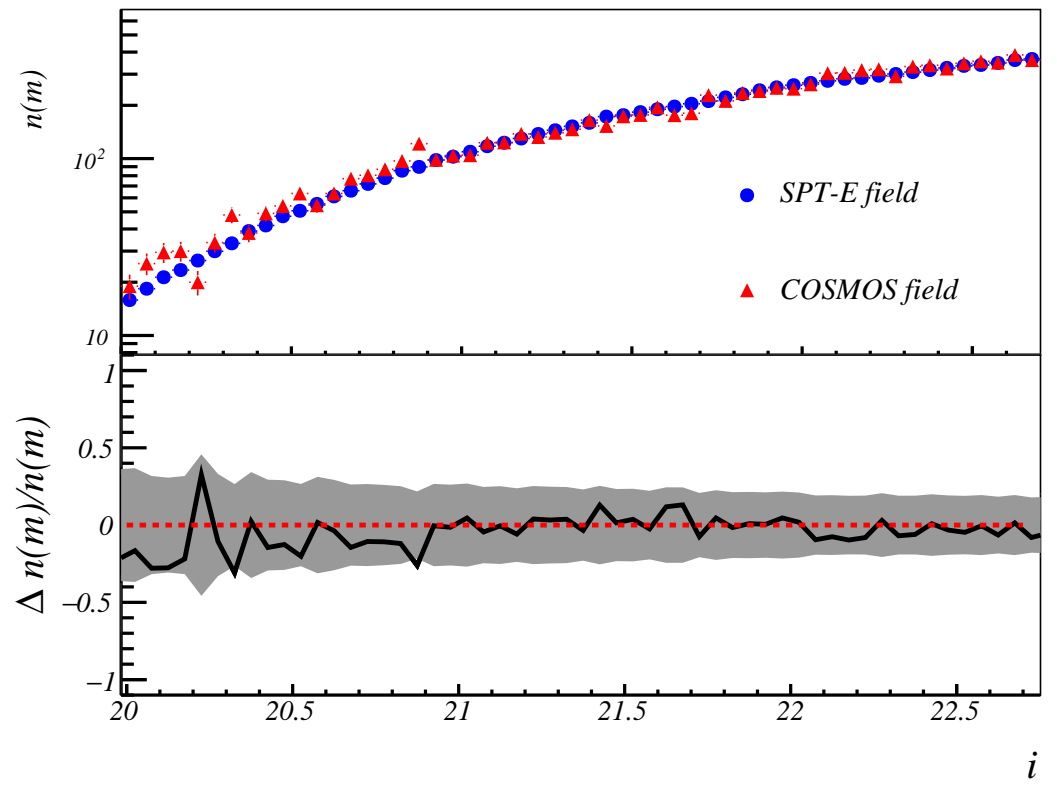

Figure B.2: Upper panel: Comparison of the magnitude distribution for the SPT-E and the COSMOS fields for the $z$ band. 


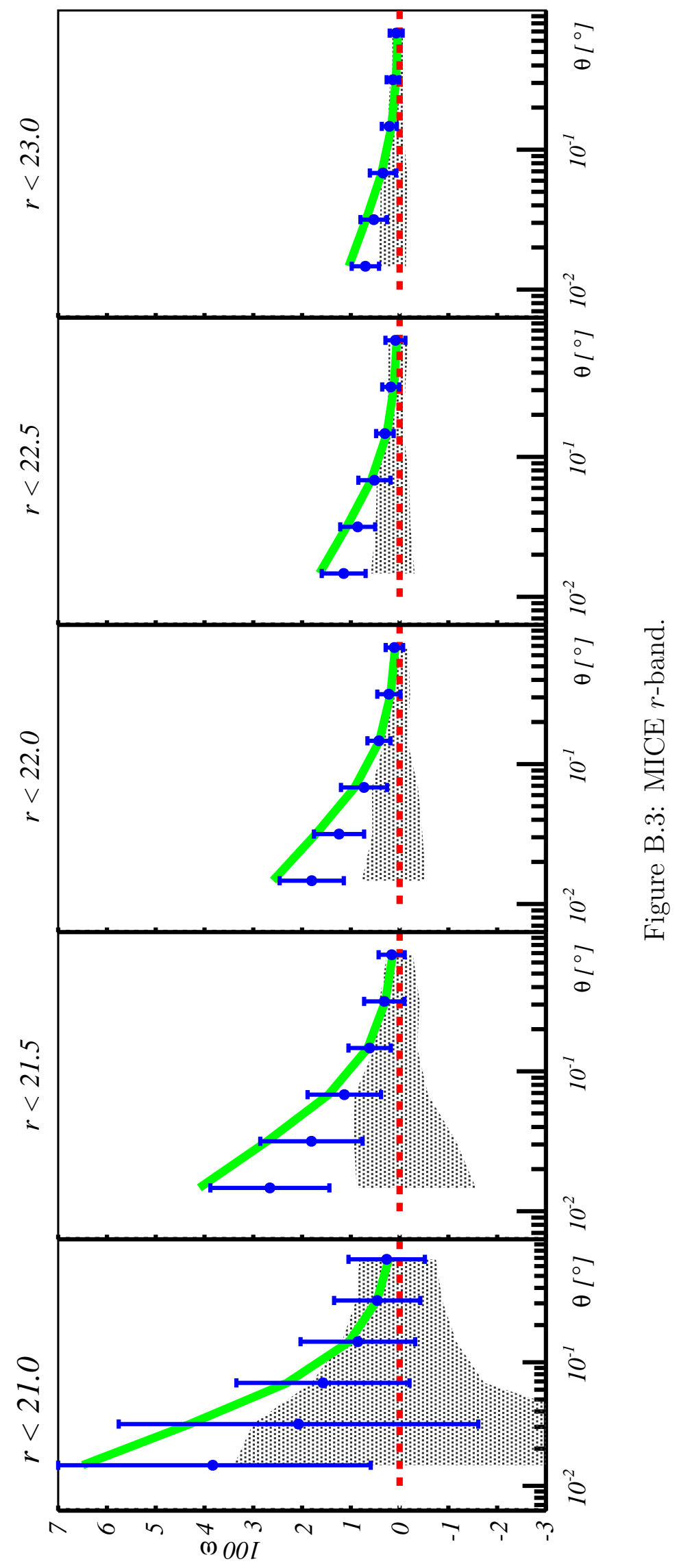




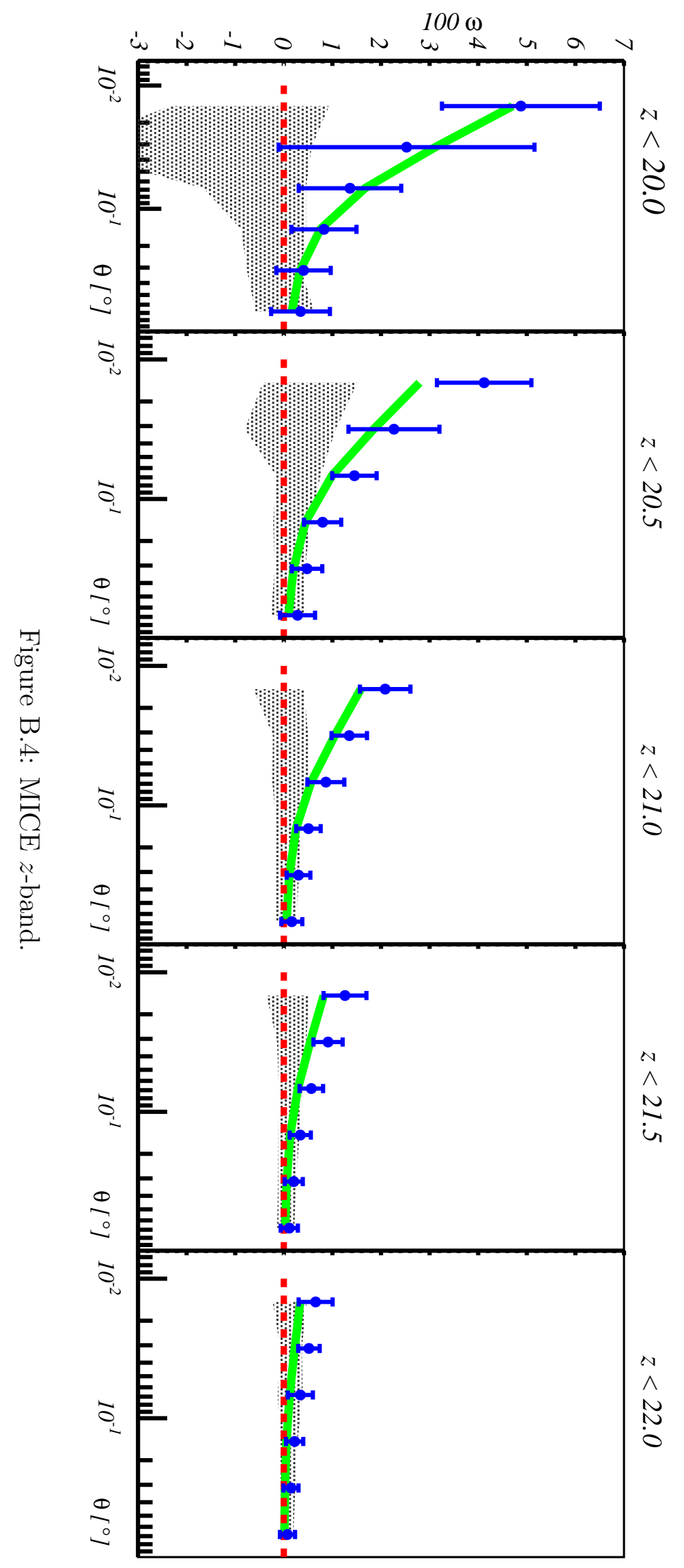




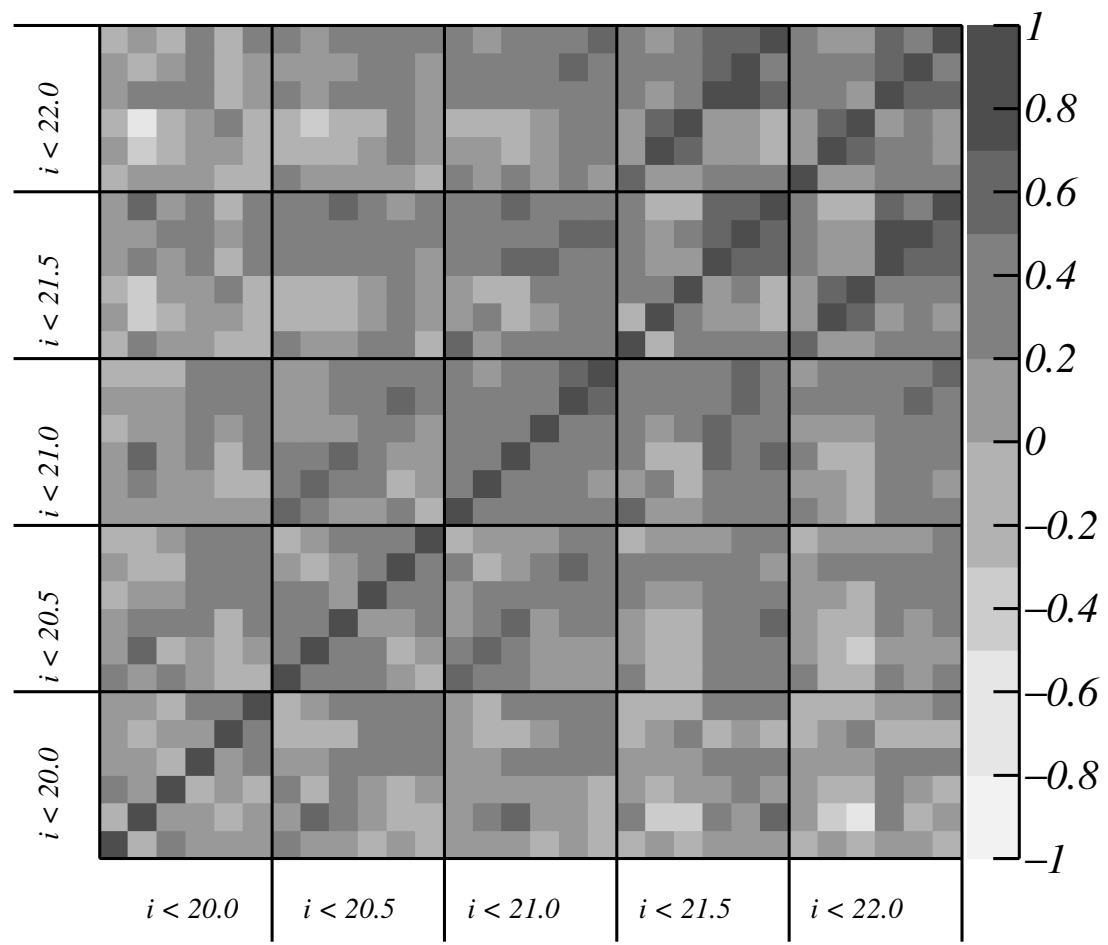

Figure B.5: Covariance matrix of the $r$-band rescaled by the value of the diagonal.

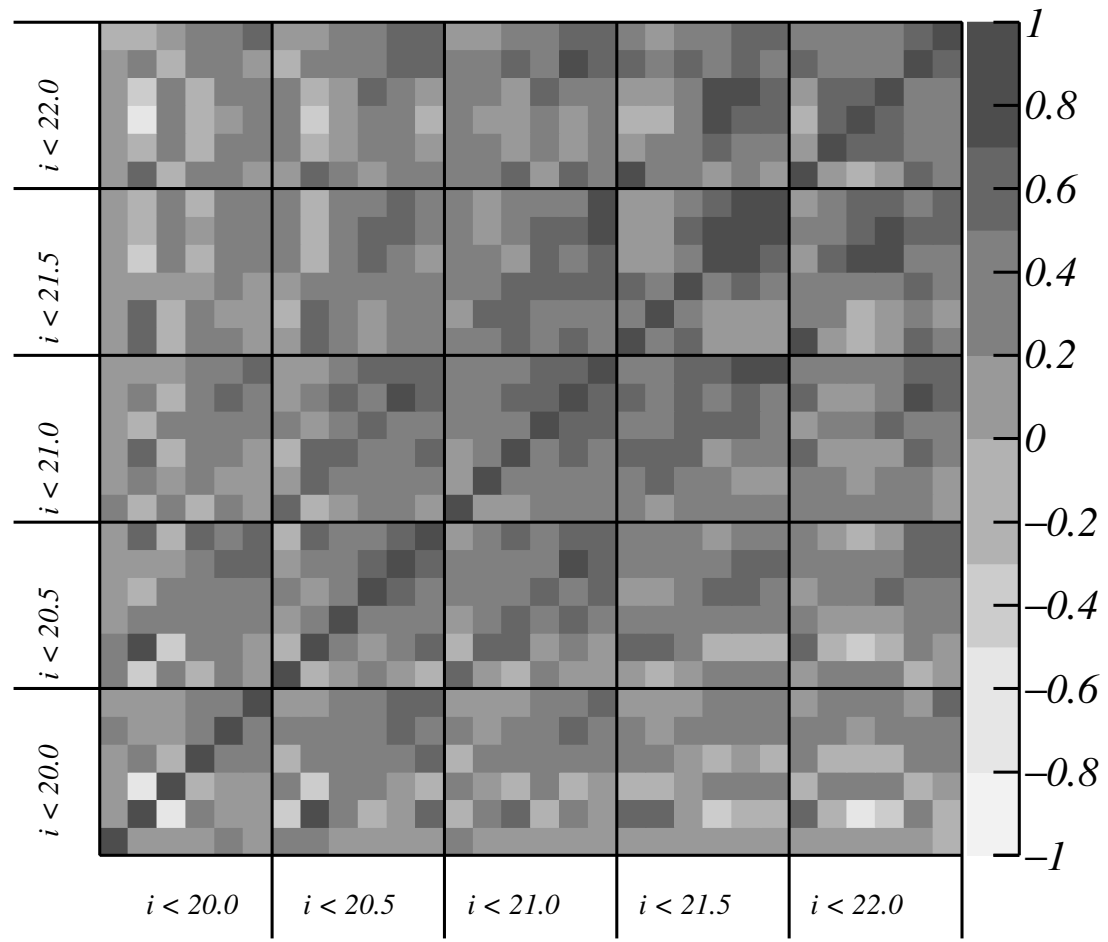

Figure B.6: Covariance matrix of the $z$-band rescaled by the value of the diagonal. 


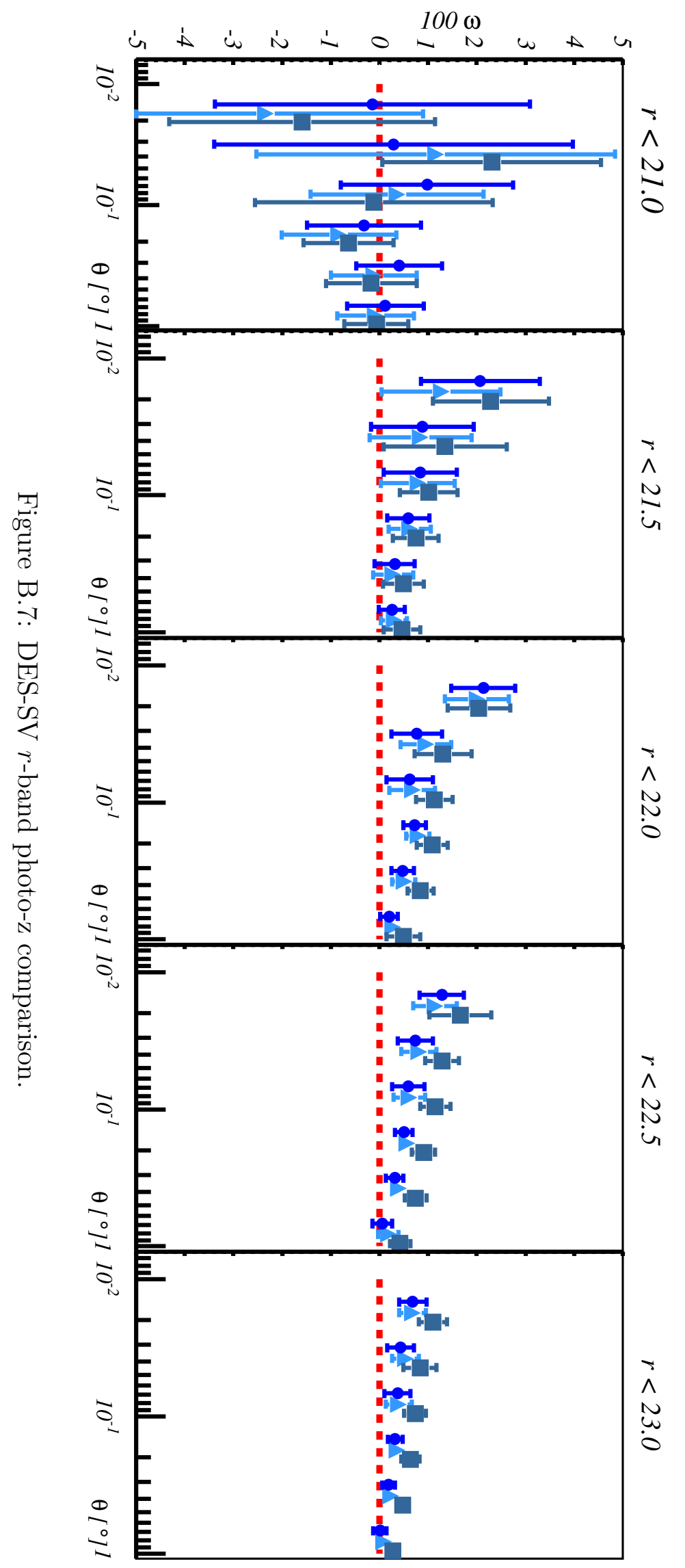




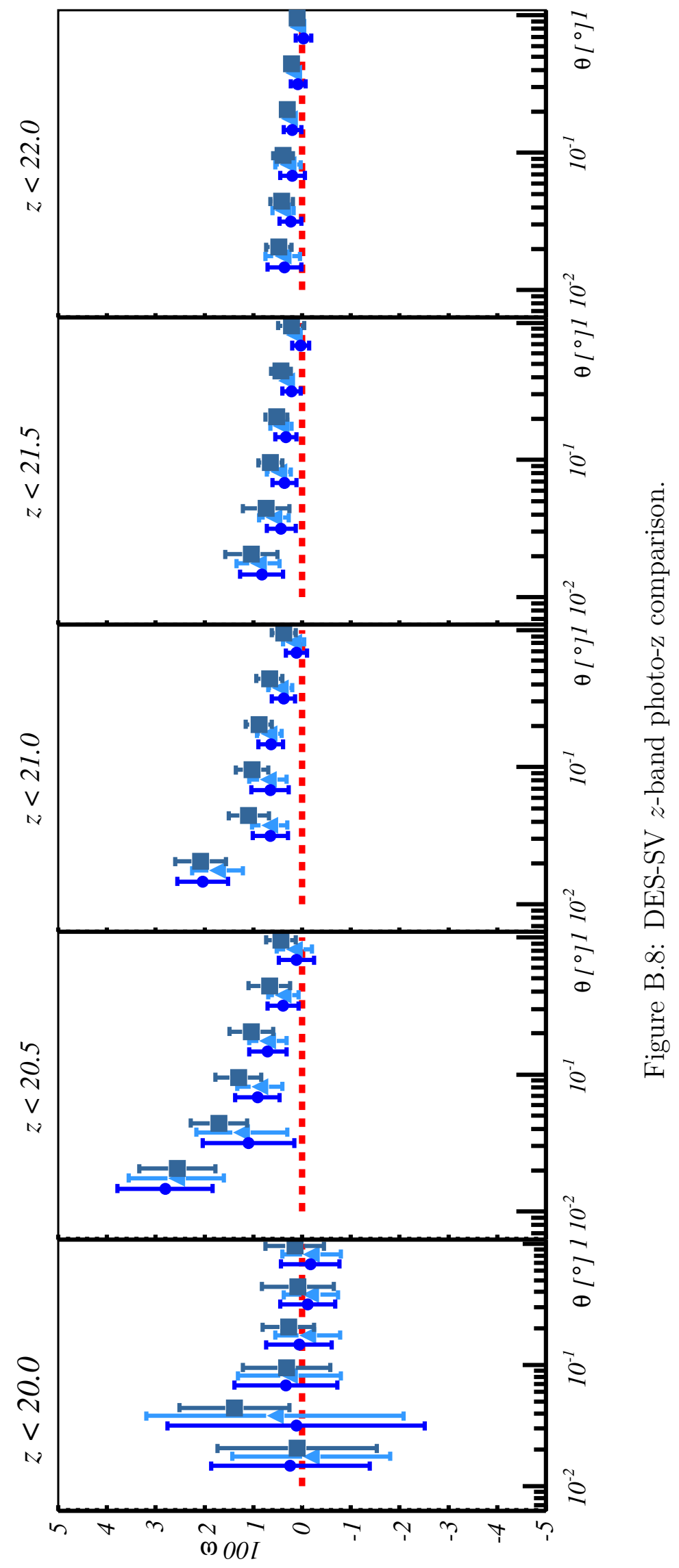




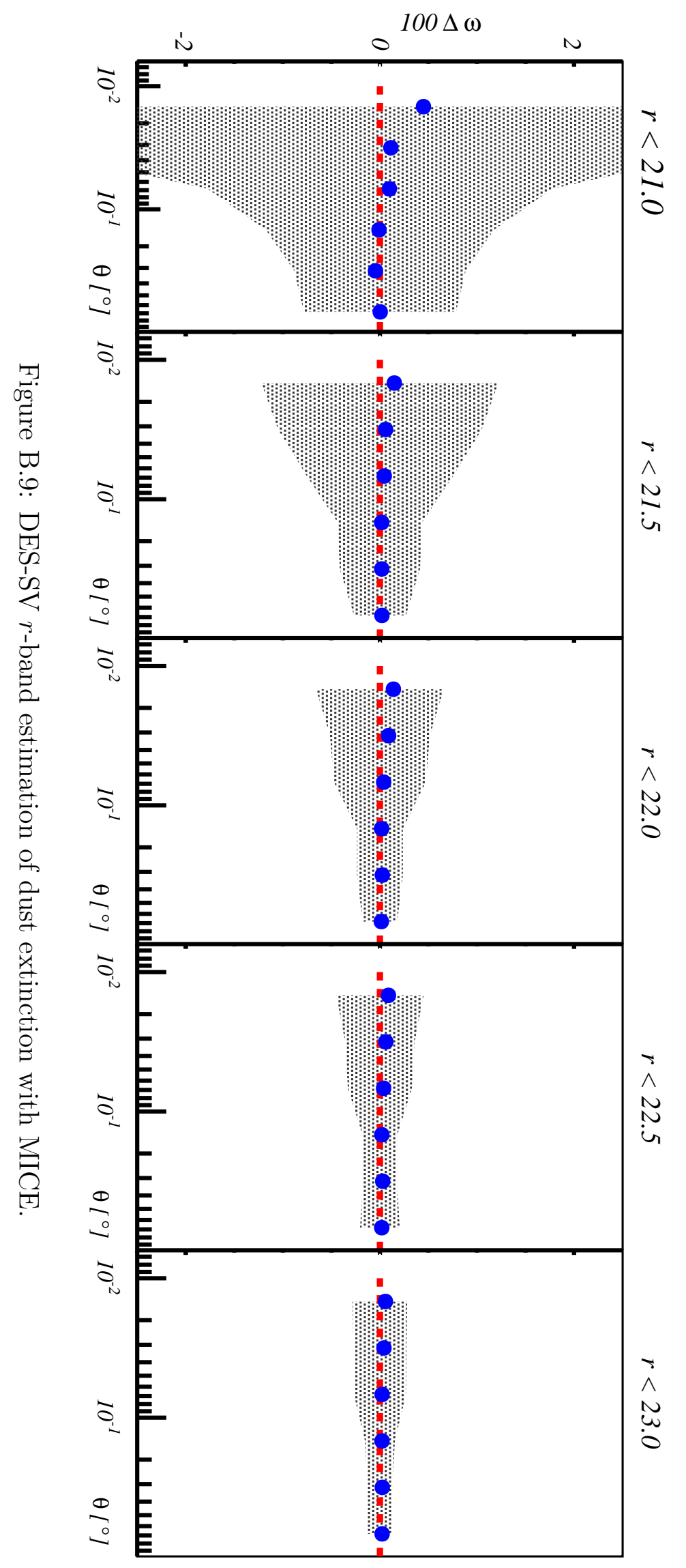




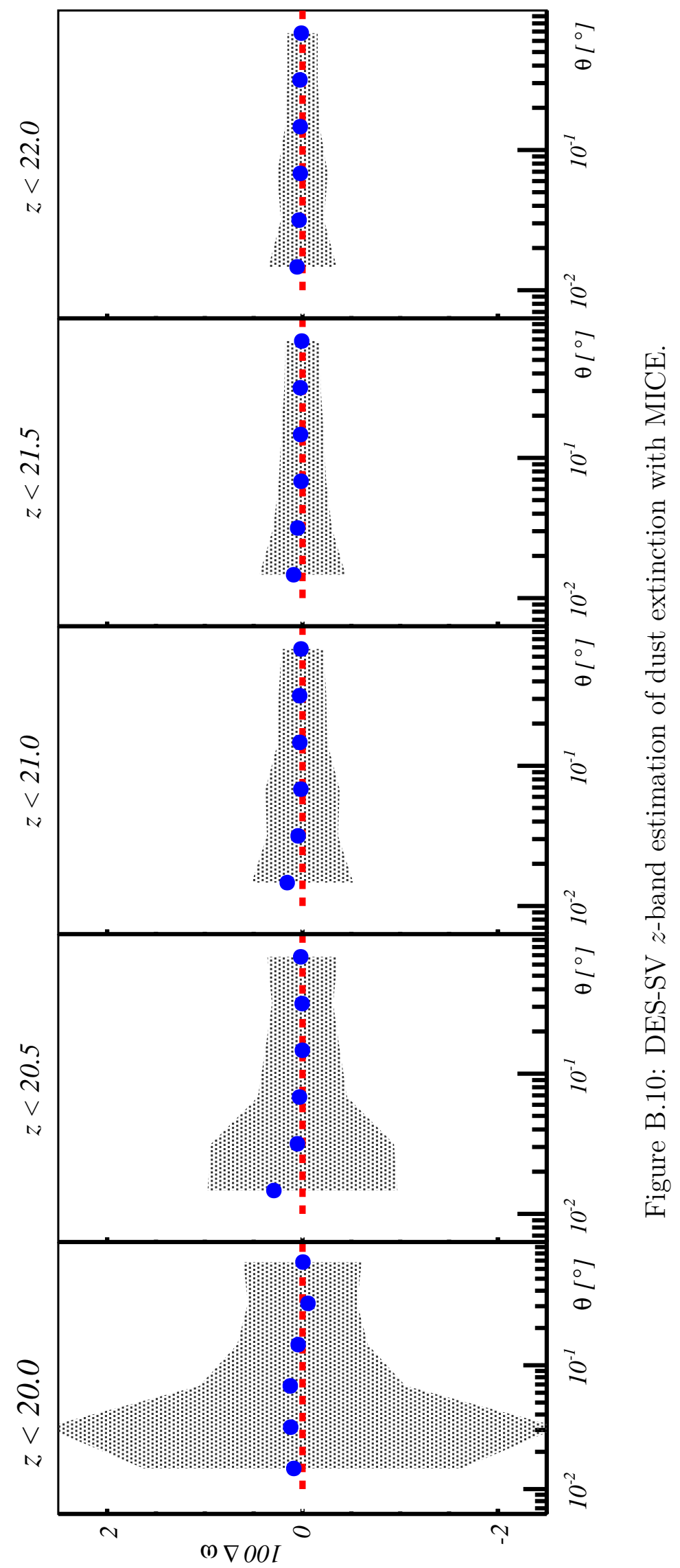




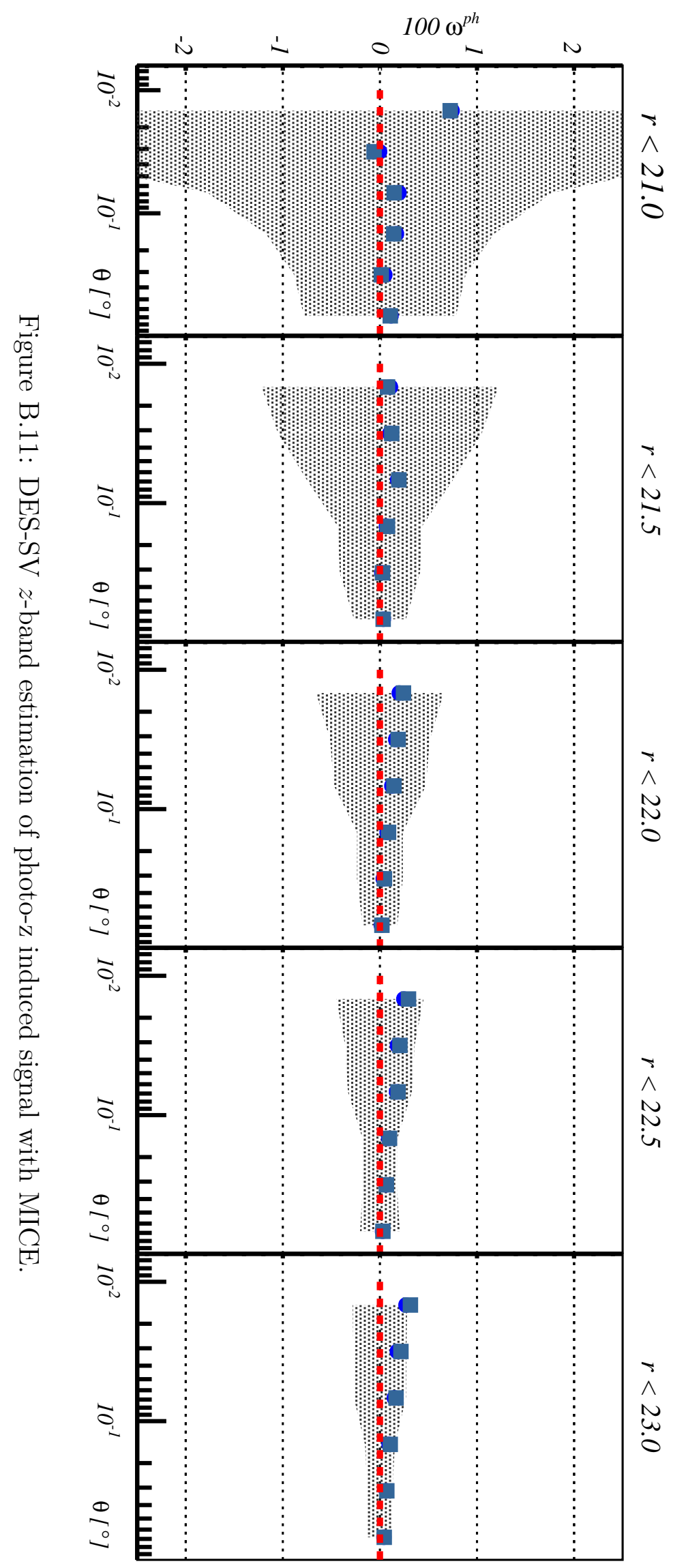




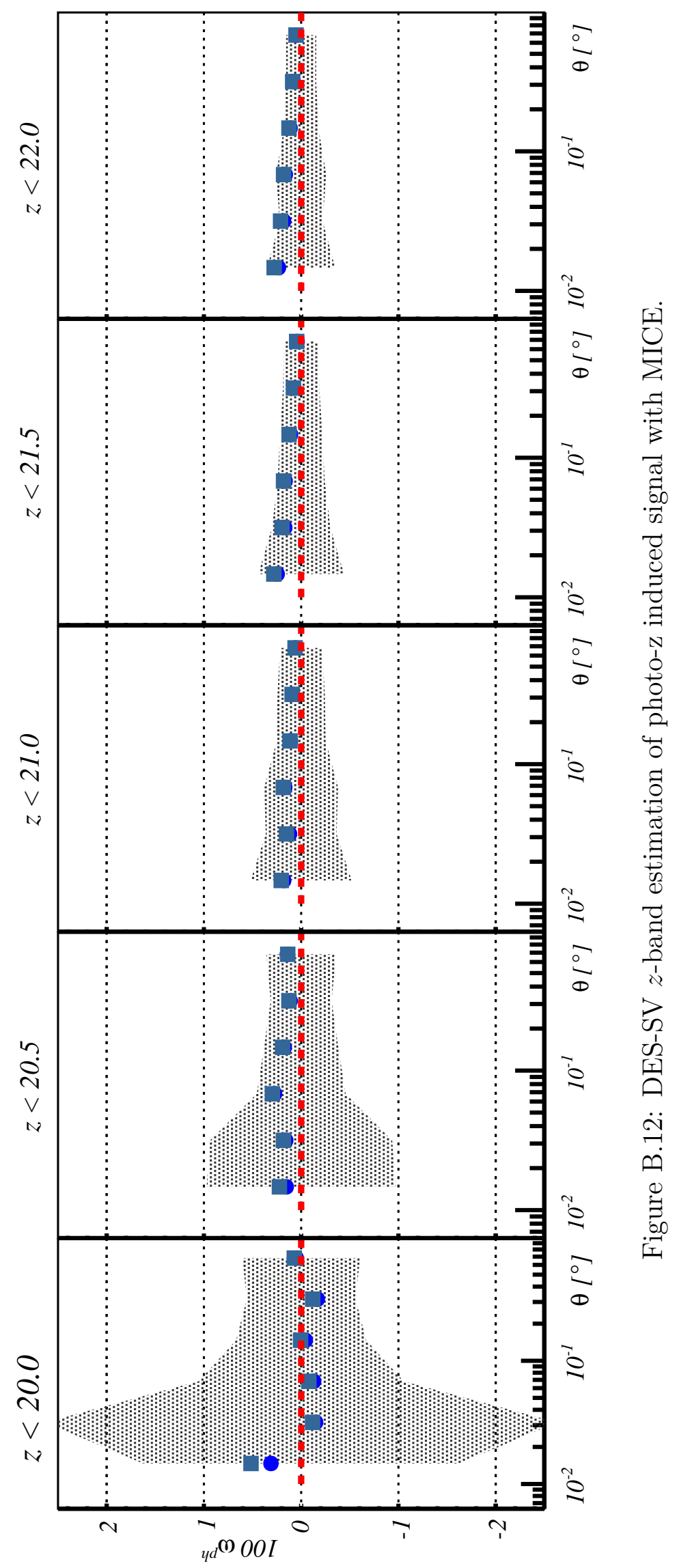




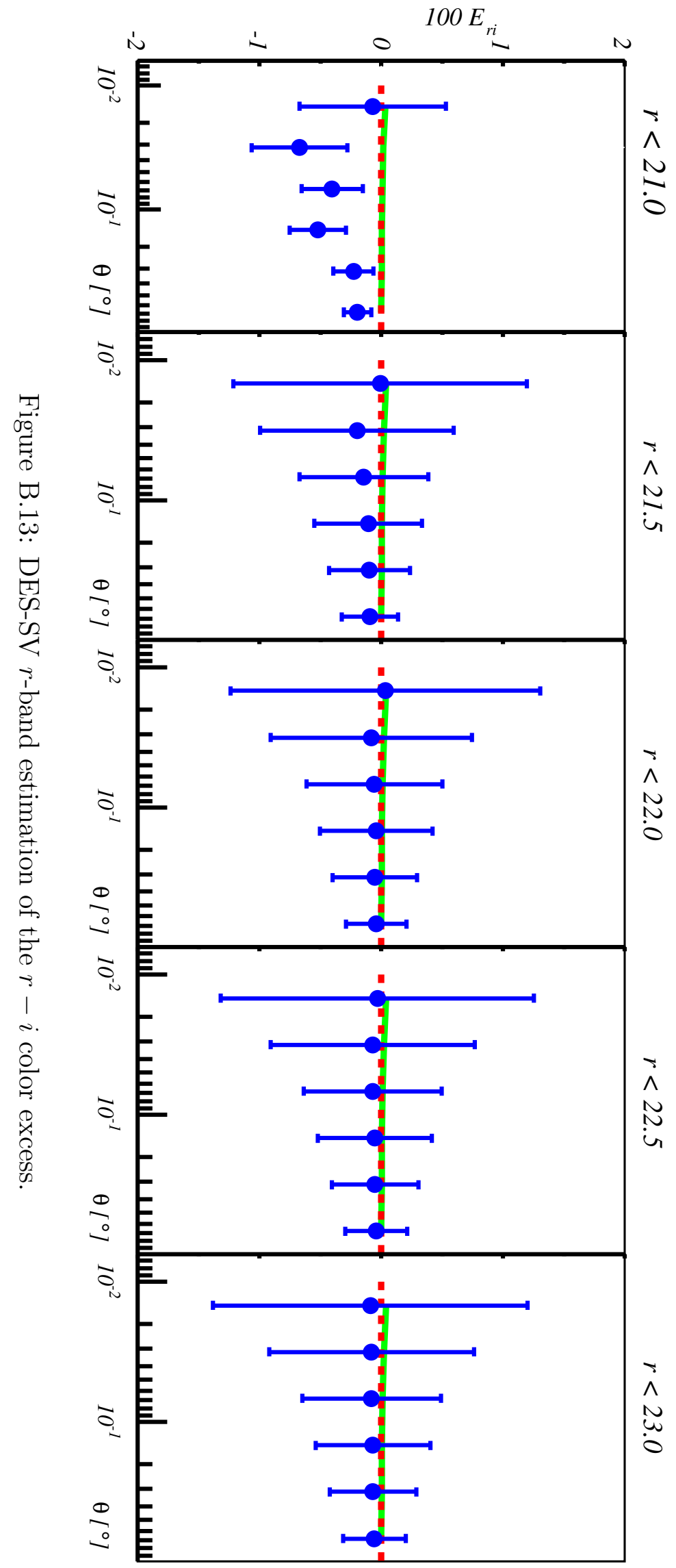




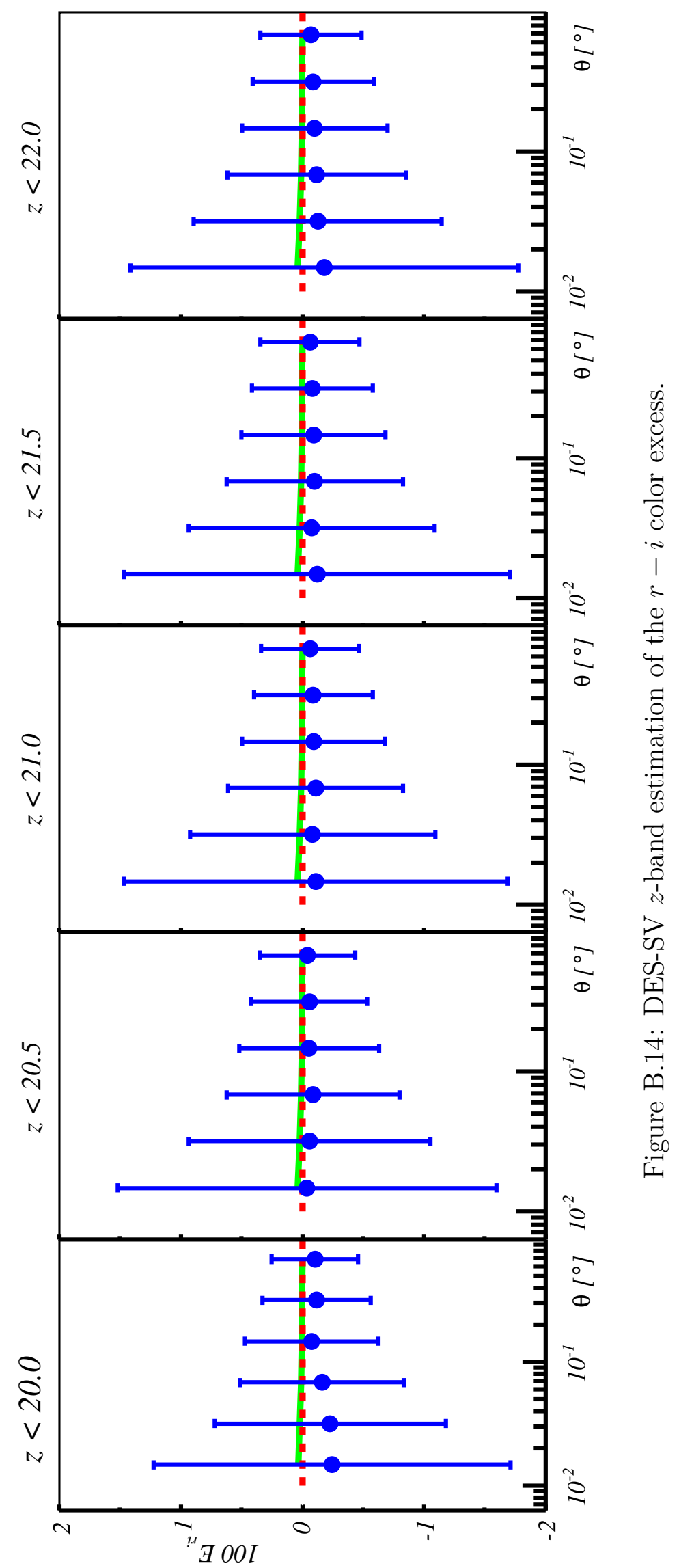




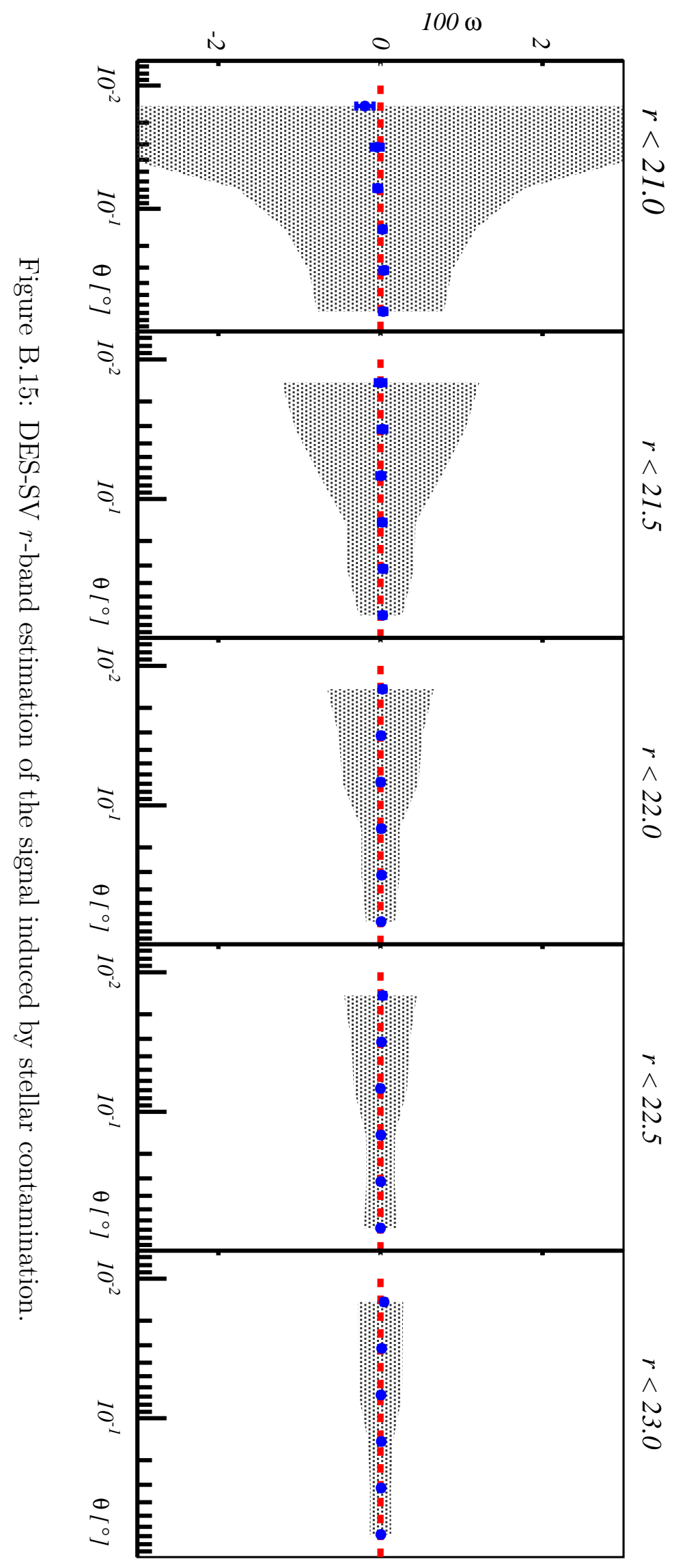




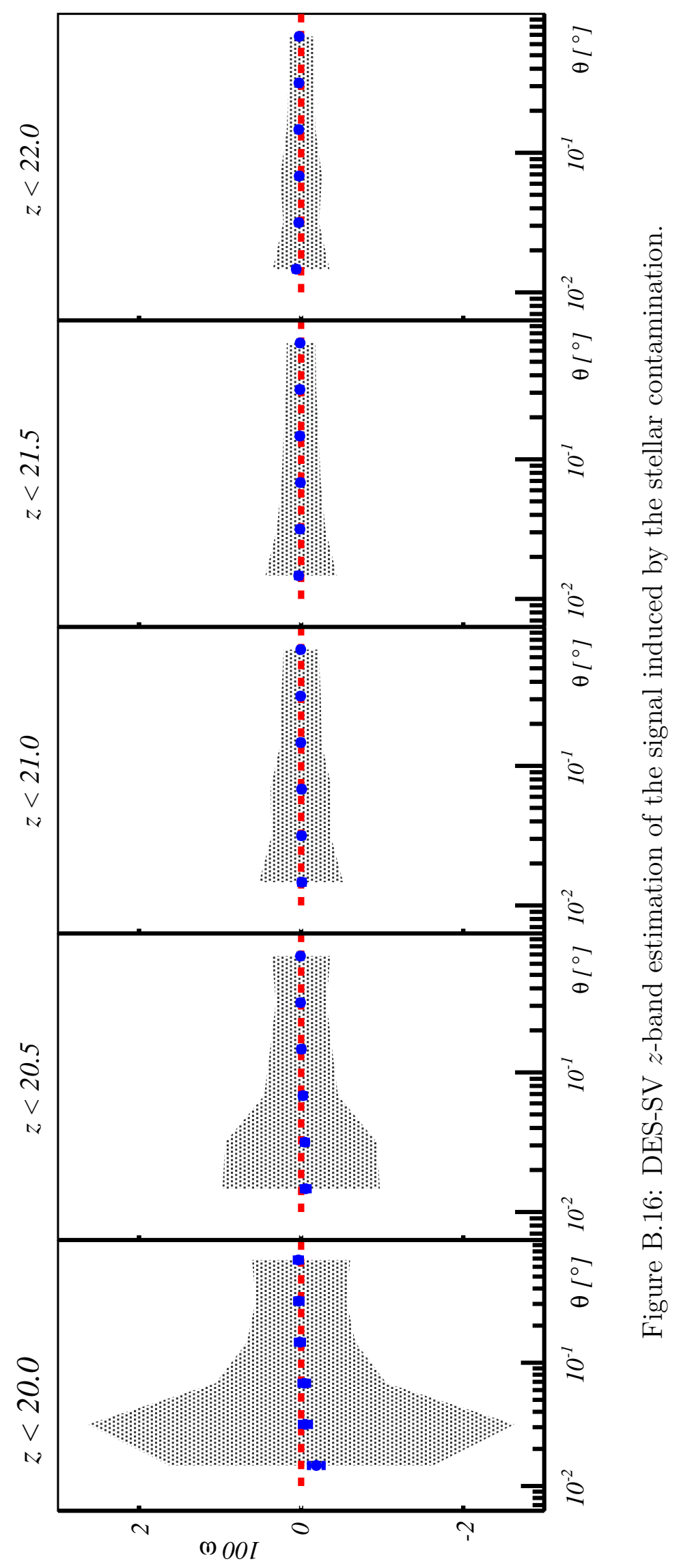





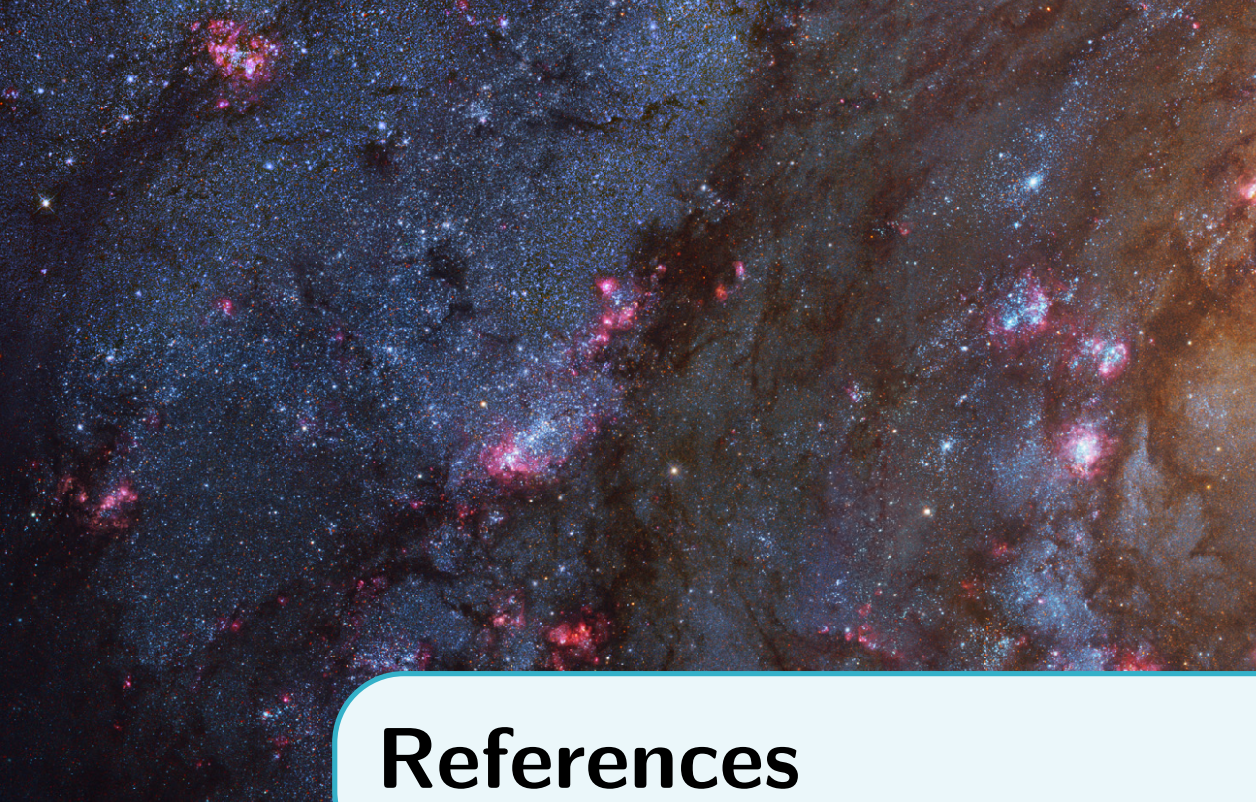

4.8:

[1] F. Halzen and A.D. Martin. Quarks and leptons: an introductory course in modern particle physics. Wiley, 1984. URL: https://books.google.es/ books? id=zwDvAAAAMAAJ.

[2] M.E. Peskin and D.V. Schroeder. An Introduction to Quantum Field Theory. Advanced book classics. Avalon Publishing, 1995. ISBN: 9780201503975. URL: https://books.google.es/books?id=i35LALNOGosC.

[3] S. Weinberg. The Quantum Theory of Fields. The Quantum Theory of Fields 3 Volume Hardback Set v. 1. Cambridge University Press, 1995. ISBN: 9780521550017. URL: https://books.google.es/books?id=doeDB3\%5C_ WLvwC.

[4] S. Weinberg. The Quantum Theory of Fields: Volume 2, Modern Applications. Cambridge University Press, 1996. ISBN: 9781139643252. URL: https://books.google.es/books?id=J2EhAwAAQBAJ.

[5] S. F. Novaes. "Standard Model: An Introduction". In: ArXiv High Energy Physics - Phenomenology e-prints (Jan. 2000). eprint: hep-ph/0001283.

[6] A. Einstein. "Die Grundlage der allgemeinen Relativitätstheorie". In: $A n$ nalen der Physik 354.7 (1916), pp. 769-822. ISSN: 1521-3889. DOI: 10 . 1002 / andp . 19163540702. URL: http : / / dx . doi . org/10 . 1002 / andp . 19163540702.

[7] A. Einstein. "Die Grundlage der allgemeinen Relativitätstheorie". In: $A n$ nalen der Physik 354 (1916), pp. 769-822. DOI: 10.1002/andp. 19163540702.

[8] A. Einstein. "Kosmologische Betrachtungen zur allgemeinen Relativitätstheorie". In: Sitzungsberichte der Königlich Preußischen Akademie der Wissenschaften (Berlin), Seite 142-152. (1917).

[9] S. M. Carroll. "The Cosmological Constant". In: Living Reviews in Relativity 4, 1 (Dec. 2001), p. 1. DOI: 10.12942/lrr-2001-1. eprint: astro-ph/ 0004075 . 
[10] David Hilbert. "Die Grundlagen der Physik. 1." In: Gott. Nachr. 27 (1915), pp. 395-407.

[11] D. Alonso et al. "Measuring the transition to homogeneity with photometric redshift surveys". In: MNRAS 440 (May 2014), pp. 10-23. DOI: 10.1093/ mnras/stu255. arXiv: 1312.0861 [astro-ph.CO].

[12] D. Alonso et al. "Homogeneity and isotropy in the Two Micron All Sky Survey Photometric Redshift catalogue". In: MNRAS 449 (May 2015), pp. 670-684. DOI: 10.1093/mnras/stv309. arXiv: 1412.5151.

[13] G. Lemaître. "Un Univers homogène de masse constante et de rayon croissant rendant compte de la vitesse radiale des nébuleuses extra-galactiques". In: Annales de la Société Scientifique de Bruxelles 47 (1927), pp. 49-59.

[14] M. Betoule et al. "Improved cosmological constraints from a joint analysis of the SDSS-II and SNLS supernova samples". In: A\&A 568, A22 (Aug. 2014), A22. DOI: 10.1051/0004-6361/201423413. arXiv: 1401.4064.

[15] D. Burstein et al. "The distribution of mass in SC galaxies". In: ApJ 253 (Feb. 1982), pp. 70-85. DOI: 10.1086/159611.

[16] V. C. Rubin. "The rotation of spiral galaxies". In: Science 220 (June 1983), pp. 1339-1344. DOI: 10.1126/science.220.4604.1339.

[17] D. Burstein and V. C. Rubin. "The distribution of mass in spiral galaxies". In: ApJ 297 (Oct. 1985), pp. 423-435. DOI: 10.1086/163541.

[18] V. C. Rubin et al. "Rotation velocities of 16 SA galaxies and a comparison of Sa, Sb, and SC rotation properties". In: ApJ 289 (Feb. 1985), pp. 81-98. DOI: $10.1086 / 162866$.

[19] T. Marrodán Undagoitia and L. Rauch. "Dark matter direct-detection experiments". In: Journal of Physics G Nuclear Physics 43.1, 013001 (Jan. 2016), p. 013001. DOI: 10.1088/0954-3899/43/1/013001. arXiv: 1509 . 08767 [physics.ins-det].

[20] J. M. Gaskins. "A review of indirect searches for particle dark matter". In: Contemporary Physics 57 (Oct. 2016), pp. 496-525. DOI: 10.1080/ 00107514.2016.1175160. arXiv: 1604.00014 [astro-ph.HE].

[21] E. Hubble. "A Relation between Distance and Radial Velocity among ExtraGalactic Nebulae". In: Proceedings of the National Academy of Science 15 (Mar. 1929), pp. 168-173. DOI: 10.1073/pnas.15.3.168.

[22] S. Perlmutter et al. "Measurements of $\Omega$ and $\Lambda$ from 42 High-Redshift Supernovae". In: ApJ 517 (June 1999), pp. 565-586. DOI: 10.1086/307221. eprint: astro-ph/9812133.

[23] G. Steigman. "Primordial Nucleosynthesis:. Successes and Challenges". In: International Journal of Modern Physics E 15 (2006), pp. 1-35. DOI: 10.1142/S0218301306004028. eprint: astro-ph/0511534.

[24] Planck Collaboration et al. "Planck 2015 results. XIII. Cosmological parameters". In: ArXiv e-prints (Feb. 2015). arXiv: 1502.01589. 
[25] Ya B Zel'dovich. "THE COSMOLOGICAL CONSTANT AND THE THEORY OF ELEMENTARY PARTICLES". In: Soviet Physics Uspekhi 11.3 (1968), p. 381. URL: http://stacks.iop.org/0038-5670/11/i=3/a=A13.

[26] Steven Weinberg. "The cosmological constant problem". In: Rev. Mod. Phys. 61 (1 Jan. 1989), pp. 1-23. DOI: 10.1103/RevModPhys.61.1. URL: https://link.aps.org/doi/10.1103/RevModPhys.61.1.

[27] T. Padmanabhan. "Cosmological constant-the weight of the vacuum". In: Phys. Rep. 380 (July 2003), pp. 235-320. DOI: 10.1016/S0370-1573(03) 00120-0. eprint: hep-th/0212290.

[28] R. L. Jaffe. "Casimir effect and the quantum vacuum". In: Phys. Rev. D 72 (2 July 2005), p. 021301. DOI: 10.1103/PhysRevD . 72.021301. URL: https://link.aps.org/doi/10.1103/PhysRevD.72.021301.

[29] A. D. Linde. "Is the Lee constant a cosmological constant?" In: Soviet Journal of Experimental and Theoretical Physics Letters 19 (Mar. 1974), p. 183.

[30] M. Veltman. "Cosmology and the Higgs Mass". In: Phys. Rev. Lett. 34 (12 Mar. 1975), pp. 777-777. DOI: 10.1103/PhysRevLett. 34.777. URL: https://link.aps.org/doi/10.1103/PhysRevLett.34.777.

[31] L. Amendola and S. Tsujikawa. Dark Energy: Theory and Observations. 2010.

[32] A. De Felice and S. Tsujikawa. " $\mathrm{f}(\mathrm{R})$ Theories". In: Living Reviews in Relativity 13, 3 (Dec. 2010), p. 3. DOI: 10.12942/lrr-2010-3. arXiv: 1002.4928 [gr-qc].

[33] T. P. Sotiriou and V. Faraoni. " $\mathrm{f}(\mathrm{R})$ theories of gravity". In: Reviews of Modern Physics 82 (Jan. 2010), pp. 451-497. DOI: 10.1103/RevModPhys. 82.451. arXiv: 0805.1726 [gr-qc].

[34] Mohamed Abdelwahab, Rituparno Goswami, and Peter K. S. Dunsby. "Cosmological dynamics of fourth-order gravity: A compact view". In: Phys. Rev. D 85 (8 Apr. 2012), p. 083511. DOI: 10.1103/PhysRevD.85.083511. URL: https://link.aps.org/doi/10.1103/PhysRevD.85.083511.

[35] A. M. Nzioki et al. "Geometrical approach to strong gravitational lensing in $\mathrm{f}(\mathrm{R})$ gravity". In: Phys. Rev. D 83.2, 024030 (Jan. 2011), p. 024030. DOI: 10.1103/PhysRevD.83.024030. arXiv: 1002.2056 [gr-qc].

[36] R. Gavazzi et al. "The Sloan Lens ACS Survey. VI. Discovery and Analysis of a Double Einstein Ring". In: ApJ 677, 1046-1059 (Apr. 2008), pp. 10461059. DOI: $10.1086 / 529541$. arXiv: 0801.1555.

[37] E. Berti et al. "Testing general relativity with present and future astrophysical observations". In: Classical and Quantum Gravity 32.24, 243001 (Dec. 2015), p. 243001. DOI: 10.1088/0264-9381/32/24/243001. arXiv: 1501.07274 [gr-qc].

[38] C. D. Leonard, T. Baker, and P. G. Ferreira. "Exploring degeneracies in modified gravity with weak lensing". In: Phys. Rev. D 91.8, 083504 (Apr. 2015), p. 083504. DOI: 10.1103/PhysRevD.91.083504. arXiv: 1501.03509. 
[39] David H. Weinberg et al. "Observational probes of cosmic acceleration". In: Physics Reports 530.2 (2013). Observational Probes of Cosmic Acceleration, pp. 87-255. ISSN: 0370-1573. DOI: http://dx . doi .org/10 . 1016/ j . physrep.2013.05.001. URL: http://www.sciencedirect.com/science/ article/pii/S0370157313001592.

[40] C. Alcock and B. Paczynski. "An evolution free test for non-zero cosmological constant". In: Nature 281 (Oct. 1979), p. 358. DOI: 10.1038/281358a0.

[41] C. Yèche et al. "Prospects for dark energy evolution: a frequentist multiprobe approach". In: $A \& A A 48$ (Mar. 2006), pp. 831-842. DOI: 10.1051/ 0004-6361:20053786. eprint: astro-ph/0507170.

[42] Planck Collaboration et al. "Planck 2015 results. XIV. Dark energy and modified gravity". In: $A \mathscr{E} A$ 594, A14 (Sept. 2016), A14. DOI: 10.1051/00046361/201525814. arXiv: 1502.01590.

[43] Metin Ata et al. "The clustering of the SDSS-IV extended Baryon Oscillation Spectroscopic Survey DR14 quasar sample: First measurement of Baryon Acoustic Oscillations between redshift 0.8 and 2.2". In: (2017). arXiv: 1705.06373 [astro-ph.CO].

[44] E. A. Kazin et al. "The WiggleZ Dark Energy Survey: improved distance measurements to $\mathrm{z}=1$ with reconstruction of the baryonic acoustic feature". In: MNRAS 441 (July 2014), pp. 3524-3542. DOI: 10.1093/mnras/stu778. arXiv: 1401.0358.

[45] A. J. Ross et al. "The clustering of the SDSS DR7 main Galaxy sample - I. A 4 per cent distance measure at $\mathrm{z}=0.15$ ". In: MNRAS 449 (May 2015), pp. 835-847. DOI: 10.1093/mnras/stv154. arXiv: 1409.3242.

[46] F. Beutler et al. "The 6dF Galaxy Survey: baryon acoustic oscillations and the local Hubble constant". In: MNRAS 416 (Oct. 2011), pp. 3017-3032. DOI: $10.1111 / \mathrm{j} .1365-2966.2011 .19250 . \mathrm{x}$. arXiv: 1106.3366.

[47] X.-D. Li et al. "Cosmological Constraints from the Redshift Dependence of the Alcock-Paczynski Effect: Application to the SDSS-III BOSS DR12 Galaxies". In: ApJ 832, 103 (Dec. 2016), p. 103. DOI: 10.3847 / $0004-$ 637X/832/2/103. arXiv: 1609.05476.

[48] S. Alam et al. "The clustering of galaxies in the completed SDSS-III Baryon Oscillation Spectroscopic Survey: cosmological analysis of the DR12 galaxy sample". In: ArXiv e-prints (July 2016). arXiv: 1607.03155.

[49] Y. Wang et al. "The clustering of galaxies in the completed SDSS-III Baryon Oscillation Spectroscopic Survey: tomographic BAO analysis of DR12 combined sample in configuration space". In: ArXiv e-prints (July 2016). arXiv: 1607.03154.

[50] J. E. Bautista et al. "Measurement of BAO correlations at $z=2.3$ with SDSS DR12 \lya-Forests". In: ArXiv e-prints (Feb. 2017). arXiv: 1702.00176. 
[51] H. Gil-Marín et al. "The clustering of galaxies in the SDSS-III Baryon Oscillation Spectroscopic Survey: RSD measurement from the power spectrum and bispectrum of the DR12 BOSS galaxies". In: MNRAS 465 (Feb. 2017), pp. 1757-1788. DOI: $10.1093 / \mathrm{mnras} / \mathrm{stw} 2679$. arXiv: 1606.00439.

[52] A. G. Riess et al. "A 2.4\% Determination of the Local Value of the Hubble Constant". In: ApJ 826, 56 (July 2016), p. 56. DOI: 10.3847 / $0004-$ 637X/826/1/56. arXiv: 1604.01424.

[53] M. Kilbinger et al. "CFHTLenS: combined probe cosmological model comparison using 2D weak gravitational lensing". In: MNRAS 430 (Apr. 2013), pp. 2200-2220. DOI: 10.1093/mnras/stt041. arXiv: 1212.3338.

[54] J. Harnois-Déraps et al. "KiDS-450: Tomographic Cross-Correlation of Galaxy Shear with $\{\backslash$ it Planck\} Lensing". In: ArXiv e-prints (Mar. 2017). arXiv: 1703.03383.

[55] A. Leauthaud et al. "Lensing is low: cosmology, galaxy formation or new physics?" In: MNRAS 467 (May 2017), pp. 3024-3047. DOI: 10.1093/ mnras/stx258. arXiv: 1611.08606.

[56] S. Joudaki et al. "KiDS-450: Testing extensions to the standard cosmological model". In: ArXiv e-prints (Oct. 2016). arXiv: 1610.04606.

[57] E. Di Valentino et al. "Constraining Dark Energy Dynamics in Extended Parameter Space". In: ArXiv e-prints (Apr. 2017). arXiv: 1704.00762.

[58] G.-B. Zhao et al. "The clustering of galaxies in the completed SDSS-III Baryon Oscillation Spectroscopic Survey: Examining the observational evidence for dynamical dark energy". In: ArXiv e-prints (Jan. 2017). arXiv: 1701.08165.

[59] J. N. Dossett et al. "Constraints and tensions in testing general relativity from Planck and CFHTLenS data including intrinsic alignment systematics". In: Phys. Rev. D 92.2, 023003 (July 2015), p. 023003. DOI: 10.1103 / PhysRevD.92.023003. arXiv: 1501.03119.

[60] S. Joudaki et al. "CFHTLenS revisited: assessing concordance with Planck including astrophysical systematics". In: MNRAS 465 (Feb. 2017), pp. 20332052. DOI: 10.1093/mnras/stw2665. arXiv: 1601.05786.

[61] B. Li, G.-B. Zhao, and K. Koyama. "Haloes and voids in $f(R)$ gravity". In: MNRAS 421 (Apr. 2012), pp. 3481-3487. DOI: $10.1111 / \mathrm{j} .1365-$ 2966.2012.20573.x. arXiv: 1111.2602 [astro-ph.CO].

[62] F. von Braun-Bates et al. "The $\mathrm{f}$ (Script $\mathrm{R}$ ) halo mass function in the cosmic web". In: J. Cosmology Astropart. Phys. 3, 012 (Mar. 2017), p. 012. DoI: 10.1088/1475-7516/2017/03/012. arXiv: 1702.06817.

[63] G. Lavaux and B. D. Wandelt. "Precision cosmology with voids: definition, methods, dynamics". In: MNRAS 403 (Apr. 2010), pp. 1392-1408. DOI: 10.1111/j.1365-2966.2010.16197.x. arXiv: 0906.4101.

[64] Guilhem Lavaux and Benjamin D. Wandelt. "Precision Cosmography with Stacked Voids". In: The Astrophysical Journal 754.2 (2012), p. 109. URL: http://stacks.iop.org/0004-637X/754/i=2/a=109. 
[65] Daeseong Park and Jounghun Lee. "Void Ellipticity Distribution as a Probe of Cosmology". In: Phys. Rev. Lett. 98 (8 Feb. 2007), p. 081301. DoI: 10.1103/PhysRevLett.98.081301. URL: https://link.aps .org/doi/ 10.1103/PhysRevLett.98.081301.

[66] D. Spolyar, M. Sahlén, and J. Silk. "Topology and Dark Energy: Testing Gravity in Voids". In: Physical Review Letters 111.24, 241103 (Dec. 2013), p. 241103. DOI: 10.1103/PhysRevLett.111.241103. arXiv: 1304.5239 [astro-ph.CO].

[67] E. V. Arbuzova, A. D. Dolgov, and L. Reverberi. "Spherically symmetric solutions in F (R) gravity and gravitational repulsion". In: Astroparticle Physics 54 (Feb. 2014), pp. 44-47. DOI: 10.1016/j.astropartphys. 2013. 11.008. arXiv: 1306.5694 [gr-qc].

[68] Y.-C. Cai, N. Padilla, and B. Li. "Testing Gravity using Void Profiles". In: ArXiv e-prints (Oct. 2014). arXiv: 1410.8355.

[69] P. Zivick et al. "Using cosmic voids to distinguish $\mathrm{f}(\mathrm{R})$ gravity in future galaxy surveys". In: MNRAS 451 (Aug. 2015), pp. 4215-4222. DOI: 10 . 1093/mnras/stv1209. arXiv: 1411.5694.

[70] A. Barreira et al. "Weak lensing by voids in modified lensing potentials". In: J. Cosmology Astropart. Phys. 8, 028 (Aug. 2015), p. 028. DOI: 10.1088/ 1475-7516/2015/08/028. arXiv: 1505.05809.

[71] I. Achitouv et al. "Imprint of $\mathrm{f}(\mathrm{R})$ gravity on nonlinear structure formation". In: Phys. Rev. D 93.10, 103522 (May 2016), p. 103522. DOI: 10.1103/ PhysRevD.93.103522. arXiv: 1511.01494.

[72] I. Achitouv. "Testing the imprint of nonstandard cosmologies on void profiles using Monte Carlo random walks". In: Phys. Rev. D 94.10, 103524 (Nov. 2016), p. 103524. DOI: 10.1103/PhysRevD.94.103524. arXiv: 1609.01284.

[73] F. W. Dyson, A. S. Eddington, and C. Davidson. "A Determination of the Deflection of Light by the Sun's Gravitational Field, from Observations Made at the Total Eclipse of May 29, 1919". In: Philosophical Transactions of the Royal Society of London A: Mathematical, Physical and Engineering Sciences 220.571-581 (1920), pp. 291-333. ISSN: 0264-3952. DOI: 10 . 1098/rsta.1920.0009. eprint: http://rsta. royalsocietypublishing . org / content / 220 / 571-581/291. full . pdf. URL: http : / / rsta . royalsocietypublishing. org/content/220/571-581/291.

[74] B. Nord et al. "Observation and Confirmation of Six Strong-lensing Systems in the Dark Energy Survey Science Verification Data". In: ApJ 827, 51 (Aug. 2016), p. 51. DOI: $10.3847 / 0004-637 X / 827 / 1 / 51$. arXiv: 1512.03062.

[75] C. Jacobs et al. "Finding strong lenses in CFHTLS using convolutional neural networks". In: ArXiv e-prints (Apr. 2017). arXiv: 1704 02744 [astro-ph. IM].

[76] Steven Weinberg. Gravitation and Cosmology: Principles and Applications of the General Theory of Relativity. New York, NY: Wiley, 1972. URL: https://cds. cern. ch/record/100595. 
[77] M. Bartelmann and P. Schneider. "Weak gravitational lensing". In: Phys. Rep. 340 (Jan. 2001), pp. 291-472. DOI: 10.1016/S0370-1573(00) 00082-X. eprint: astro-ph/9912508.

[78] G. Meylan et al., eds. Gravitational Lensing: Strong, Weak and Micro. 2006. eprint: astro-ph/0407232.

[79] H. Hoekstra and B. Jain. "Weak Gravitational Lensing and Its Cosmological Applications". In: Annual Review of Nuclear and Particle Science 58 (Nov. 2008), pp. 99-123. DOI: 10 . 1146/annurev . nucl . 58 . 110707 . 171151. arXiv: 0805.0139.

[80] L. van Waerbeke et al. "Magnification as a Probe of Dark Matter Halos at High Redshifts". In: The Astrophysical Journal Letters 723.1 (2010), p. L13. URL: http://stacks.iop.org/2041-8205/723/i=1/a=L13.

[81] M. Kilbinger. "Cosmology with cosmic shear observations: a review". In: Reports on Progress in Physics 78.8, 086901 (July 2015), p. 086901. DOI: 10.1088/0034-4885/78/8/086901. arXiv: 1411.0115 .

[82] M. Kilbinger et al. "Precision calculations of the cosmic shear power spectrum projection". In: ArXiv e-prints (Feb. 2017). arXiv: 1702.05301.

[83] P. Lemos, A. Challinor, and G. Efstathiou. "The effect of Limber and flat-sky approximations on galaxy weak lensing". In: ArXiv e-prints (Apr. 2017). arXiv: 1704.01054.

[84] T. D. Kitching et al. "The Limits of Cosmic Shear". In: ArXiv e-prints (Nov. 2016). arXiv: 1611.04954.

[85] R. D. Blandford et al. "Gravitational lens optics". In: Science 245 (Aug. 1989), pp. 824-830. DOI: $10.1126 /$ science.245.4920.824.

[86] M. Bartelmann. "Gravitational Lensing by Large-Scale Structures." In: Reviews in Modern Astronomy. Ed. by G. Klare. Vol. 5. Reviews in Modern Astronomy. 1992, pp. 259-270.

[87] M. Bartelmann. "Gravitational Lensing by Large-Scale Structures". In: Gravitational Lenses. Ed. by R. Kayser, T. Schramm, and L. Nieser. Vol. 406. Lecture Notes in Physics, Berlin Springer Verlag. 1992, p. 345. DOI: 10. 1007/3-540-55797-0_122.

[88] M. Bartelmann. "Cluster mass estimates from weak lensing." In: A $\& A 303$ (Nov. 1995), p. 643. eprint: astro-ph/9412051.

[89] M. Bartelmann and R. Narayan. "Gravitational lensing and the mass distribution of clusters". In: Dark Matter. Ed. by S. S. Holt and C. L. Bennett. Vol. 336. American Institute of Physics Conference Series. July 1995, pp. 307-319. DOI: 10.1063/1.48350. eprint: astro-ph/9411033.

[90] Lam Hui, Enrique Gaztañaga, and Marilena LoVerde. "Anisotropic magnification distortion of the 3D galaxy correlation. I. Real space". In: Phys. Rev. D 76 (10 Nov. 2007), p. 103502. DOI: 10.1103/PhysRevD.76.103502. URL: http://link.aps.org/doi/10.1103/PhysRevD.76.103502. 
[91] Lam Hui, Enrique Gaztañaga, and Marilena LoVerde. "Anisotropic magnification distortion of the 3D galaxy correlation. II. Fourier and redshift space". In: Phys. Rev. D 77 (6 Mar. 2008), p. 063526. DOI: 10.1103/PhysRevD. 77 .063526. URL: http://link .aps .org/doi/10 . 1103/PhysRevD . 77 . 063526.

[92] M. Bernardi et al. "Early-Type Galaxies in the Sloan Digital Sky Survey. III. The Fundamental Plane". In: AJ 125 (Apr. 2003), pp. 1866-1881. DOI: 10.1086/367794. eprint: astro-ph/0301626.

[93] Eric M. Huff and Genevieve J. Graves. "Magnificent Magnification: Exploiting the Other Half of the Lensing Signal". In: The Astrophysical Journal Letters 780.2 (2014), p. L16. URL: http://stacks . iop.org/2041$8205 / 780 / i=2 / a=L 16$.

[94] Marilena LoVerde, Lam Hui, and Enrique Gaztañaga. "Lensing corrections to features in the angular two-point correlation function and power spectrum". In: Phys. Rev. D 77 (2 Jan. 2008), p. 023512. DoI: 10.1103/ PhysRevD . 77 .023512. URL: http : / / link . aps .org/doi / 10 . 1103 / PhysRevD .77.023512.

[95] B. Ménard et al. "Measuring the galaxy-mass and galaxy-dust correlations through magnification and reddening". In: MNRAS 405 (June 2010), pp. 1025-1039. DOI: 10 . $1111 / \mathrm{j} .1365-2966$. 2010 . 16486 . x. arXiv: 0902.4240 .

[96] D. Gruen et al. "Weak lensing by galaxy troughs in DES Science Verification data". In: MNRAS 455 (Jan. 2016), pp. 3367-3380. DOI: 10.1093/mnras/ stv2506. arXiv: 1507.05090.

[97] B. Ménard et al. "Improving the accuracy of cosmic magnification statistics". In: $A \& A 403$ (June 2003), pp. 817-828. DOI: 10.1051/0004-6361: 20030406. eprint: astro-ph/0210112.

[98] J. Garcia-Bellido and T. Haugbølle. "Confronting Lemaitre Tolman Bondi models with observational cosmology". In: J. Cosmology Astropart. Phys. 4, 003 (Apr. 2008), p. 003. DOI: 10.1088/1475-7516/2008/04/003. arXiv: 0802.1523.

[99] F. Finelli et al. "Supervoids in the WISE-2MASS catalogue imprinting cold spots in the cosmic microwave background". In: MNRAS 455 (Jan. 2016), pp. 1246-1256. DOI: 10.1093/mnras/stv2388. arXiv: 1405.1555.

[100] A. Kovács and J. García-Bellido. "Cosmic troublemakers: the Cold Spot, the Eridanus supervoid, and the Great Walls". In: MNRAS 462 (Oct. 2016), pp. 1882-1893. DOI: 10.1093/mnras/stw1752. arXiv: 1511.09008.

[101] The Dark Energy Survey Collaboration. "The Dark Energy Survey". In: ArXiv Astrophysics e-prints (Oct. 2005). eprint: astro-ph/0510346. 
[102] Thomas Diehl. "The Dark Energy Survey Camera (DECam)". In: Physics Procedia 37 (2012). Proceedings of the 2nd International Conference on Technology and Instrumentation in Particle Physics (TIPP 2011), pp. 13321340. ISSN: 1875-3892. DOI: http://dx.doi.org/10.1016/j.phpro. 2012. 02 . 472. URL: http://www. sciencedirect.com/science/article/pii/ S1875389212018378.

[103] K. Batygin and M. E. Brown. "Evidence for a Distant Giant Planet in the Solar System". In: $A J$ 151, 22 (Feb. 2016), p. 22. DOI: 10.3847/00046256/151/2/22. arXiv: 1601.05438 [astro-ph.EP].

[104] E. Bertin et al. "The TERAPIX Pipeline". In: Astronomical Data Analysis Software and Systems XI. Ed. by D. A. Bohlender, D. Durand, and T. H. Handley. Vol. 281. Astronomical Society of the Pacific Conference Series. 2002, p. 228.

[105] E. Bertin and S. Arnouts. "SExtractor: Software for source extraction." In: AEAS 117 (June 1996), pp. 393-404. DOI: 10.1051/aas: 1996164.

[106] J. Zuntz et al. "IM3SHAPE: a maximum likelihood galaxy shear measurement code for cosmic gravitational lensing". In: MNRAS 434 (Sept. 2013), pp. 1604-1618. DOI: $10.1093 / \mathrm{mnras} / \mathrm{stt1125}$. arXiv: 1302.0183.

[107] E. Sheldon. NGMIX: Gaussian mixture models for $2 D$ images. Astrophysics Source Code Library. Aug. 2015. ascl: 1508.008.

[108] A. Agnello et al. "Discovery of two gravitationally lensed quasars in the Dark Energy Survey". In: MNRAS 454 (Dec. 2015), pp. 1260-1265. DOI: 10.1093/mnras/stv2171. arXiv: 1508.01203.

[109] H. Lin et al. "Discovery of the Lensed Quasar System DES J0408-5354". In: ApJ 838, L15 (Apr. 2017), p. L15. DOI: 10.3847/2041-8213/aa624e. arXiv: 1702.00072 .

[110] A. Saro et al. "Optical-SZE Scaling Relations for DES Optically Selected Clusters within the SPT-SZ Survey". In: ArXiv e-prints (May 2016). arXiv: 1605.08770.

[111] C. Sánchez et al. "Cosmic voids and void lensing in the Dark Energy Survey Science Verification data". In: MNRAS 465 (Feb. 2017), pp. 746-759. DOI: 10.1093/mnras/stw2745. arXiv: 1605.03982.

[112] L. Clerkin et al. "Testing the lognormality of the galaxy and weak lensing convergence distributions from Dark Energy Survey maps". In: MNRAS 466 (Apr. 2017), pp. 1444-1461. DOI: 10.1093/mnras/stw2106. arXiv: 1605.02036.

[113] C. Hennig et al. "Galaxy populations in massive galaxy clusters to $\mathrm{z}=$ 1.1: colour distribution, concentration, halo occupation number and red sequence fraction". In: MNRAS 467 (June 2017), pp. 4015-4035. DOI: 10.1093/mnras/stx175. arXiv: 1604.00988. 
[114] V. Vikram et al. "Wide-field lensing mass maps from Dark Energy Survey science verification data: Methodology and detailed analysis". In: Phys. Rev. D 92.2, 022006 (July 2015), p. 022006. DOI: 10.1103/PhysRevD. 92. 022006. arXiv: 1504.03002.

[115] M. R. Becker et al. "Cosmic shear measurements with Dark Energy Survey Science Verification data". In: Phys. Rev. D 94.2, 022002 (July 2016), p. 022002. DOI: 10.1103/PhysRevD.94.022002. arXiv: 1507.05598.

[116] D. Kirk et al. "Cross-correlation of gravitational lensing from DES Science Verification data with SPT and Planck lensing". In: MNRAS 459 (June 2016), pp. 21-34. DOI: 10.1093/mnras/stw570. arXiv: 1512.04535.

[117] B. Soergel et al. "Detection of the kinematic Sunyaev-Zel'dovich effect with DES Year 1 and SPT". In: MNRAS 461 (Sept. 2016), pp. 3172-3193. DOI: 10.1093/mnras/stw1455. arXiv: 1603.03904.

[118] E. Baxter et al. "Joint measurement of lensing-galaxy correlations using SPT and DES SV data". In: MNRAS 461 (Oct. 2016), pp. 4099-4114. DOI: 10.1093/mnras/stw1584. arXiv: 1602.07384.

[119] J. Prat et al. "Galaxy bias from galaxy-galaxy lensing in the DES Science Verification Data". In: ArXiv e-prints (Sept. 2016). arXiv: 1609.08167.

[120] J. Clampitt et al. "Galaxy-galaxy lensing in the Dark Energy Survey Science Verification data”. In: MNRAS 465 (Mar. 2017), pp. 4204-4218. DOI: $10.1093 / \mathrm{mnras} / \mathrm{stw2988}$. arXiv: 1603.05790.

[121] T. Giannantonio et al. "CMB lensing tomography with the DES Science Verification galaxies". In: MNRAS 456 (Mar. 2016), pp. 3213-3244. DOI: 10.1093/mnras/stv2678. arXiv: 1507.05551.

[122] A. Kovács et al. "Imprint of DES superstructures on the cosmic microwave background". In: MNRAS 465 (Mar. 2017), pp. 4166-4179. DOI: 10.1093/ mnras/stw2968. arXiv: 1610.00637.

[123] T. Abbott et al. "Cosmology from cosmic shear with Dark Energy Survey Science Verification data". In: Phys. Rev. D 94.2, 022001 (July 2016), p. 022001. DOI: 10.1103/PhysRevD.94.022001. arXiv: 1507.05552.

[124] T. Kacprzak et al. "Cosmology constraints from shear peak statistics in Dark Energy Survey Science Verification data". In: MNRAS 463 (Dec. 2016), pp. 3653-3673. DOI: 10.1093/mnras/stw2070. arXiv: 1603.05040.

[125] J. Kwan et al. "Cosmology from large-scale galaxy clustering and galaxygalaxy lensing with Dark Energy Survey Science Verification data". In: MNRAS 464 (Feb. 2017), pp. 4045-4062. DOI: 10.1093/mnras/stw2464. arXiv: 1604.07871.

[126] Dark Energy Survey Collaboration et al. "The Dark Energy Survey: more than dark energy - an overview". In: MNRAS 460 (Aug. 2016), pp. 12701299. DOI: $10.1093 / \mathrm{mnras} / \mathrm{stw641}$. arXiv: 1601.00329. 
[127] T. Broadhurst. "Mass distributions of clusters from gravitational magnification". In: Dark Matter. Ed. by S. S. Holt and C. L. Bennett. Vol. 336. American Institute of Physics Conference Series. July 1995, pp. 320-329. DOI: 10.1063/1.48351. eprint: astro-ph/9505010.

[128] A. H. Bauer et al. "Magnification of photometric LRGs by foreground LRGs and clusters in the Sloan Digital Sky Survey". In: MNRAS 440 (June 2014), pp. 3701-3713. DOI: 10.1093/mnras/stu530. arXiv: 1312.2458.

[129] J. Ford et al. "Cluster magnification and the mass-richness relation in CFHTLenS". In: MNRAS 439 (Apr. 2014), pp. 3755-3764. DOI: 10.1093/ mnras/stu225. arXiv: 1310.2295 [astro-ph.CO].

[130] I. Chiu et al. "Detection of enhancement in number densities of background galaxies due to magnification by massive galaxy clusters". In: MNRAS 457 (Apr. 2016), pp. 3050-3065. DOI: 10 . 1093 /mnras / stw190. arXiv: 1510.01745.

[131] H. Hildebrandt, L. van Waerbeke, and T. Erben. "CARS: The CFHTLSArchive-Research Survey. III. First detection of cosmic magnification in samples of normal high-z galaxies". In: $A \mathscr{E} A 507$ (Nov. 2009), pp. 683-691. DOI: $10.1051 / 0004-6361 / 200912655$. arXiv: 0906.1580.

[132] C. B. Morrison et al. "Tomographic magnification of Lyman-break galaxies in the Deep Lens Survey". In: MNRAS 426 (Nov. 2012), pp. 2489-2499. DOI: $10.1111 / j .1365-2966.2012 .21826$.x. arXiv: 1204.2830.

[133] M. Seldner and P. J. E. Peebles. "Statistical analysis of catalogs of extragalactic objects. XI - Evidence of correlation of QSOs and Lick galaxy counts". In: ApJ 227 (Jan. 1979), pp. 30-36. DOI: 10.1086/156699.

[134] C. J. Hogan, R. Narayan, and S. D. M. White. "Quasar lensing by galaxies?" In: Nature 339 (May 1989), p. 106. DOI: 10.1038/339106b0.

[135] W. Fugmann. "Statistical gravitational lensing and the Lick catalogue of galaxies". In: A\&SA 240 (Dec. 1990), pp. 11-21.

[136] M. Bartelmann and P. Schneider. "Large-scale correlations between QSOs and galaxies - an effect caused by gravitational lensing?" In: A\&̈A 268 (Feb. 1993), pp. 1-13.

[137] B. Ménard and M. Bartelmann. "Cosmological information from quasargalaxy correlations induced by weak lensing". In: A $\mathscr{G} A 386$ (May 2002), pp. 784-795. DOI: 10 . 1051/0004-6361:20020274. eprint: astro-ph / 0203163.

[138] Ryan Scranton et al. "Detection of Cosmic Magnification with the Sloan Digital Sky Survey". In: The Astrophysical Journal 633.2 (2005), p. 589. URL: http://stacks.iop.org/0004-637X/633/i=2/a=589.

[139] L. Wang et al. "HerMES: detection of cosmic magnification of submillimetre galaxies using angular cross-correlation". In: MNRAS 414 (June 2011), pp. 596-601. DOI: $10.1111 / \mathrm{j} .1365-2966.2011 .18417$ x. arXiv: 1101. 4796. 
[140] B. Jain and M. Lima. "Magnification effects on source counts and fluxes". In: $M N R A S 411$ (Mar. 2011), pp. 2113-2117. DOI: $10.1111 /$ j .13652966.2010.17505.x. arXiv: 1003.6127.

[141] C. B. Morrison and H. Hildebrandt. "Mitigating systematic errors in angular correlation function measurements from wide field surveys". In: MNRAS 454 (Dec. 2015), pp. 3121-3133. DOI: 10.1093/mnras/stv2103. arXiv: 1509.04290.

[142] H. Hildebrandt. "Observational biases in flux magnification measurements". In: MNRAS 455 (Feb. 2016), pp. 3943-3951. DOI: 10.1093/mnras/stv2575. arXiv: 1511.01352.

[143] P. Schechter. "An analytic expression for the luminosity function for galaxies." In: ApJ 203 (Jan. 1976), pp. 297-306. DOI: 10.1086/154079.

[144] R. Moessner and B. Jain. "Angular cross-correlation of galaxies - A probe of gravitational lensing by large-scale structure". In: MNRAS 294 (Feb. 1998), pp. L18-L24. DOI: 10 .1046/j . 1365-8711 . 1998.01378 .x. eprint: astro-ph/9709159.

[145] P. Fosalba et al. "The MICE Grand Challenge light-cone simulation - III. Galaxy lensing mocks from all-sky lensing maps". In: MNRAS 447 (Feb. 2015), pp. 1319-1332. DOI: 10.1093/mnras/stu2464. arXiv: 1312.2947.

[146] P. Fosalba et al. "The MICE grand challenge lightcone simulation - I. Dark matter clustering". In: MNRAS 448 (Apr. 2015), pp. 2987-3000. DOI: 10.1093/mnras/stv138. arXiv: 1312.1707.

[147] M. Crocce et al. "The MICE Grand Challenge lightcone simulation - II. Halo and galaxy catalogues". In: MNRAS 453 (Oct. 2015), pp. 1513-1530. DOI: $10.1093 / \mathrm{mnras} / \mathrm{stv} 1708$. arXiv: 1312.2013.

[148] M. Crocce et al. "Galaxy clustering, photometric redshifts and diagnosis of systematics in the DES Science Verification data". In: MNRAS 455 (Feb. 2016), pp. 4301-4324. DOI: 10.1093/mnras/stv2590. arXiv: 1507.05360.

[149] C. Chang et al. "Modeling the Transfer Function for the Dark Energy Survey". In: The Astrophysical Journal 801.2 (2015), p. 73. URL: http: //stacks. iop.org/0004-637X/801/i=2/a=73.

[150] R. Mandelbaum et al. "Systematic errors in weak lensing: application to SDSS galaxy-galaxy weak lensing". In: MNRAS 361 (Aug. 2005), pp. 12871322. DOI: $10.1111 / \mathrm{j} .1365-2966.2005$. 09282 .x. eprint: astro-ph / 0501201.

[151] I. Sevilla et al. "The Dark Energy Survey Data Management System". In: ArXiv e-prints (Sept. 2011). arXiv: 1109.6741 [astro-ph. IM] .

[152] S. Desai et al. "The Blanco Cosmology Survey: Data Acquisition, Processing, Calibration, Quality Diagnostics, and Data Release". In: ApJ 757, 83 (Sept. 2012), p. 83. DOI: $10.1088 / 0004-637 \mathrm{X} / 757 / 1 / 83$. arXiv: 1204.1210 [astro-ph.CO]. 
[153] J. J. Mohr et al. "The Dark Energy Survey data processing and calibration system". In: Software and Cyberinfrastructure for Astronomy II. Vol. 8451. Proc. SPIE. Sept. 2012, p. 84510D. DOI: 10 . $1117 / 12$.926785. arXiv: 1207.3189 [astro-ph.IM].

[154] M. E. C. Swanson et al. "Methods for rapidly processing angular masks of next-generation galaxy surveys". In: MNRAS 387 (July 2008), pp. 13911402. DOI: $10.1111 / \mathrm{j} .1365-2966.2008 .13296 . x$. arXiv: 0711.4352.

[155] K. M. Górski et al. "HEALPix: A Framework for High-Resolution Discretization and Fast Analysis of Data Distributed on the Sphere". In: ApJ 622 (Apr. 2005), pp. 759-771. DOI: 10.1086/427976. eprint: astro-ph/0409513.

[156] M. Carrasco Kind and R. J. Brunner. "TPZ: photometric redshift PDFs and ancillary information by using prediction trees and random forests". In: MNRAS 432 (June 2013), pp. 1483-1501. DOI: 10.1093/mnras/stt574. arXiv: 1303.7269.

[157] C. Sánchez et al. "Photometric redshift analysis in the Dark Energy Survey Science Verification data". In: MNRAS 445 (Dec. 2014), pp. 1482-1506. DOI: $10.1093 / \mathrm{mnras} / \mathrm{stu} 1836$. arXiv: 1406.4407 [astro-ph.IM] .

[158] M. Jarvis, G. Bernstein, and B. Jain. "The skewness of the aperture mass statistic". In: MNRAS 352 (July 2004), pp. 338-352. DOI: 10.1111/j.13652966.2004.07926.x. eprint: astro-ph/0307393.

[159] S. D. Landy and A. S. Szalay. "Bias and variance of angular correlation functions". In: ApJ 412 (July 1993), pp. 64-71. DOI: 10.1086/172900.

[160] E. Suchyta et al. "No galaxy left behind: accurate measurements with the faintest objects in the Dark Energy Survey". In: MNRAS 457 (Mar. 2016), pp. 786-808. DOI: 10.1093/mnras/stv2953. arXiv: 1507.08336.

[161] James MacQueen et al. "Some methods for classification and analysis of multivariate observations". In: Proceedings of the fifth Berkeley symposium on mathematical statistics and probability. Vol. 1. 14. Oakland, CA, USA. 1967, pp. 281-297.

[162] F. Pedregosa et al. "Scikit-learn: Machine Learning in Python". In: Journal of Machine Learning Research 12 (2011), pp. 2825-2830.

[163] Planck Collaboration. "Planck 2015 results. XIII. Cosmological parameters". In: A\&A 594, A13 (Sept. 2016), A13. DOI: 10.1051/0004-6361/201525830. arXiv: 1502.01589.

[164] C. Chang et al. "Galaxy bias from the Dark Energy Survey Science Verification data: combining galaxy density maps and weak lensing maps". In: MNRAS 459 (July 2016), pp. 3203-3216. DOI: 10.1093/mnras/stw861. arXiv: 1601.00405.

[165] Robert E. Kass and Adrian E. Raftery. "Bayes Factors". In: Journal of the American Statistical Association 90.430 (1995), pp. 773-795. ISSN: 01621459. URL: http://www. jstor.org/stable/2291091. 
[166] A. J. Ross et al. "The clustering of galaxies in the SDSS-III Baryon Oscillation Spectroscopic Survey: analysis of potential systematics". In: MNRAS 424 (July 2012), pp. 564-590. DOI: $10.1111 /$ j.1365-2966.2012.21235.x. arXiv: 1203.6499.

[167] B. Leistedt et al. "Mapping and simulating systematics due to spatiallyvarying observing conditions in DES Science Verification data". In: ArXiv e-prints (July 2015). arXiv: 1507.05647.

[168] S. Ho et al. "Clustering of Sloan Digital Sky Survey III Photometric Luminous Galaxies: The Measurement, Systematics, and Cosmological Implications". In: ApJ 761, 14 (Dec. 2012), p. 14. DOI: $10.1088 / 0004-$ 637X/761/1/14. arXiv: 1201.2137.

[169] F. Elsner, B. Leistedt, and H. V. Peiris. "Unbiased methods for removing systematics from galaxy clustering measurements". In: MNRAS 456 (Feb. 2016), pp. 2095-2104. DOI: 10.1093/mnras/stv2777. arXiv: 1509.08933.

[170] C. Bonnett et al. "Redshift distributions of galaxies in the Dark Energy Survey Science Verification shear catalogue and implications for weak lensing". In: Phys. Rev. D 94 (4 Aug. 2016), p. 042005. DOI: 10.1103/ PhysRevD . 94 .042005. URL: http : / / link . aps . org/doi / 10 . 1103 / PhysRevD .94.042005.

[171] G. Bernstein and D. Huterer. "Catastrophic photometric redshift errors: weak-lensing survey requirements". In: MNRAS 401 (Jan. 2010), pp. 13991408. DOI: $10.1111 / \mathrm{j} .1365-2966$. 2009 .15748 x. arXiv: 0902.2782 [astro-ph.CO].

[172] J. Asorey et al. "Galaxy clustering with photometric surveys using PDF redshift information". In: MNRAS 459 (June 2016), pp. 1293-1309. DOI: 10.1093/mnras/stw721. arXiv: 1601.00357.

[173] J. A. Newman. "Calibrating Redshift Distributions beyond Spectroscopic Limits with Cross-Correlations". In: ApJ 684, 88-101 (Sept. 2008), pp. 88101. DOI: $10.1086 / 589982$. arXiv: 0805.1409.

[174] D. J. Matthews and J. A. Newman. "Reconstructing Redshift Distributions with Cross-correlations: Tests and an Optimized Recipe". In: ApJ 721 (Sept. 2010), pp. 456-468. DOI: 10.1088/0004-637X/721/1/456. arXiv: 1003.0687 [astro-ph.CO].

[175] B. Ménard et al. "Clustering-based redshift estimation: method and application to data". In: ArXiv e-prints (Mar. 2013). arXiv: 1303.4722.

[176] V. Scottez et al. "Clustering-based redshift estimation: application to VIPERS/CFHTLS". In: MNRAS 462 (Oct. 2016), pp. 1683-1696. DOI: 10.1093/mnras/stw1500. arXiv: 1605.05501.

[177] P. Graff et al. "SKYNET: an efficient and robust neural network training tool for machine learning in astronomy". In: MNRAS 441 (June 2014), pp. 17411759. DOI: $10.1093 / \mathrm{mnras} / \mathrm{stu} 642$. arXiv: 1309.0790 [astro-ph.IM].

[178] N. Benítez. "Bayesian Photometric Redshift Estimation". In: ApJ 536 (June 2000), pp. 571-583. DOI: 10.1086/308947. eprint: astro-ph/9811189. 
[179] P. Melchior et al. "First measurement of gravitational lensing by cosmic voids in SDSS". In: MNRAS 440 (June 2014), pp. 2922-2927. DOI: 10 . 1093/mnras/stu456. arXiv: 1309.2045.

[180] E. S. Rykoff et al. "redMaPPer. I. Algorithm and SDSS DR8 Catalog". In: ApJ 785, 104 (Apr. 2014), p. 104. DOI: 10.1088/0004-637X/785/2/104. arXiv: 1303.3562.

[181] E. Rozo et al. "redMaGiC: selecting luminous red galaxies from the DES Science Verification data". In: MNRAS 461 (Sept. 2016), pp. 1431-1450. DOI: $10.1093 / \mathrm{mnras} / \mathrm{stw1281}$. arXiv: 1507.05460 [astro-ph. IM] .

[182] E. S. Rykoff et al. "The RedMaPPer Galaxy Cluster Catalog From DES Science Verification Data". In: ApJS 224, 1 (May 2016), p. 1. DOI: 10.3847/ 0067-0049/224/1/1. arXiv: 1601.00621.

[183] O. Friedrich and T. Eifler. "Precision matrix expansion - efficient use of numerical simulations in estimating errors on cosmological parameters". In: ArXiv e-prints (Mar. 2017). arXiv: 1703.07786 [astro-ph.IM]. 




$+x^{2}+\frac{\pi}{D}$

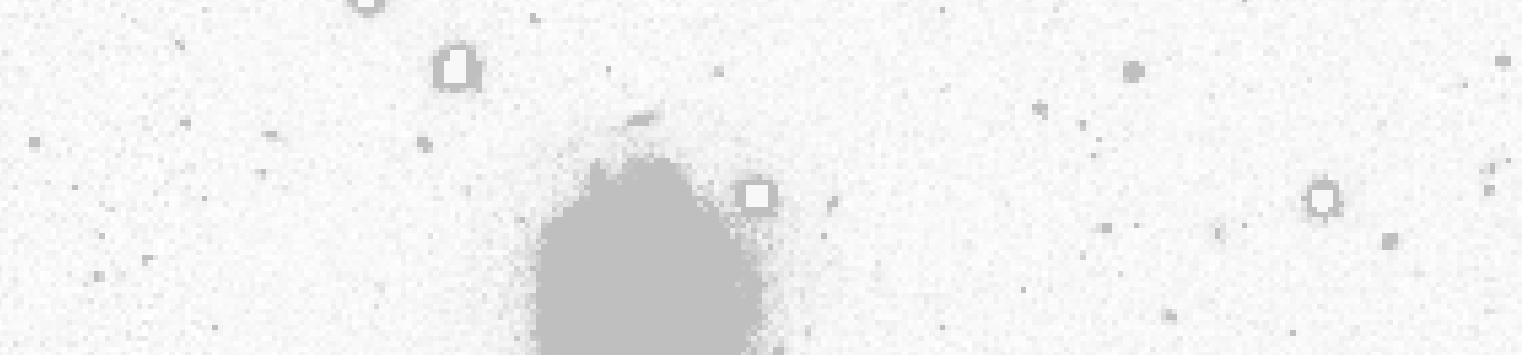

thr

ㄴ.7.

ㄴ.

C IF

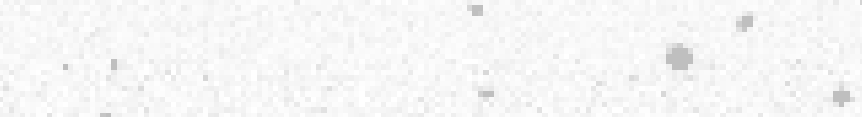

$y=$

this

$=$

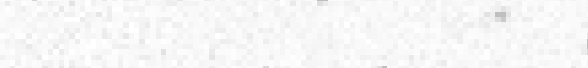

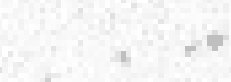

4

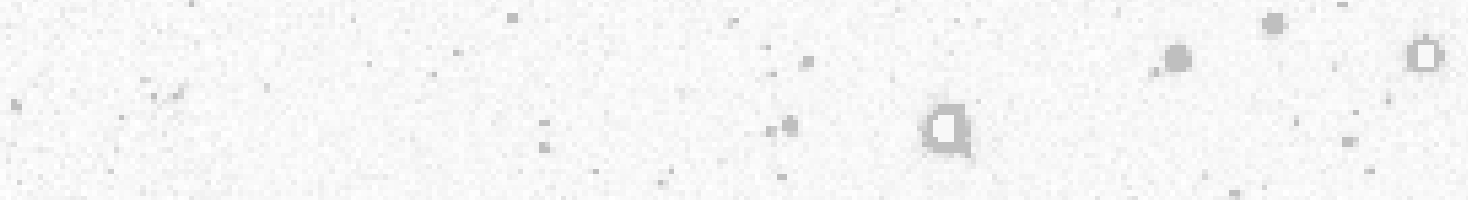

at

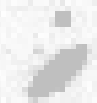
$+$ th in
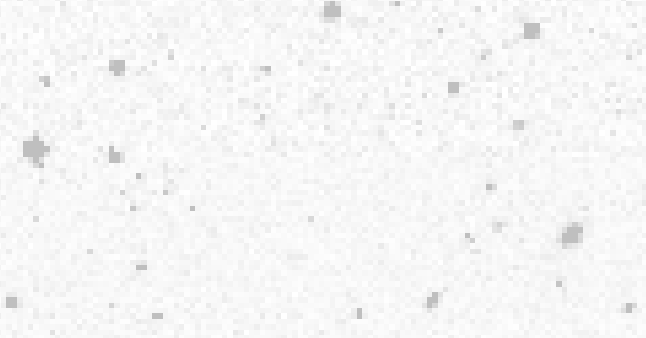

푼

it

$+$

$+$ 